\title{
Chiral EFT based nuclear forces: Achievements and challenges
}

\author{
R Machleidt and F Sammarruca \\ Department of Physics, University of Idaho, Moscow, Idaho 83844, U.S.A. \\ E-mail: machleid@uidaho.edu, fsammarr@uidaho.edu
}

\begin{abstract}
During the past two decades, chiral effective field theory has become a popular tool to derive nuclear forces from first principles. Two-nucleon interactions have been worked out up to sixth order of chiral perturbation theory and three-nucleon forces up to fifth order. Applications of some of these forces have been conducted in nuclear few- and many-body systems - with a certain degree of success. But in spite of these achievements, we are still faced with great challenges. Among them is the issue of a proper uncertainty quantification of predictions obtained when applying these forces in $a b$ initio calculations of nuclear structure and reactions. A related problem is the order by order convergence of the chiral expansion. We start this review with a pedagogical introduction and then present the current status of the field of chiral nuclear forces. This is followed by a discussion of representative examples for the application of chiral two- and three-body forces in the nuclear many-body system including convergence issues.
\end{abstract}

PACS numbers: 13.75.Cs, 12.39.Fe, 21.30.-x, 21.45.-v, 21.65.-f

Submitted to: Phys. Scr. 


\section{Historical Perspective}

In 1975, when A. Bohr, B. Mottelson, and L. Rainwater were honored with the Nobel Prize, the Reid potential [1] was the most popular nucleon-nucleon $(N N)$ potential within the international nuclear physics community. It was applied in most miscroscopic nuclear structure calculations produced in the 1970's. The Reid potential is a phenomenological potential that was considered very quantitative by the standards of the time and easy to use, which explains its popularity. However, attempts to derive the $N N$ interaction on fundamental grounds had been around for quite a while. Since the mid 1960's, one-boson exchange potentials were being developed [2], which by the mid 1970's assumed a quantitative character comparable to the Reid potential [3, 4, 5]. Moreover, research that went beyond the simple one-boson-exchange assumption (which always includes a 'fictitious' $\sigma$-boson) was also under way. The most notable work of this kind became known as the Paris [6] and the Bonn potentials [7].

Since the more sophisticated meson models seemed to have a sound theoretical foundation and, in addition, were quantitatively very successful, it appeared that they were the solution of the nuclear force problem. However, with the discovery (in the 1970 's) that the fundamental theory of strong interactions is quantum chromodynamics (QCD) and not meson theory, all "meson theories" had to be viewed as models, and the attempts to derive the nuclear force from first principals had to start all over again.

The problem with a derivation of nuclear forces from QCD is two-fold. First, each nucleon consists of three valence quarks, quark-antiquark pairs, and gluons such that the system of two nucleons is a complicated many-body problem. Second, the force between quarks, which is created by the exchange of gluons, has the feature of being very strong at the low energy-scale that is characteristic of nuclear physics. This extraordinary strength makes it difficult to find converging expansions. Therefore, during the first round of new attempts, QCD-inspired quark models became popular. The positive aspect of these models is that they try to explain nucleon structure (which consists of three quarks) and nucleon-nucleon interactions (six-quark systems) on an equal footing. Some of the gross features of the two-nucleon force, like the "hard core", are explained successfully in such models. However, from a critical point of view, it must be noted that these quark-based approaches are yet another set of models and not a theory. Alternatively, one may try to solve the six-quark problem with brute computing power, by putting the six-quark system on a four dimensional lattice of discrete points which represents the three dimensions of space and one dimension of time. This method has become known as lattice QCD and is making progress. However, such calculations are computationally very expensive and cannot be used as a standard nuclear physics tool.

Around 1980, a major breakthrough occurred when the nobel laureate Steven Weinberg applied the concept of an effective field theory (EFT) to low-energy QCD [8, 9]. He simply wrote down the most general Lagrangian that is consistent with all the properties of low-energy QCD, since that would make this theory equivalent to lowenergy QCD. A particularly important property is $S U(2)_{R} \times S U(2)_{L}$ symmetry, the 
so-called chiral symmetry, which is "spontaneously" broken. Massless spin- $\frac{1}{2}$ fermions posses chirality, which means that their spin and momentum are either parallel to each other ("right-handed") or anti-parallel ("left-handed") and remain so forever. Since the quarks, which nucleons are made of ("up" and "down" quarks), are almost massless, approximate chiral symmetry is a given. Naively, this symmetry should have the consequence that one finds in nature mesons of the same mass, but with positive and negative parity. However, this is not the case and such failure is termed a "spontaneous" breaking of the symmetry. According to a theorem first proven by Goldstone, the spontaneous breaking of a symmetry implies the existence of a particle, here, the pseudoscalar pion. Thus, the pion becomes the main player in generating the nuclear force. The interaction of pions with nucleons is weak at low energies as compared to the interaction of gluons with quarks. Therefore, pion-nucleon processes can be calculated without problem. Moreover, this effective field theory can be expanded in powers of momentum over "scale", where scale denotes the "chiral symmetry breaking scale" which is about $1 \mathrm{GeV}$. This scheme is also known as chiral perturbation theory (ChPT) and allows to calculate the various terms that make up the nuclear potential systematically power by power, or order by order. Another advantage of the chiral EFT approach is its ability to generate not only the force between two nucleons, but also many-nucleon forces, on the same footing [10]. In modern theoretical nuclear physics, the chiral EFT approach is becoming increasingly popular and is applied with great success [11, 12, 13, 14, 15].

This article is organized as follows. In Sec. 2, we will present a pedagogical introduction into the EFT approach to low-energy QCD, including the development of effective Lagrangians. Section 3 provides a broad overview of the hierarchy of nuclear forces as they emerge from EFT. Sections 4 to 6 then spell out in detail the development of the two-nucleon forces from long-range to short-range and the construction of quantitative $N N$ potentials. Chiral many-body forces are presented in Sec. 7. Applications of chiral forces in the many-body problem and convergence issues are discussed in Sec. 8, and Sec. 9 concludes the article.

\section{Effective field theory for low-energy QCD}

Quantum chromodynamics provides the theoretical framework to describe strong interactions, namely interactions involving quarks and gluons. According to QCD, objects which carry color interact weakly at short distances and strongly at large distances, where the separation between the two regimes is about $1 \mathrm{fm}$. Naturally, short distances and long distances can be associated with high and low energies, respectively, causing the quarks to be confined into hadrons, which carry no color. At the same time, the weak nature of the force at high energies results into what is known as "asymptotic freedom". (We note that these behaviors originate from the fact that QCD is a non-Abelian gauge field theory with color $S U(3)$ the underlying gauge group.) Therefore, QCD is perturbative at high energy, but strongly coupled at low-energy. The 
Table 1. Abbreviations and acronyms used in this article.

\begin{tabular}{|c|c|}
\hline Abbreviation/Acronym & Explanation \\
\hline CCWZ & Callan, Coleman, Wess, and Zumino 20 \\
\hline ChPT & chiral perturbation theory \\
\hline CMS & center-of-mass system \\
\hline ct & contact (term) \\
\hline EFT & effective field theory \\
\hline EoS & equation of state \\
\hline FFG & free Fermi gas \\
\hline GW & George Washington (University) \\
\hline HI & heavy ion \\
\hline IANM & isospin-asymmetric nuclear matter \\
\hline $\mathrm{KH}$ & Karlsruhe (University) \\
\hline LEC & low-energy constant \\
\hline $\mathrm{LO}$ & leading order \\
\hline $\mathrm{LS}$ & Lippmann-Schwinger \\
\hline $\mathrm{NLO}$ & next-to-leading order \\
\hline NM & neutron matter \\
\hline$N N$ & nucleon-nucleon \\
\hline NNLO, $\mathrm{N}^{2} \mathrm{LO}$ & next-to-next-to-leading order \\
\hline $\mathrm{N}^{3} \mathrm{LO}, \ldots$ & next-to-next-to-next-to-leading order, ... \\
\hline PREX & lead radius experiment \\
\hline PWA & partial-wave analysis \\
\hline QCD & quantum chromodynamics \\
\hline SFR & spectral function renormalization 38 \\
\hline SNM & symmetric nuclear matter \\
\hline$S U(n)$ & special unitary group in $n$ dimensions \\
\hline VPI & Virginia Polytechnic Institute \\
\hline $1 \mathrm{PE}$ & one-pion exchange \\
\hline $2 \mathrm{PE}$ & two-pion exchange \\
\hline $3 \mathrm{PE}$ & three-pion exchange \\
\hline $2 \mathrm{NF}$ & two-nucleon force \\
\hline $3 \mathrm{NF}$ & three-nucleon force \\
\hline $4 \mathrm{NF}$ & four-nucleon force \\
\hline
\end{tabular}

energies typical for nuclear physics are low and, thus, nucleons are appropriate degrees of freedom. The nuclear force can then be regarded as a residual color interaction acting between nucleons in a way similar to how the van der Waals forces bind neutral molecules. If described in terms of quark and gluon degrees of freedom, the interaction between nucleons is an extremely complex problem, which can be confronted with the computational methods known as lattice QCD. In a recent paper [16], the nucleonnucleon system is investigated at a pion mass of about $450 \mathrm{MeV}$. Over the range of energies that are studied, the scattering phase shifts in the ${ }^{1} S_{0}$ and ${ }^{3} S-D_{1}$ channels are found to be similar to those in nature and indicate a repulsive short-range component of the interaction. This result is then extrapolated to the physical pion mass with the help of chiral perturbation theory. The pion mass of $450 \mathrm{MeV}$ is still too large to allow 
for reliable extrapolations, but the feasibility has been demonstrated and more progress can be expected for the near future. In a lattice calculation of a very different kind, the nucleon-nucleon $(N N)$ potential was studied in Ref. [17]. The central component of this potential exhibits repulsion at the core as well as intermediate-range attraction. This is encouraging, but one must keep in mind that the pion masses employed in this study are still quite large. In summary, although calculations within lattice QCD are being performed and improved, they are computationally very costly, and thus they are useful, in practice, only to explore a few cases. Clearly, a different approach is necessary to address a full variety of nuclear structure problems.

The concept of an effective field theory shows an alternative and realistic way to proceed. The first step towards the development of an EFT is the identification of appropriate scales. The large difference between the masses of the pions and the masses of the vector mesons, like $\rho(770)$ and $\omega(782)$, provides a clue. From that observation, one is prompted to take the pion mass as the identifier of the soft scale, $Q \sim m_{\pi}$, while the rho mass sets the hard scale, $\Lambda_{\chi} \sim m_{\rho}$, often referred to as the chiral-symmetry breaking scale. It is then natural to consider an expansion in terms of $Q / \Lambda_{\chi}$. With regard to the choice of degrees of freedom, we observed earlier that, as far as conventional nuclear physics is concerned, quarks and gluons are ineffective and thus nucleons and pions should be taken as the appropriate degrees of freedom. Of course, we do not wish to construct yet one more phenomenological model and, therefore, our EFT must be firmly linked with QCD. This strong link is present if we require the EFT to be consistent with the symmetries of QCD. The meaning and relevance of such statement is expressed in the so-called 'folk theorem' by Weinberg [8]:

If one writes down the most general possible Lagrangian, including all terms consistent with assumed symmetry principles, and then calculates matrix elements with this Lagrangian to any given order of perturbation theory, the result will simply be the most general possible S-matrix consistent with analyticity, perturbative unitarity, cluster decomposition, and the assumed symmetry principles.

To summarize, the development of a proper EFT must proceed as follows:

(i) Identify the low- and high-energy scales, and the degrees of freedom suitable for (low-energy) nuclear physics.

(ii) Recognize the symmetries of low-energy QCD and explore the mechanisms responsible of their breakings.

(iii) Build the most general Lagrangian which respects those (broken) symmetries.

(iv) Formulate a scheme to organize contributions in order of their importance. Clearly, this amounts to performing an expansion in terms of (low) momenta.

(v) Using the expansion mentioned above, evaluate Feynman diagrams to any desired accuracy.

In what follows, we will discuss each of the steps above. Note that the first one has already been addressed, so we will move directly to the second one. 


\subsection{Symmetries of low-energy $Q C D$}

Our purpose here is to provide a compact introduction into (low-energy) QCD, with particular attention to the symmetries and their breakings. For more details the reader is referred to Refs. [11, 18].

2.1.1. Chiral symmetry We begin with the QCD Lagrangian,

$$
\mathcal{L}_{\mathrm{QCD}}=\bar{q}\left(i \gamma^{\mu} \mathcal{D}_{\mu}-\mathcal{M}\right) q-\frac{1}{4} \mathcal{G}_{\mu \nu, a} \mathcal{G}_{a}^{\mu \nu}
$$

with the gauge-covariant derivative

$$
\mathcal{D}_{\mu}=\partial_{\mu}-i g \frac{\lambda_{a}}{2} \mathcal{A}_{\mu, a}
$$

and the gluon field strength tensorf

$$
\mathcal{G}_{\mu \nu, a}=\partial_{\mu} \mathcal{A}_{\nu, a}-\partial_{\nu} \mathcal{A}_{\mu, a}+g f_{a b c} \mathcal{A}_{\mu, b} \mathcal{A}_{\nu, c}
$$

In the above, $q$ denotes the quark fields and $\mathcal{M}$ the quark mass matrix. Further, $g$ is the strong coupling constant and $\mathcal{A}_{\mu, a}$ are the gluon fields. Moreover, $\lambda_{a}$ are the Gell-Mann matrices and $f_{a b c}$ the structure constants of the $S U(3)$ color Lie algebra $(a, b, c=1, \ldots, 8)$; summation over repeated indices is always implied. The gluon-gluon term in the last equation arises from the non-Abelian nature of the gauge theory and is the reason for the peculiar features of the color force.

The current masses of the up $(u)$, down $(d)$, and strange (s) quarks are in a $\overline{M S}$ scheme at a scale of $\mu \approx 2 \mathrm{GeV}[19]$ :

$$
\begin{aligned}
& m_{u}=2.3 \pm 0.7 \mathrm{MeV} \\
& m_{d}=4.8 \pm 0.5 \mathrm{MeV} \\
& m_{s}=95 \pm 5 \mathrm{MeV}
\end{aligned}
$$

These masses are small as compared to a typical hadronic scale such as the mass of a light hadron other than a Goldstone bosons, e.g., $m_{\rho}=0.78 \mathrm{GeV} \approx 1 \mathrm{GeV}$.

Thus it is relevant to discuss the QCD Lagrangian in the case when the quark masses vanish:

$$
\mathcal{L}_{\mathrm{QCD}}^{0}=\bar{q} i \gamma^{\mu} \mathcal{D}_{\mu} q-\frac{1}{4} \mathcal{G}_{\mu \nu, a} \mathcal{G}_{a}^{\mu \nu}
$$

Right- and left-handed quark fields are defined as

$$
q_{R}=P_{R} q, \quad q_{L}=P_{L} q
$$

with

$$
P_{R}=\frac{1}{2}\left(1+\gamma_{5}\right), \quad P_{L}=\frac{1}{2}\left(1-\gamma_{5}\right)
$$

Then the Lagrangian can be rewritten as

$$
\mathcal{L}_{\mathrm{QCD}}^{0}=\bar{q}_{R} i \gamma^{\mu} \mathcal{D}_{\mu} q_{R}+\bar{q}_{L} i \gamma^{\mu} \mathcal{D}_{\mu} q_{L}-\frac{1}{4} \mathcal{G}_{\mu \nu, a} \mathcal{G}_{a}^{\mu \nu}
$$

$\ddagger$ For $S U(N)$ group indices, we use Latin letters, $\ldots, a, b, c, \ldots, i, j, k, \ldots$, and, in general, do not distinguish between subscripts and superscripts. 
This equation revels that the right- and left-handed components of massless quarks do not mix in the QCD Lagrangian. For the two-flavor case, this is $S U(2)_{R} \times S U(2)_{L}$ symmetry, also known as chiral symmetry. However, this symmetry is broken in two ways: explicitly and spontaneously.

2.1.2. Explicit symmetry breaking The mass term $-\bar{q} \mathcal{M} q$ in the QCD Lagrangian Eq. (1) breaks chiral symmetry explicitly. To better see this, let's rewrite $\mathcal{M}$ for the two-flavor case,

$$
\begin{aligned}
\mathcal{M} & =\left(\begin{array}{cc}
m_{u} & 0 \\
0 & m_{d}
\end{array}\right) \\
& =\frac{1}{2}\left(m_{u}+m_{d}\right)\left(\begin{array}{ll}
1 & 0 \\
0 & 1
\end{array}\right)+\frac{1}{2}\left(m_{u}-m_{d}\right)\left(\begin{array}{cc}
1 & 0 \\
0 & -1
\end{array}\right) \\
& =\frac{1}{2}\left(m_{u}+m_{d}\right) I+\frac{1}{2}\left(m_{u}-m_{d}\right) \tau_{3} .
\end{aligned}
$$

The first term in the last equation in invariant under $S U(2)_{V}$ (isospin symmetry) and the second term vanishes for $m_{u}=m_{d}$. Therefore, isospin is an exact symmetry if $m_{u}=m_{d}$. However, both terms in Eq. (11) break chiral symmetry. Since the up and down quark masses [Eqs. (4) and (5)] are small as compared to the typical hadronic mass scale of $\sim 1 \mathrm{GeV}$, the explicit chiral symmetry breaking due to non-vanishing quark masses is very small.

2.1.3. Spontaneous symmetry breaking A (continuous) symmetry is said to be spontaneously broken if a symmetry of the Lagrangian is not realized in the ground state of the system. There is evidence that the (approximate) chiral symmetry of the QCD Lagrangian is spontaneously broken - for dynamical reasons of nonperturbative origin which are not fully understood at this time. The most plausible evidence comes from the hadron spectrum.

From chiral symmetry, one naively expects the existence of degenerate hadron multiplets of opposite parity, i.e., for any hadron of positive parity one would expect a degenerate hadron state of negative parity and vice versa. However, these "parity doublets" are not observed in nature. For example, take the $\rho$-meson which is a vector meson of negative parity $\left(J^{P}=1^{-}\right)$and mass $776 \mathrm{MeV}$. There does exist a $1^{+}$meson, the $a_{1}$, but it has a mass of $1230 \mathrm{MeV}$ and, therefore, cannot be perceived as degenerate with the $\rho$. On the other hand, the $\rho$ meson comes in three charge states (equivalent to three isospin states), the $\rho^{ \pm}$and the $\rho^{0}$, with masses that differ by at most a few $\mathrm{MeV}$. Thus, in the hadron spectrum, $S U(2)_{V}$ (isospin) symmetry is well observed, while axial symmetry is broken: $S U(2)_{R} \times S U(2)_{L}$ is broken down to $S U(2)_{V}$.

A spontaneously broken global symmetry implies the existence of (massless) Goldstone bosons. The Goldstone bosons are identified with the isospin triplet of the (pseudoscalar) pions, which explains why pions are so light. The pion masses are not exactly zero because the up and down quark masses are not exactly zero either 
(explicit symmetry breaking). Thus, pions are a truly remarkable species: they reflect spontaneous as well as explicit symmetry breaking. Goldstone bosons interact weakly at low energy. They are degenerate with the vacuum and, therefore, interactions between them must vanish at zero momentum and in the chiral limit $\left(m_{\pi} \rightarrow 0\right)$.

\subsection{Chiral effective Lagrangians}

The next step in our EFT program is to build the most general Lagrangian consistent with the (broken) symmetries discussed above. An elegant formalism for the construction of such Lagrangians was developed by Callan, Coleman, Wess, and Zumino (CCWZ) [20] who developed the foundations of non-linear realizations of chiral symmetry from the point of view of group theory $\{$ The Lagrangians we give below are built upon the CCWZ formalism.

We already addressed the fact that the appropriate degrees of freedom are pions (Goldstone bosons) and nucleons. Because pion interactions must vanish at zero momentum transfer and in the limit of $m_{\pi} \rightarrow 0$, namely the chiral limit, the Lagrangian is expanded in powers of derivatives and pion masses. More precisely, the Lagrangian is expanded in powers of $Q / \Lambda_{\chi}$ where $Q$ stands for a (small) momentum or pion mass and $\Lambda_{\chi} \approx 1 \mathrm{GeV}$ is identified with the hard scale. These are the basic steps behind the chiral perturbative expansion.

Schematically, we can write the effective Lagrangian as

$$
\mathcal{L}=\mathcal{L}_{\pi \pi}+\mathcal{L}_{\pi N}+\mathcal{L}_{N N}+\ldots,
$$

where $\mathcal{L}_{\pi \pi}$ deals with the dynamics among pions, $\mathcal{L}_{\pi N}$ describes the interaction between pions and a nucleon, and $\mathcal{L}_{N N}$ contains two-nucleon contact interactions which consist of four nucleon-fields (four nucleon legs) and no meson fields. The ellipsis stands for terms that involve two nucleons plus pions and three or more nucleons with or without pions, relevant for nuclear many-body forces (an example for this in lowest order are the last two terms of Eq. (18), below). The individual Lagrangians are organized order by order:

$$
\begin{aligned}
& \mathcal{L}_{\pi \pi}=\mathcal{L}_{\pi \pi}^{(2)}+\mathcal{L}_{\pi \pi}^{(4)}+\ldots, \\
& \mathcal{L}_{\pi N}=\mathcal{L}_{\pi N}^{(1)}+\mathcal{L}_{\pi N}^{(2)}+\mathcal{L}_{\pi N}^{(3)}+\mathcal{L}_{\pi N}^{(4)}+\mathcal{L}_{\pi N}^{(5)}+\ldots,
\end{aligned}
$$

and

$$
\mathcal{L}_{N N}=\mathcal{L}_{N N}^{(0)}+\mathcal{L}_{N N}^{(2)}+\mathcal{L}_{N N}^{(4)}+\mathcal{L}_{N N}^{(6)}+\ldots
$$

where the superscript refers to the number of derivatives or pion mass insertions (chiral dimension) and the ellipsis stands for terms of higher dimensions.

Above, we have organized the Lagrangians by the number of derivatives or pionmasses. This is the standard way, appropriate particularly for considerations of $\pi-\pi$ $\S$ An accessible introduction into the rather involved CCWZ formalism can be found in Ref. [18]. 
and $\pi-N$ scattering. As it turns out (cf. Section 3.1), for interactions among nucleons, sometimes one makes use of the so-called index of the interaction,

$$
\Delta \equiv d+\frac{n}{2}-2
$$

where $d$ is the number of derivatives or pion-mass insertions and $n$ the number of nucleon field operators (nucleon legs). We will now write down the Lagrangian in terms of increasing values of the parameter $\Delta$ and we will do so using the so-called heavy-baryon formalism which we indicate by a "hat" [21].

The leading-order Lagrangian reads,

$$
\begin{aligned}
\widehat{\mathcal{L}}^{\Delta=0}= & \frac{1}{2} \partial_{\mu} \boldsymbol{\pi} \cdot \partial^{\mu} \boldsymbol{\pi}-\frac{1}{2} m_{\pi}^{2} \boldsymbol{\pi}^{2} \\
& +\frac{1-4 \alpha}{2 f_{\pi}^{2}}\left(\boldsymbol{\pi} \cdot \partial_{\mu} \boldsymbol{\pi}\right)\left(\boldsymbol{\pi} \cdot \partial^{\mu} \boldsymbol{\pi}\right)-\frac{\alpha}{f_{\pi}^{2}} \boldsymbol{\pi}^{2} \partial_{\mu} \boldsymbol{\pi} \cdot \partial^{\mu} \boldsymbol{\pi}+\frac{8 \alpha-1}{8 f_{\pi}^{2}} m_{\pi}^{2} \boldsymbol{\pi}^{4} \\
& +\bar{N}\left[i \partial_{0}-\frac{g_{A}}{2 f_{\pi}} \boldsymbol{\tau} \cdot(\vec{\sigma} \cdot \vec{\nabla}) \boldsymbol{\pi}-\frac{1}{4 f_{\pi}^{2}} \boldsymbol{\tau} \cdot\left(\boldsymbol{\pi} \times \partial_{0} \boldsymbol{\pi}\right)\right] N \\
& +\bar{N}\left\{\frac{g_{A}(4 \alpha-1)}{4 f_{\pi}^{3}}(\boldsymbol{\tau} \cdot \boldsymbol{\pi})[\boldsymbol{\pi} \cdot(\vec{\sigma} \cdot \vec{\nabla}) \boldsymbol{\pi}]\right. \\
& \left.+\frac{g_{A} \alpha}{2 f_{\pi}^{3}} \boldsymbol{\pi}^{2}[\boldsymbol{\tau} \cdot(\vec{\sigma} \cdot \vec{\nabla}) \boldsymbol{\pi}]\right\} N \\
& -\frac{1}{2} C_{S} \bar{N} N \bar{N} N-\frac{1}{2} C_{T}(\bar{N} \vec{\sigma} N) \cdot(\bar{N} \vec{\sigma} N)+\ldots
\end{aligned}
$$

and subleading Lagrangians are,

$$
\begin{aligned}
\widehat{\mathcal{L}}^{\Delta=1}= & \bar{N}\left\{\frac{\vec{\nabla}^{2}}{2 M_{N}}-\frac{i g_{A}}{4 M_{N} f_{\pi}} \boldsymbol{\tau} \cdot\left[\vec{\sigma} \cdot\left(\overleftarrow{\nabla} \partial_{0} \boldsymbol{\pi}-\partial_{0} \boldsymbol{\pi} \vec{\nabla}\right)\right]\right. \\
& \left.-\frac{i}{8 M_{N} f_{\pi}^{2}} \boldsymbol{\tau} \cdot[\overleftarrow{\nabla} \cdot(\boldsymbol{\pi} \times \vec{\nabla} \boldsymbol{\pi})-(\boldsymbol{\pi} \times \vec{\nabla} \boldsymbol{\pi}) \cdot \vec{\nabla}]\right\} N \\
& +\bar{N}\left[4 c_{1} m_{\pi}^{2}-\frac{2 c_{1}}{f_{\pi}^{2}} m_{\pi}^{2} \boldsymbol{\pi}^{2}+\left(c_{2}-\frac{g_{A}^{2}}{8 M_{N}}\right) \frac{1}{f_{\pi}^{2}}\left(\partial_{0} \boldsymbol{\pi} \cdot \partial_{0} \boldsymbol{\pi}\right)\right. \\
& \left.+\frac{c_{3}}{f_{\pi}^{2}}\left(\partial_{\mu} \boldsymbol{\pi} \cdot \partial^{\mu} \boldsymbol{\pi}\right)-\left(c_{4}+\frac{1}{4 M_{N}}\right) \frac{1}{2 f_{\pi}^{2}} \epsilon^{i j k} \epsilon^{a b c} \sigma^{i} \tau^{a}\left(\partial^{j} \pi^{b}\right)\left(\partial^{k} \pi^{c}\right)\right] N \\
& -\frac{D}{4 f_{\pi}}(\bar{N} N) \bar{N}[\boldsymbol{\tau} \cdot(\vec{\sigma} \cdot \vec{\nabla}) \boldsymbol{\pi}] N \\
& -\frac{1}{2} E(\bar{N} N)(\bar{N} \boldsymbol{\tau} N) \cdot(\bar{N} \boldsymbol{\tau} N)+\ldots, \\
\widehat{\mathcal{L}}^{\Delta=2}= & \mathcal{L}_{\pi \pi}^{(4)}+\widehat{\mathcal{L}}_{\pi N}^{(3)}+\widehat{\mathcal{L}}_{N N}^{(2)}+\ldots, \\
\widehat{\mathcal{L}}^{\Delta=3}= & \widehat{\mathcal{L}}_{\pi N}^{(4)}+\ldots, \\
\widehat{\mathcal{L}}^{\Delta=4}= & \widehat{\mathcal{L}}_{\pi N}^{(5)}+\widehat{\mathcal{L}}_{N N}^{(4)}+\ldots \\
\widehat{\mathcal{L}}^{\Delta=6}= & \widehat{\mathcal{L}}_{N N}^{(6)}+\ldots,
\end{aligned}
$$

where we included terms relevant for a calculation of the two-nucleon force up to sixth order. The Lagrangians $\widehat{\mathcal{L}}_{\pi N}^{(3)}$ and $\widehat{\mathcal{L}}_{\pi N}^{(4)}$ can be found in Ref. [22] and $N N$ contact 
Lagrangians are given below. The pion fields are denoted by $\boldsymbol{\pi}$ and the heavy baryon nucleon field by $N\left(\bar{N}=N^{\dagger}\right)$. Furthermore, $g_{A}, f_{\pi}, m_{\pi}$, and $M_{N}$ are the axial-vector coupling constant, pion decay constant, pion mass, and nucleon mass, respectively. Numerical values for these quantities will be given later. The $c_{i}$ are low-energy constants (LECs) from the dimension two $\pi N$ Lagrangian and $\alpha$ is a parameter that appears in the expansion of a $S U(2)$ matrix $U$ in powers of the pion fields, see Ref. [11] for more details. Results are independent of $\alpha$.

The lowest order (or leading order) $N N$ Lagrangian has no derivatives and reads [9]

$$
\widehat{\mathcal{L}}_{N N}^{(0)}=-\frac{1}{2} C_{S} \bar{N} N \bar{N} N-\frac{1}{2} C_{T}(\bar{N} \vec{\sigma} N) \cdot(\bar{N} \vec{\sigma} N)
$$

where $C_{S}$ and $C_{T}$ are free paramters to be determined by fitting to the $N N$ data.

The second order $N N$ Lagrangian can be stated as follows [23]

$$
\begin{aligned}
\widehat{\mathcal{L}}_{N N}^{(2)}= & -C_{1}^{\prime}\left[(\bar{N} \vec{\nabla} N)^{2}+(\overline{\vec{\nabla} N} N)^{2}\right]-C_{2}^{\prime}(\bar{N} \vec{\nabla} N) \cdot(\overline{\vec{\nabla} N} N) \\
& -C_{3}^{\prime} \bar{N} N\left[\bar{N} \vec{\nabla}^{2} N+\overline{\vec{\nabla}^{2} N} N\right] \\
& -i C_{4}^{\prime}[\bar{N} \vec{\nabla} N \cdot(\overline{\vec{\nabla} N} \times \vec{\sigma} N)+\overline{(\vec{\nabla} N)} N \cdot(\bar{N} \vec{\sigma} \times \vec{\nabla} N)] \\
& -i C_{5}^{\prime} \bar{N} N(\overline{\vec{\nabla} N} \cdot \vec{\sigma} \times \vec{\nabla} N)-i C_{6}^{\prime}(\bar{N} \vec{\sigma} N) \cdot(\overline{\vec{\nabla} N} \times \vec{\nabla} N) \\
& -\left(C_{7}^{\prime} \delta_{i k} \delta_{j l}+C_{8}^{\prime} \delta_{i l} \delta_{k j}+C_{9}^{\prime} \delta_{i j} \delta_{k l}\right) \\
& \times\left[\bar{N} \sigma_{k} \partial_{i} N \bar{N} \sigma_{l} \partial_{j} N+\overline{\partial_{i} N} \sigma_{k} N \overline{\partial_{j} N} \sigma_{l} N\right] \\
& -\left(C_{10}^{\prime} \delta_{i k} \delta_{j l}+C_{11}^{\prime} \delta_{i l} \delta_{k j}+C_{12}^{\prime} \delta_{i j} \delta_{k l}\right) \bar{N} \sigma_{k} \partial_{i} N \overline{\partial_{j} N} \sigma_{l} N \\
& -\left(\frac{1}{2} C_{13}^{\prime}\left(\delta_{i k} \delta_{j l}+\delta_{i l} \delta_{k j}\right)+C_{14}^{\prime} \delta_{i j} \delta_{k l}\right) \\
& \times\left[\overline{\partial_{i} N} \sigma_{k} \partial_{j} N+\overline{\partial_{j} N} \sigma_{k} \partial_{i} N\right] \bar{N} \sigma_{l} N .
\end{aligned}
$$

Similar to $C_{S}$ and $C_{T}$ of Eq. (23), the $C_{i}^{\prime}$ of Eq. (24) are free parameters which are determined in a fit to the $N N$ data. Clearly, the contact Lagrangians grow considerably in size and complexity as the order increases. Therefore we do not provide here explicit expressions for $\widehat{\mathcal{L}}_{N N}^{(4)}$ and $\widehat{\mathcal{L}}_{N N}^{(6)}$. The $N N$ contact potentials derived from some of the $N N$ Lagrangians are given in Sec. 6.1.

\section{Nuclear forces from EFT: Overview}

We proceed here with discussing the various steps towards a derivation of nuclear forces from EFT. In this section, we will discuss the expansion we are using in more details as well as the various Feynman diagrams as they emerge at each order.

\subsection{Chiral perturbation theory and power counting}

An infinite number of Feynman diagrams can be evaluated from the effective Langrangians and so one needs to be able to organize these diagrams in order of their importance. Chiral perturbation theory provides such organizational scheme. 
In ChPT, graphs are analyzed in terms of powers of small external momenta over the large scale: $\left(Q / \Lambda_{\chi}\right)^{\nu}$, where $Q$ is generic for a momentum (nucleon three-momentum or pion four-momentum) or a pion mass and $\Lambda_{\chi} \sim 1 \mathrm{GeV}$ is the chiral symmetry breaking scale (hadronic scale, hard scale). Determining the power $\nu$ has become known as power counting.

For the moment, we will consider only so-called irreducible graphs. By definition, an irreducible graph is a diagram that cannot be separated into two by cutting only nucleon lines. Following the Feynman rules of covariant perturbation theory, a nucleon propagator carries the dimension $Q^{-1}$, a pion propagator $Q^{-2}$, each derivative in any interaction is $Q$, and each four-momentum integration $Q^{4}$. This is also known as naive dimensional analysis. Applying then some topological identities, one obtains for the power of an irreducible diagram involving $A$ nucleons [11]

$$
\nu=-2+2 A-2 C+2 L+\sum_{i} \Delta_{i}
$$

with

$$
\Delta_{i} \equiv d_{i}+\frac{n_{i}}{2}-2
$$

In the two equations above: for each vertex $i, C$ represents the number of individually connected parts of the diagram while $L$ is the number of loops; $d_{i}$ indicates how many derivatives or pion masses are present and $n_{i}$ the number of nucleon fields. The summation extends over all vertices present in that particular diagram. Notice also that chiral symmetry implies $\Delta_{i} \geq 0$. Interactions among pions have at least two derivatives $\left(d_{i} \geq 2, n_{i}=0\right)$, while interactions between pions and a nucleon have one or more derivatives $\left(d_{i} \geq 1, n_{i}=2\right)$. Finally, pure contact interactions among nucleons $\left(n_{i}=4\right)$ have $d_{i} \geq 0$. In this way, a low-momentum expansion based on chiral symmetry can be constructed.

Naturally, the powers must be bounded from below for the expansion to converge. This is in fact the case, with $\nu \geq 0$.

Furthermore, the power formula Eq. (25) allows to predict the leading orders of connected multi-nucleon forces. Consider a $m$-nucleon irreducibly connected diagram ( $m$-nucleon force) in an $A$-nucleon system $(m \leq A)$. The number of separately connected pieces is $C=A-m+1$. Inserting this into Eq. (25) together with $L=0$ and $\sum_{i} \Delta_{i}=0$ yields $\nu=2 m-4$. Thus, two-nucleon forces $(m=2)$ appear at $\nu=0$, three-nucleon forces $(m=3)$ at $\nu=2$ (but they happen to cancel at that order), and four-nucleon forces at $\nu=4$ (they don't cancel). More about this in the next sub-section.

For later purposes, we note that for an irreducible $N N$ diagram $(A=2, C=1)$, the power formula collapses to the very simple expression

$$
\nu=2 L+\sum_{i} \Delta_{i}
$$

To summarize, at each order $\nu$ we only have a well defined number of diagrams, which renders the theory feasible from a practical standpoint. The magnitude of what has been left out at order $\nu$ can be estimated (in a very simple way) from $\left(Q / \Lambda_{\chi}\right)^{\nu+1}$. 


\section{N Force}

3N Force

4N Force

5N Force

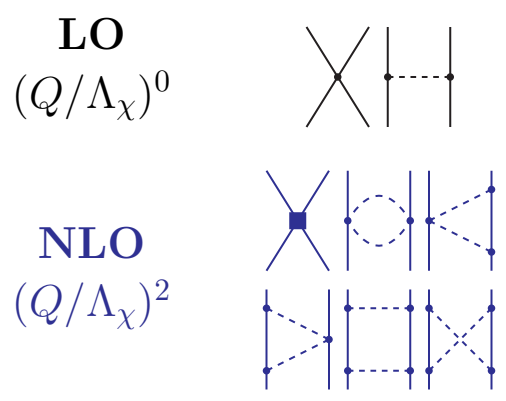

NNLO

$\left(Q / \Lambda_{\chi}\right)^{3}$
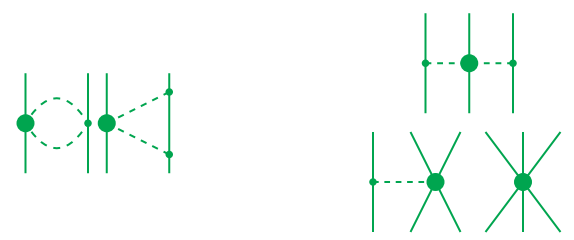

$\mathrm{N}^{3} \mathbf{L O}$
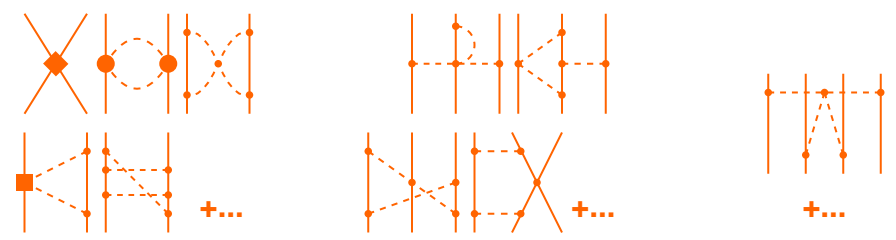

$\left(Q / \Lambda_{\chi}\right)^{4}$

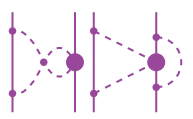

$\mathbf{N}^{4} \mathbf{L O}$
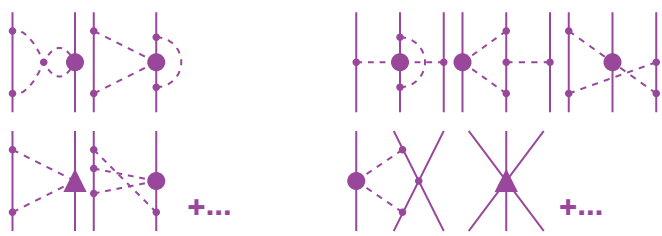

$\left(Q / \Lambda_{\chi}\right)^{5}$
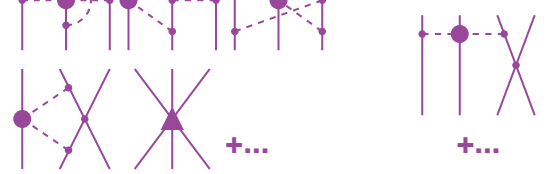

$\mathbf{N}^{5} \mathbf{L O}$
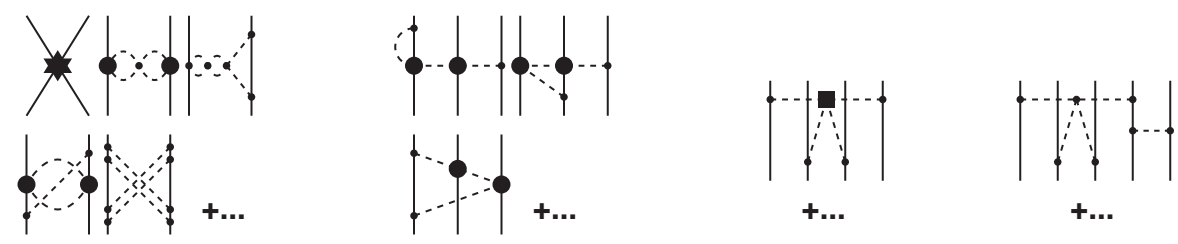

Figure 1. Hierarchy of nuclear forces in ChPT. Solid lines represent nucleons and dashed lines pions. Small dots, large solid dots, solid squares, triangles, diamonds, and stars denote vertices of index $\Delta=0,1,2,3,4$, and 6 , respectively. Further explanations are given in the text.

The ability to calculate observables (in principle) to any degree of accuracy gives the theory its predictive power.

\subsection{The ranking of nuclear forces}

As shown in Fig. 1, nuclear forces appear in ranked orders in accordance with the power counting scheme.

The lowest power is $\nu=0$, also known as the leading order (LO). At LO we have only two contact contributions with no momentum dependence $\left(\sim Q^{0}\right)$. They are 
signified by the four-nucleon-leg diagram with a small-dot vertex shown in the first row of Fig. 1. Besides this, we have the static one-pion exchange (1PE), also shown in the first row of Fig. 1 .

In spite of its simplicity, this rough description contains some of the main attributes of the $N N$ force. First, through the 1PE it generates the tensor component of the force known to be crucial for the two-nucleon bound state. Second, it predict correctly $N N$ phase parameters for high partial waves. At LO, the two terms which result from a partial-wave expansion of the contact term impact states of zero orbital angular momentum and produce attraction at short- and intermediate-range.

Notice that that there are no terms with power $\nu=1$, as they would violate parity conservation and time-reversal invariance.

The next order is then $\nu=2$, next-to-leading order, or NLO.

Note that the two-pion exchange (2PE) makes its first appearance at this order, and thus it is referred to as the "leading 2PE". As is well known from decades of nuclear physics, this contribution is essential for a realistic account of the intermediaterange attraction. However, the leading $2 \mathrm{PE}$ has insufficient strength, for the following reason: the loops present in the diagrams which involve pions carry the power $\nu=2$ [cf. Eq. (27)], and so only $\pi N N$ and $\pi \pi N N$ vertices with $\Delta_{i}=0$ are allowed at this order. These vertices are known to be weak. Moreover, seven new contacts appear at this order which impact $L=0$ and $L=1$ states. (As always, two-nucleon contact terms are indicated by four-nucleon-leg diagrams and a vertex of appropriate shape, in this case a solid square.) At this power, the appropriate operators include spin-orbit, central, spin-spin, and tensor terms, namely all the spin and isospin operator structures needed for a realistic description of the $2 \mathrm{NF}$, although the medium-range attraction still lacks sufficient strength.

At the next order, $\nu=3$ or next-to-next-to-leading order (NNLO), the 2PE contains the so-called $\pi \pi N N$ seagull vertices with two derivatives. These vertices (proportional to the $c_{i}$ LECs and denoted by a large solid dot in Fig. 1), bring in correlated $2 \mathrm{PE}$ and intermediate $\Delta(1232)$-isobar contributions. Consistent with what meson theory of the nuclear force [6, 7] has shown since a long time concerning the importance of these effects, at this order the $2 \mathrm{PE}$ finally provides medium-range attraction of realistic strength, bringing the description of the $N N$ force to an almost quantitative level. No new contacts become available at NNLO.

The discussion above reveals how two- and many-nucleon forces are generated and increase in number as we move to higher orders. Three-nucleon forces appear at NLO, but their net contribution vanishes at this order [10]. The first non-zero 3NF contribution is found at NNLO [24, 25]. It is therefore easy to understand why $3 \mathrm{NF}$ are very weak as compared to the $2 \mathrm{NF}$ which contributes already at $\left(Q / \Lambda_{\chi}\right)^{0}$.

For $\nu=4$, or next-to-next-to-next-to-leading order $\left(\mathrm{N}^{3} \mathrm{LO}\right)$, we display some representative diagrams in Fig. 1. There is a large attractive one-loop 2PE contribution (the bubble diagram with two large solid dots $\sim c_{i}^{2}$ ), which slightly over-estimates the $2 \mathrm{NF}$ attraction at medium range. Two-pion-exchange graphs with two loops are seen 
at this order, together with three-pion exchange $(3 \mathrm{PE})$, which was determined to be very weak at $\mathrm{N}^{3} \mathrm{LO}$ [26, 27]. The most important feature at this order is the presence of 15 additional contacts $\sim Q^{4}$, signified by the four-nucleon-leg diagram in the figure with the diamond-shaped vertex. These contacts impact states with orbital angular momentum up to $L=2$, and are the reason for the quantitative description of the two-nucleon force (up to approximately $300 \mathrm{MeV}$ in terms of laboratory energy) at this order [11, 28]. More 3NF diagrams show up at $\mathrm{N}^{3} \mathrm{LO}$, as well as the first contributions to four-nucleon forces $(4 \mathrm{NF})$. We then see that forces involving more and more nucleons appear for the first time at higher and higher orders, which gives theoretical support to the fact that $2 \mathrm{NF} \gg 3 \mathrm{NF} \gg 4 \mathrm{NF} \ldots$

Further $2 \mathrm{PE}$ and $3 \mathrm{PE}$ occur at $\mathrm{N}^{4} \mathrm{LO}$ (fifth order). The contribution to the $2 \mathrm{NF}$ at this order has been first calculated by Entem et al. [29]. It turns out to be moderately repulsive, thus compensating for the attractive surplus generated at $\mathrm{N}^{3} \mathrm{LO}$ by the bubble diagram with two solid dots. The long- and intermediate-range $3 \mathrm{NF}$ contributions at this order have been evaluated [22, 30], but not yet applied in nuclear structure calculations. They are expected to be sizeable. Moreover, a new set of 3NF contact terms appears [31]. The $\mathrm{N}^{4} \mathrm{LO} 4 \mathrm{NF}$ has not been derived yet. Due to the subleading $\pi \pi N N$ seagull vertex (large solid dot $\sim c_{i}$ ), this $4 \mathrm{NF}$ could be sizeable.

Finally turning to $\mathrm{N}^{5} \mathrm{LO}$ (sixth order): The dominant $2 \mathrm{PE}$ and $3 \mathrm{PE}$ contributions to the $2 \mathrm{NF}$ have been derived by Entem et al. in Ref. [32], which represents the most sophisticated investigation ever conducted in chiral EFT for the $N N$ system. The effects are small indicating the desired trend towards convergence of the chiral expansion for the $2 \mathrm{NF}$. Moreover, a new set of $26 N N$ contact terms $\sim Q^{6}$ occurs that contributes up to $F$-waves (represented by the $N N$ diagram with a star in Fig. 1 ) bringing the total number of $N N$ contacts to 50 [33]. The three-, four-, and five-nucleon forces of this order have not yet been derived.

This section has provided an overview. In the following sections, we will present more details.

\section{Pion-exchange contributions to the $N N$ interaction}

The various pion-exchange contributions to the $N N$ potential may be analyzed according to the number of pions being exchanged between the two nucleons:

$$
V=V_{1 \pi}+V_{2 \pi}+V_{3 \pi}+V_{4 \pi}+\ldots
$$

where the meaning of the subscripts is obvious and the ellipsis represents $5 \pi$ and higher pion exchanges. For each of the above terms, we assume a low-momentum expansion:

$$
\begin{aligned}
& V_{1 \pi}=V_{1 \pi}^{(0)}+V_{1 \pi}^{(2)}+V_{1 \pi}^{(3)}+V_{1 \pi}^{(4)}+V_{1 \pi}^{(5)}+V_{1 \pi}^{(6)}+\ldots \\
& V_{2 \pi}=V_{2 \pi}^{(2)}+V_{2 \pi}^{(3)}+V_{2 \pi}^{(4)}+V_{2 \pi}^{(5)}+V_{2 \pi}^{(6)}+\ldots \\
& V_{3 \pi}=V_{3 \pi}^{(4)}+V_{3 \pi}^{(5)}+V_{3 \pi}^{(6)}+\ldots \\
& V_{4 \pi}=V_{4 \pi}^{(6)}+\ldots
\end{aligned}
$$


where the superscript denotes the order $\nu$ of the expansion and the ellipses stand for contributions of seventh and higher orders. Due to parity and time-reversal, there are no first order contributions. Moreover, since $n$ pions create $L=n-1$ loops, the leading order for $n$-pion exchange ocurrs at $\nu=2 n-2$ [cf. Eq. (27)].

Order by order, the pion-exchange part of the $N N$ potential builds up as follows:

$$
\begin{aligned}
& V_{\mathrm{LO}} \equiv V^{(0)}=V_{1 \pi}^{(0)} \\
& V_{\mathrm{NLO}} \equiv V^{(2)}=V_{\mathrm{LO}}+V_{1 \pi}^{(2)}+V_{2 \pi}^{(2)} \\
& V_{\mathrm{NNLO}} \equiv V^{(3)}=V_{\mathrm{NLO}}+V_{1 \pi}^{(3)}+V_{2 \pi}^{(3)} \\
& V_{\mathrm{N} 3 \mathrm{LO}} \equiv V^{(4)}=V_{\mathrm{NNLO}}+V_{1 \pi}^{(4)}+V_{2 \pi}^{(4)}+V_{3 \pi}^{(4)} \\
& V_{\mathrm{N} 4 \mathrm{LO}} \equiv V^{(5)}=V_{\mathrm{N} 3 \mathrm{LO}}+V_{1 \pi}^{(5)}+V_{2 \pi}^{(5)}+V_{3 \pi}^{(5)} \\
& V_{\mathrm{N} 5 \mathrm{LO}} \equiv V^{(6)}=V_{\mathrm{N} 4 \mathrm{LO}}+V_{1 \pi}^{(6)}+V_{2 \pi}^{(6)}+V_{3 \pi}^{(6)}+V_{4 \pi}^{(6)}
\end{aligned}
$$

where LO stands for leading order, NLO for next-to-leading order, etc..

The explicit expressions for the potentials will be stated in terms of contributions to the momentum-space $N N$ amplitudes in the center-of-mass system (CMS), which arise from the following general decomposition:

$$
\begin{aligned}
V\left(\vec{p}^{\prime}, \vec{p}\right) & =V_{C}+\boldsymbol{\tau}_{1} \cdot \boldsymbol{\tau}_{2} W_{C} \\
& +\left[V_{S}+\boldsymbol{\tau}_{1} \cdot \boldsymbol{\tau}_{2} W_{S}\right] \vec{\sigma}_{1} \cdot \vec{\sigma}_{2} \\
& +\left[V_{L S}+\boldsymbol{\tau}_{1} \cdot \boldsymbol{\tau}_{2} W_{L S}\right](-i \vec{S} \cdot(\vec{q} \times \vec{k})) \\
& +\left[V_{T}+\boldsymbol{\tau}_{1} \cdot \boldsymbol{\tau}_{2} W_{T}\right] \vec{\sigma}_{1} \cdot \vec{q} \vec{\sigma}_{2} \cdot \vec{q} \\
& +\left[V_{\sigma L}+\boldsymbol{\tau}_{1} \cdot \boldsymbol{\tau}_{2} W_{\sigma L}\right] \vec{\sigma}_{1} \cdot(\vec{q} \times \vec{k}) \vec{\sigma}_{2} \cdot(\vec{q} \times \vec{k}),
\end{aligned}
$$

where $\vec{p}^{\prime}$ and $\vec{p}$ denote the final and initial nucleon momenta in the CMS, respectively. Moreover, $\vec{q}=\vec{p}^{\prime}-\vec{p}$ is the momentum transfer, $\vec{k}=\left(\vec{p}^{\prime}+\vec{p}\right) / 2$ the average momentum, and $\vec{S}=\left(\vec{\sigma}_{1}+\vec{\sigma}_{2}\right) / 2$ the total spin, with $\vec{\sigma}_{1,2}$ and $\boldsymbol{\tau}_{1,2}$ the spin and isospin operators of nucleon 1 and 2, respectively. For on-shell scattering, $V_{\alpha}$ and $W_{\alpha}(\alpha=C, S, L S, T, \sigma L)$ can be expressed as functions of $q=|\vec{q}|$ and $p=\left|\vec{p}^{\prime}\right|=|\vec{p}|$, only.

We will now discuss the contributions order by order.

\subsection{Leading order (LO)}

At leading order, there is only the $1 \pi$-exchange contribution, cf. Fig. 1. The chargeindependent $1 \pi$-exchange is given by

$$
V_{1 \pi}^{(\mathrm{CI})}\left(\vec{p}^{\prime}, \vec{p}\right)=-\frac{g_{A}^{2}}{4 f_{\pi}^{2}} \boldsymbol{\tau}_{1} \cdot \boldsymbol{\tau}_{2} \frac{\vec{\sigma}_{1} \cdot \vec{q} \vec{\sigma}_{2} \cdot \vec{q}}{q^{2}+m_{\pi}^{2}}
$$

Higher order corrections to the $1 \pi$-exchange are taken care of by mass and coupling constant renormalizations which, in turn, are accounted for by working with the physical values. Note also that, on shell, there are no relativistic corrections. Thus, we apply $1 \pi$-exchange in the form Eq. 40 through all orders.

We use $g_{A}=1.290$ (instead of $g_{A}=1.276$ [34]) to account for the so-called Goldberger-Treiman discrepancy. Via the Goldberger-Treiman relation, $g_{\pi N N}=$ 
$g_{A} M_{N} / f_{\pi}$, our value for $g_{A}$ together with $f_{\pi}=92.4 \mathrm{MeV}$ and $M_{N}=938.918 \mathrm{MeV}$ implies $g_{\pi N N}^{2} / 4 \pi=13.67$ which is consistent with the empirical values obtained from $\pi N$ and $N N$ data analysis [35, 36].

For results presented below, we will be specifically calculating neutron-proton $(n p)$ scattering and take the charge-dependence of the $1 \pi$-exchange into account. Thus, the $1 \pi$-exchange potential that we actually apply reads

$$
V_{1 \pi}^{(n p)}\left(\vec{p}^{\prime}, \vec{p}\right)=-V_{1 \pi}\left(m_{\pi^{0}}\right)+(-1)^{I+1} 2 V_{1 \pi}\left(m_{\pi^{ \pm}}\right),
$$

where $I=0,1$ denotes the total isospin of the two-nucleon system and

$$
V_{1 \pi}\left(m_{\pi}\right) \equiv-\frac{g_{A}^{2}}{4 f_{\pi}^{2}} \frac{\vec{\sigma}_{1} \cdot \vec{q} \vec{\sigma}_{2} \cdot \vec{q}}{q^{2}+m_{\pi}^{2}}
$$

We use $m_{\pi^{0}}=134.9766 \mathrm{MeV}$ and $m_{\pi^{ \pm}}=139.5702 \mathrm{MeV}$. Formally speaking, the chargedependence of the 1PE exchange is of order NLO [11], but we include it already at leading order to make the comparison with the $n p$ phase shifts more meaningful.

\subsection{Next-to-leading order (NLO)}

The $N N$ diagrams that occur at NLO (cf. Fig. 1) contribute in the following way [37]:

$$
\begin{aligned}
& W_{C}=\frac{L(\tilde{\Lambda} ; q)}{384 \pi^{2} f_{\pi}^{4}}\left[4 m_{\pi}^{2}\left(1+4 g_{A}^{2}-5 g_{A}^{4}\right)+q^{2}\left(1+10 g_{A}^{2}-23 g_{A}^{4}\right)-\frac{48 g_{A}^{4} m_{\pi}^{4}}{w^{2}}\right], \\
& V_{T}=-\frac{1}{q^{2}} V_{S}=-\frac{3 g_{A}^{4}}{64 \pi^{2} f_{\pi}^{4}} L(\tilde{\Lambda} ; q),
\end{aligned}
$$

where the (regularized) logarithmic loop function is given by:

$$
L(\tilde{\Lambda} ; q)=\frac{w}{2 q} \ln \frac{\tilde{\Lambda}^{2}\left(2 m_{\pi}^{2}+q^{2}\right)-2 m_{\pi}^{2} q^{2}+\tilde{\Lambda} \sqrt{\tilde{\Lambda}^{2}-4 m_{\pi}^{2}} q w}{2 m_{\pi}^{2}\left(\tilde{\Lambda}^{2}+q^{2}\right)}
$$

with $w=\sqrt{4 m_{\pi}^{2}+q^{2}}$. $\tilde{\Lambda}$ denotes the cutoff of the spectral-function renormalization (SFR) 38. Note that

$$
\lim _{\tilde{\Lambda} \rightarrow \infty} L(\tilde{\Lambda} ; q)=\frac{w}{q} \ln \frac{w+q}{2 m_{\pi}},
$$

is the logarithmic loop function of dimensional regularization.

\subsection{Next-to-next-to-leading order (NNLO)}

The NNLO contribution (cf. the $2 \mathrm{NF}$ diagrams of the NNLO row in Fig. 1) is given by [37:

$$
\begin{aligned}
& V_{C}=\frac{3 g_{A}^{2}}{16 \pi f_{\pi}^{4}}\left[2 m_{\pi}^{2}\left(c_{3}-2 c_{1}\right)+c_{3} q^{2}\right]\left(2 m_{\pi}^{2}+q^{2}\right) A(\tilde{\Lambda} ; q), \\
& W_{T}=-\frac{1}{q^{2}} W_{S}=-\frac{g_{A}^{2}}{32 \pi f_{\pi}^{4}} c_{4} w^{2} A(\tilde{\Lambda} ; q) .
\end{aligned}
$$


The loop function that appears in the above expressions, regularized by spectral-function cut-off $\tilde{\Lambda}$, is

$$
A(\tilde{\Lambda} ; q)=\frac{1}{2 q} \arctan \frac{q\left(\tilde{\Lambda}-2 m_{\pi}\right)}{q^{2}+2 \tilde{\Lambda} m_{\pi}},
$$

and

$$
\lim _{\tilde{\Lambda} \rightarrow \infty} A(\tilde{\Lambda} ; q)=\frac{1}{2 q} \arctan \frac{q}{2 m_{\pi}}
$$

yields the loop function used in dimensional regularization.

\subsection{Next-to-next-to-next-to-leading order ( $\left.N^{3} L O\right)$}

The number of diagrams involved is now dramatically increasing. Therefore, we will provide additional figures showing the full complexity of the diagrams representing the nuclear forces at higher orders.

The 2PE contributions at $\mathrm{N}^{3} \mathrm{LO}$ are shown in Fig. 2. They consist of three parts, which we will discuss one by one.

4.4.1. Football diagram at $N^{3} L O$ The football diagram at $\mathrm{N}^{3} \mathrm{LO}$, Fig. 2(a), generates [39]:

$$
\begin{aligned}
& V_{C}=\frac{3}{16 \pi^{2} f_{\pi}^{4}}\left[\left(\frac{c_{2}}{6} w^{2}+c_{3}\left(2 m_{\pi}^{2}+q^{2}\right)-4 c_{1} m_{\pi}^{2}\right)^{2}+\frac{c_{2}^{2}}{45} w^{4}\right] L(\tilde{\Lambda} ; q), \\
& W_{T}=-\frac{1}{q^{2}} W_{S}=\frac{c_{4}^{2}}{96 \pi^{2} f_{\pi}^{4}} w^{2} L(\tilde{\Lambda} ; q) .
\end{aligned}
$$

4.4.2. Leading two-loop contributions The leading order $2 \pi$-exchange two-loop diagrams are shown in Fig. 2(b). In terms of spectral functions, the results are [39]:

$$
\begin{aligned}
\operatorname{Im} V_{C}(i \mu)= & \frac{3 g_{A}^{4}\left(2 m_{\pi}^{2}-\mu^{2}\right)}{\pi \mu\left(4 f_{\pi}\right)^{6}}\left[\left(m_{\pi}^{2}-2 \mu^{2}\right)\left(2 m_{\pi}+\frac{2 m_{\pi}^{2}-\mu^{2}}{2 \mu} \ln \frac{\mu+2 m_{\pi}}{\mu-2 m_{\pi}}\right)\right. \\
& \left.+4 g_{A}^{2} m_{\pi}\left(2 m_{\pi}^{2}-\mu^{2}\right)\right] \\
\operatorname{Im} W_{C}(i \mu)= & \frac{2 \kappa}{3 \mu\left(8 \pi f_{\pi}^{2}\right)^{3}} \int_{0}^{1} d x\left[g_{A}^{2}\left(\mu^{2}-2 m_{\pi}^{2}\right)+2\left(1-g_{A}^{2}\right) \kappa^{2} x^{2}\right] \\
& \times\left\{96 \pi^{2} f_{\pi}^{2}\left[\left(2 m_{\pi}^{2}-\mu^{2}\right)\left(\bar{d}_{1}+\bar{d}_{2}\right)-2 \kappa^{2} x^{2} \bar{d}_{3}+4 m_{\pi}^{2} \bar{d}_{5}\right]\right. \\
& +\left[4 m_{\pi}^{2}\left(1+2 g_{A}^{2}\right)-\mu^{2}\left(1+5 g_{A}^{2}\right)\right] \frac{\kappa}{\mu} \ln \frac{\mu+2 \kappa}{2 m_{\pi}} \\
& +\frac{\mu^{2}}{12}\left(5+13 g_{A}^{2}\right)-2 m_{\pi}^{2}\left(1+2 g_{A}^{2}\right)-3 \kappa^{2} x^{2} \\
& +6 \kappa x \sqrt{m_{\pi}^{2}+\kappa^{2} x^{2}} \ln \frac{\kappa x+\sqrt{m_{\pi}^{2}+\kappa^{2} x^{2}}}{m_{\pi}} \\
& +g_{A}^{4}\left(\mu^{2}-2 \kappa^{2} x^{2}-2 m_{\pi}^{2}\right)
\end{aligned}
$$




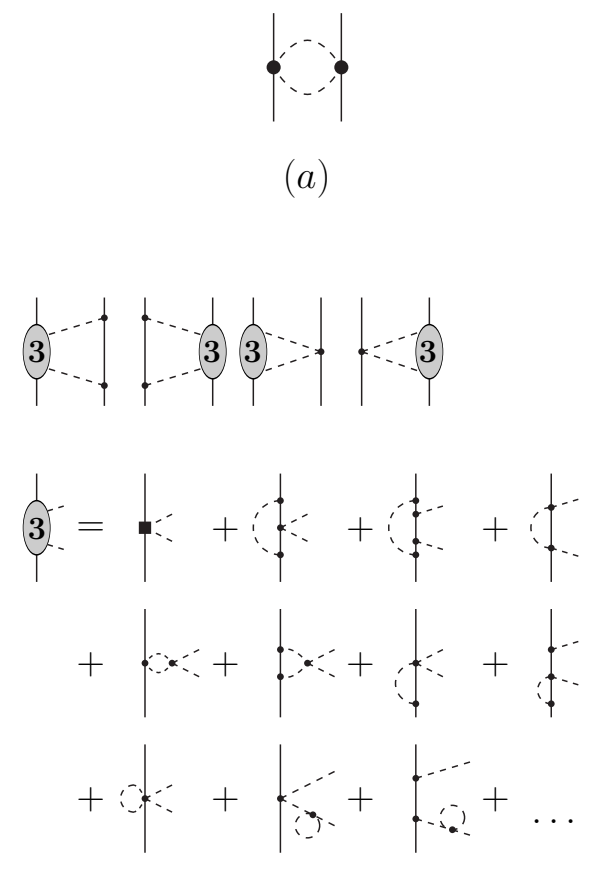

(b)
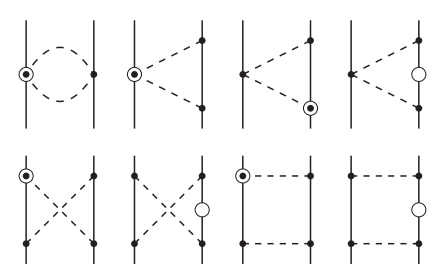

(c)

Figure 2. $\mathrm{N}^{3} \mathrm{LO}$ two-pion exchange contributions with (a) the $\mathrm{N}^{3} \mathrm{LO}$ football diagram, (b) the leading 2PE two-loop contributions, and (c) the relativistic corrections of NLO diagrams. Notation as in Fig. 1. Shaded ovals represent complete $\pi N$-scattering amplitudes with their order specified by the number in the oval. Open circles denote relativistic $1 / M_{N}$ corrections.

$$
\left.\times\left[\frac{5}{6}+\frac{m_{\pi}^{2}}{\kappa^{2} x^{2}}-\left(1+\frac{m_{\pi}^{2}}{\kappa^{2} x^{2}}\right)^{3 / 2} \ln \frac{\kappa x+\sqrt{m_{\pi}^{2}+\kappa^{2} x^{2}}}{m_{\pi}}\right]\right\}
$$

$\operatorname{Im} V_{S}(i \mu)=\mu^{2} \operatorname{Im} V_{T}(i \mu)=\frac{g_{A}^{2} \mu \kappa^{3}}{8 \pi f_{\pi}^{4}}\left(\bar{d}_{15}-\bar{d}_{14}\right)$

$$
\begin{aligned}
& +\frac{2 g_{A}^{6} \mu \kappa^{3}}{\left(8 \pi f_{\pi}^{2}\right)^{3}} \int_{0}^{1} d x\left(1-x^{2}\right)\left[\frac{1}{6}-\frac{m_{\pi}^{2}}{\kappa^{2} x^{2}}+\left(1+\frac{m_{\pi}^{2}}{\kappa^{2} x^{2}}\right)^{3 / 2}\right. \\
& \left.\times \ln \frac{\kappa x+\sqrt{m_{\pi}^{2}+\kappa^{2} x^{2}}}{m_{\pi}}\right],
\end{aligned}
$$

$\operatorname{Im} W_{S}(i \mu)=\mu^{2} \operatorname{Im} W_{T}(i \mu)=\frac{g_{A}^{4}\left(4 m_{\pi}^{2}-\mu^{2}\right)}{\pi\left(4 f_{\pi}\right)^{6}}\left[\left(m_{\pi}^{2}-\frac{\mu^{2}}{4}\right) \ln \frac{\mu+2 m_{\pi}}{\mu-2 m_{\pi}}\right.$ 


$$
\left.+\left(1+2 g_{A}^{2}\right) \mu m_{\pi}\right]
$$

where $\kappa=\sqrt{\mu^{2} / 4-m_{\pi}^{2}}$.

The momentum space amplitudes $V_{\alpha}(q)$ and $W_{\alpha}(q)$ are obtained from the above expressions by means of subtracted dispersion integrals:

$$
\begin{aligned}
& V_{C, S}(q)=-\frac{2 q^{m+3}}{\pi} \int_{n m_{\pi}}^{\tilde{\Lambda}} d \mu \frac{\operatorname{Im} V_{C, S}(i \mu)}{\mu^{m+2}\left(\mu^{2}+q^{2}\right)}, \\
& V_{T}(q)=\frac{2 q^{m+1}}{\pi} \int_{n m_{\pi}}^{\tilde{\Lambda}} d \mu \frac{\operatorname{Im} V_{T}(i \mu)}{\mu^{m}\left(\mu^{2}+q^{2}\right)},
\end{aligned}
$$

and similarly for $W_{C, S, T}$. We use $m=3$ for the dispersion integrals that contribute at $\mathrm{N}^{3} \mathrm{LO}$ and $\mathrm{N}^{4} \mathrm{LO}$, and $m=5$ at $\mathrm{N}^{5} \mathrm{LO}$. Moreover, $n=2$ is applied for two-pion exchange and $n=3$ for three-pion exchange. For $\tilde{\Lambda} \rightarrow \infty$ the above dispersion integrals yield the results of dimensional regularization, while for finite $\tilde{\Lambda} \geq n m_{\pi}$ we have what has become known as spectral-function regularization (SFR) [38]. The purpose of the finite scale $\tilde{\Lambda}$ is to constrain the imaginary parts to the low-momentum region where chiral effective field theory is applicable.

4.4.3. Leading relativistic corrections Counting $Q / M_{N} \sim Q^{2} / \Lambda_{\chi}^{2}$, the relativistic corrections of the NLO diagrams, which are shown in Fig. 2(c), are of order $\mathrm{N}^{3} \mathrm{LO}$ and are given by [11]:

$$
\begin{aligned}
V_{C}= & \frac{3 g_{A}^{4}}{128 \pi f_{\pi}^{4} M_{N}}\left[\frac{m_{\pi}^{5}}{2 w^{2}}+\left(2 m_{\pi}^{2}+q^{2}\right)\left(q^{2}-m_{\pi}^{2}\right) A(\tilde{\Lambda} ; q)\right], \\
W_{C}= & \frac{g_{A}^{2}}{64 \pi f_{\pi}^{4} M_{N}}\left\{\frac{3 g_{A}^{2} m_{\pi}^{5}}{2 \omega^{2}}+\left[g_{A}^{2}\left(3 m_{\pi}^{2}+2 q^{2}\right)-2 m_{\pi}^{2}-q^{2}\right]\right. \\
& \left.\times\left(2 m_{\pi}^{2}+q^{2}\right) A(\tilde{\Lambda} ; q)\right\}, \\
V_{T}= & -\frac{1}{q^{2}} V_{S}=\frac{3 g_{A}^{4}}{256 \pi f_{\pi}^{4} M_{N}}\left(5 m_{\pi}^{2}+2 q^{2}\right) A(\tilde{\Lambda} ; q), \\
W_{T}= & -\frac{1}{q^{2}} W_{S}=\frac{g_{A}^{2}}{128 \pi f_{\pi}^{4} M_{N}}\left[g_{A}^{2}\left(3 m_{\pi}^{2}+q^{2}\right)-w^{2}\right] A(\tilde{\Lambda} ; q), \\
V_{L S}= & \frac{3 g_{A}^{4}}{32 \pi f_{\pi}^{4} M_{N}}\left(2 m_{\pi}^{2}+q^{2}\right) A(\tilde{\Lambda} ; q), \\
W_{L S}= & \frac{g_{A}^{2}\left(1-g_{A}^{2}\right)}{32 \pi f_{\pi}^{4} M_{N}} w^{2} A(\tilde{\Lambda} ; q) .
\end{aligned}
$$

4.4.4. Leading three-pion exchange contributions The leading $3 \pi$-exchange contributions that occur at $\mathrm{N}^{3} \mathrm{LO}$ are shown in Fig. 3. They have been calculated in Refs. [26, 27] and are found to be negligible. Therefore, we omit them. 

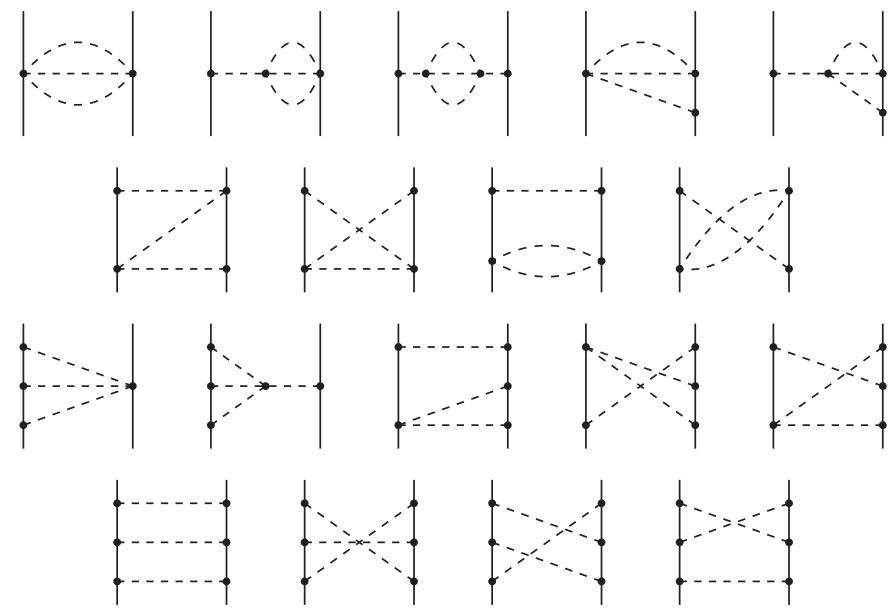

Figure 3. $\mathrm{N}^{3} \mathrm{LO}$ three-pion exchange contributions. Notation as in Fig. 1. (Figure reproduced from Ref. [11.)

\subsection{Next-to-next-to-next-to-next-to-leading order $\left(N^{4} L O\right)$}

At this order, we have two- and three-pion exchange contributions, which we will now discuss one by one.

4.5.1. Two-pion exchange contributions at $N^{4} L O$ The $2 \pi$-exchange contributions that occur at $\mathrm{N}^{4} \mathrm{LO}$ are displayed graphically in Fig. 4. We can distinguish between three groups of diagrams.

First, there are the $\mathrm{N}^{4} \mathrm{LO} 2 \pi$-exchange two-loop contributions of class (a), Fig. 4(a). For this class the spectral functions are obtained by integrating the product of the leading one-loop $\pi N$ amplitude and the chiral $\pi \pi N N$ vertex proportional to $c_{i}$ over the Lorentz-invariant $2 \pi$-phase space.

Second, we have the $\mathrm{N}^{4} \mathrm{LO} 2 \pi$-exchange two-loop contributions of class (b), Fig. 4(b). Here, the product of the one-loop $\pi N$ amplitude proportional to $c_{i}$ (see Ref. [22] for details) and the leading order chiral $\pi N$ amplitude is integrated over the $2 \pi$-phase space.

The analytic expressions for the spectral functions of class (a) and (b) are very involved, which is why we do not reprint them here. The interested reader is referred to Ref. [29].

Finally, there also some relativistic corrections. This group consists of diagrams with one vertex proportional to $c_{i}$ and one $1 / M_{N}$ correction. A few representative graphs are shown in Fig. 4(c). Since in this investigation we count $Q / M_{N} \sim\left(Q / \Lambda_{\chi}\right)^{2}$, these relativistic corrections are formally of order $\mathrm{N}^{4} \mathrm{LO}$. The result for this group of diagrams is [39]:

$$
V_{C}=\frac{g_{A}^{2} L(\tilde{\Lambda} ; q)}{32 \pi^{2} M_{N} f_{\pi}^{4}}\left[\left(6 c_{3}-c_{2}\right) q^{4}+4\left(3 c_{3}-c_{2}-6 c_{1}\right) q^{2} m_{\pi}^{2}\right.
$$




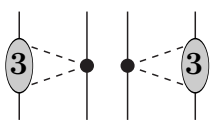

(a)
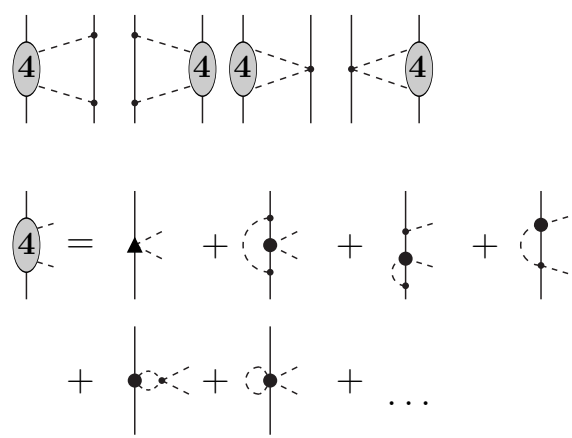

(b)

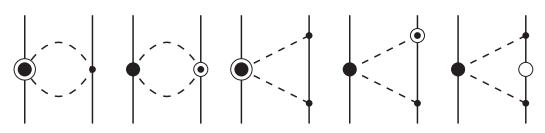

$(c)$

Figure 4. $\mathrm{N}^{4} \mathrm{LO}$ two-pion-exchange contributions. (a) The leading one-loop $\pi N$ amplitude is folded with the chiral $\pi \pi N N$ vertices proportional to $c_{i}$. (b) The one-loop $\pi N$ amplitude proportional to $c_{i}$ is folded with the leading order chiral $\pi N$ amplitude.

(c) Relativistic corrections of NNLO diagrams. Notation as in Figs. 1 and 2 .

$$
\begin{gathered}
\left.+6\left(2 c_{3}-c_{2}\right) m_{\pi}^{4}-24\left(2 c_{1}+c_{3}\right) m_{\pi}^{6} w^{-2}\right] \\
W_{C}=-\frac{c_{4}}{192 \pi^{2} M_{N} f_{\pi}^{4}}\left[g_{A}^{2}\left(8 m_{\pi}^{2}+5 q^{2}\right)+w^{2}\right] q^{2} L(\tilde{\Lambda} ; q) \\
W_{T}=-\frac{1}{q^{2}} W_{S}=\frac{c_{4}}{192 \pi^{2} M_{N} f_{\pi}^{4}}\left[w^{2}-g_{A}^{2}\left(16 m_{\pi}^{2}+7 q^{2}\right)\right] L(\tilde{\Lambda} ; q), \\
V_{L S}=\frac{c_{2} g_{A}^{2}}{8 \pi^{2} M_{N} f_{\pi}^{4}} w^{2} L(\tilde{\Lambda} ; q) \\
W_{L S}=-\frac{c_{4}}{48 \pi^{2} M_{N} f_{\pi}^{4}}\left[g_{A}^{2}\left(8 m_{\pi}^{2}+5 q^{2}\right)+w^{2}\right] L(\tilde{\Lambda} ; q) .
\end{gathered}
$$

4.5.2. Three-pion exchange contributions at $N^{4} L O$ The $3 \pi$-exchange of order $\mathrm{N}^{4} \mathrm{LO}$ is shown in Fig. 5. The spectral functions for these diagrams have been calculated in Ref. [40]. We use here the classification scheme introduced in that reference and note that class XI vanishes. Moreover, we find that the class X and part of class XIV make only negligible contributions. Thus, we include in our calculations only class XII and XIII, and the $V_{S}$ contribution of class XIV. For the very involved expressions, we refer the interested reader to Ref. [29]. 


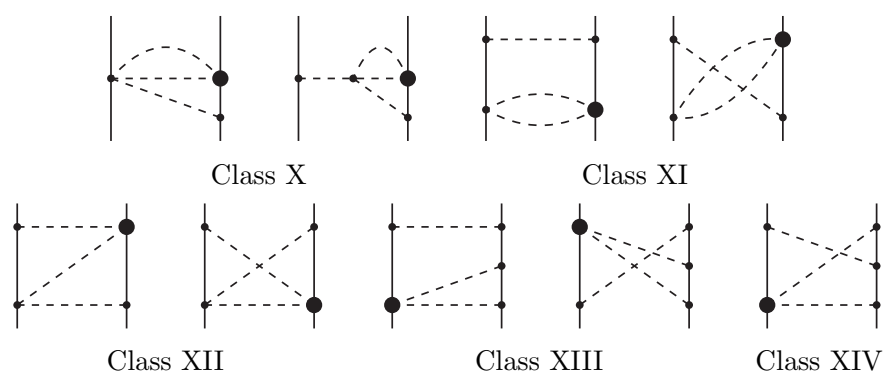

Figure 5. $\mathrm{N}^{4} \mathrm{LO}$ three-pion exchange contributions. Roman numerals refer to subclasses following the scheme introduced in Refs. [40, 29. Notation as in Fig. 1. (Figure reproduced from Ref. [29].)

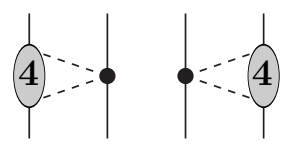

(a)

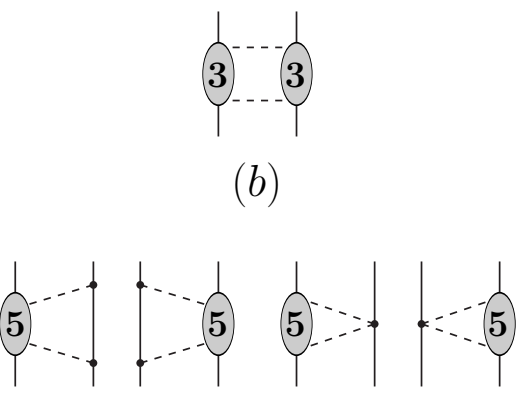

(c)

Figure 6. $\mathrm{N}^{5} \mathrm{LO}$ two-pion-exchange contributions. (a) The subleading one-loop $\pi N$ amplitude is folded with the chiral $\pi \pi N N$-vertices proportional to $c_{i}$. (b) The leading one-loop $\pi N$-amplitude is folded with itself. (c) The leading two-loop $\pi N$-amplitude is folded with the tree-level $\pi N$-amplitude. Notation as in Figs. 1 and 2. (Figure reproduced from Ref. [32].)

\subsection{Next-to-next-to-next-to-next-to-next-to-leading order $\left(N^{5} L O\right)$}

At $\mathrm{N}^{5} \mathrm{LO}$, we are faced with two-, three-, and four-pion exchange contributions.

4.6.1. Two-pion exchange contributions at $N^{5} L O$ The $2 \pi$-exchange contributions that occur at $\mathrm{N}^{5} \mathrm{LO}$ are displayed graphically in Fig. 6. We will now discuss each class separately.

The $\mathrm{N}^{5} \mathrm{LO} 2 \pi$-exchange two-loop contributions, denoted by class (a), are shown in Fig. 6(a). For this class the spectral functions are obtained by integrating the product of the subleading one-loop $\pi N$-amplitude (see Ref. [22] for details) and the chiral $\pi \pi N N$ vertex proportional to $c_{i}$ over the Lorentz-invariant $2 \pi$-phase space [32]. 


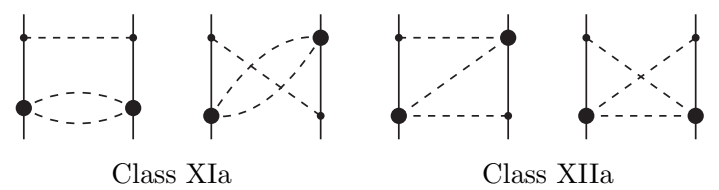

(a)

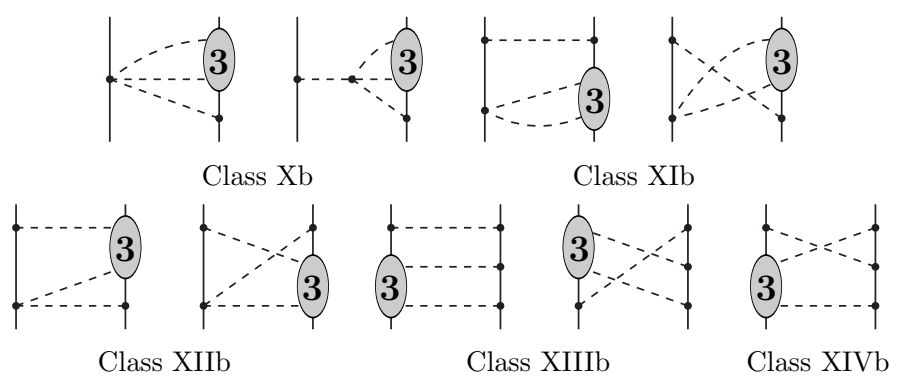

(b)

Figure 7. $\mathrm{N}^{5} \mathrm{LO}$ three-pion exchange contributions. (a) Diagrams proportional to $c_{i}^{2}$. (b) Diagrams involving the one-loop $\pi N$-amplitude. Notation as in Figs. 1, 2, and 5 . (Figure reproduced from Ref. [32.)

A first set of $2 \pi$-exchange contributions at three-loop order, denoted by class (b), is displayed in Fig. 6(b). Here, the leading one-loop $\pi N$-scattering amplitude is multiplied with itself and integrated over the $2 \pi$-phase space [32].

Further $2 \pi$-exchange three-loop contributions at $\mathrm{N}^{5} \mathrm{LO}$, denoted by class (c), are shown in Fig. 6(c). For these, the two-loop $\pi N$-scattering amplitude (which is of order five) would have to be folded with the tree-level $\pi N$-amplitude. To our knowledge, the two-loop elastic $\pi N$-scattering amplitude has never been evaluated in some decent analytical form. Note that the loops involved in the class (c) contributions include only leading order chiral $\pi N$-vertices. According to our experience such contributions are typically small. For these reasons, class (c) is neglected.

Besides the above, there are also some relativistic $1 / M_{N}^{2}$-corrections. This group consists of the $1 / M_{N}^{2}$-corrections to the leading chiral $2 \pi$-exchange diagrams. Since we count $Q / M_{N} \sim\left(Q / \Lambda_{\chi}\right)^{2}$, these relativistic corrections are formally of sixth order $\left(\mathrm{N}^{5} \mathrm{LO}\right)$. The expressions for the corresponding $N N$-amplitudes can be found in Ref. [41].

4.6.2. Three-pion exchange contributions at $N^{5} L O$ The $3 \pi$-exchange contributions of order $\mathrm{N}^{5} \mathrm{LO}$ are shown in Fig. 7. We can distinguish between two classes.

Class (a) consists of the diagrams displayed in Fig. 7(a). They are characterized by the presence of one subleading $\pi \pi N N$-vertex in each nucleon line. Using a notation introduced in Refs. [40, 29], we distinguish between the various sub-classes of diagrams by roman numerals.

Class (b) is shown in Fig. 7(b). Each $3 \pi$-exchange diagram of this class includes the one-loop $\pi N$-amplitude (completed by the low-energy constants $\bar{d}_{j}$ ). Only those parts of 
the $\pi N$-scattering amplitude, which are either independent of the pion CMS-energy or depend on it linearly could be treated with the techniques available. The contributions are, in general, small. Results presented below include only the larger portions within this class. The omitted pieces are about one order of magnitude smaller. To facilitate a better understanding, we have subdivided this class into sub-classes labeled by roman numerals, following Refs. [40, 29].

The very involved analytic expressions for the spectral function can be found in Ref. [32].

4.6.3. Four-pion exchange at $N^{5} L O$ The exchange of four pions between two nucleons occurs for the first time at $\mathrm{N}^{5} \mathrm{LO}$. The pertinent diagrams involve three loops and only leading order vertices, which explains the sixth power in small momenta. Three-pion exchange with just leading order vertices turned out to be negligibly small [26, 27], and so we expect four-pion exchange with leading order vertices to be even smaller. Therefore, we can safely neglect this contribution.

\section{Perturbative $N N$ scattering in peripheral partial waves}

We will now discuss $N N$ scattering involving states of high orbital angular momentum. We recall that peripheral scattering is the best tool to explore the $N N$ force beyond short distances. Due to the high angular momentum "barrier", the contribution from shortrange terms is marginal. In fact, since the contact terms at $\mathrm{N}^{4} \mathrm{LO}$ do not contribute for $L \geq 3$, there exists the unique opportunity to study the nucleon-nucleon force when it is controlled entirely by pion exchanges, which carry the signature of chiral symmetry. In short, states with $L \geq 3$ are a suitable ground to test the predictive power of chiral EFT. The LECs can be taken from $\pi N$ analysis, leaving no free parameters. Furthermore, the scattering phases in high angular momentum states are small, suggesting that a perturbative treatment would be appropriate. On the other hand, the latter is not suitable for the central partial waves, which require a non-perturbative approach to the solution of the Lippmann-Schwinger equation, with all its model (cutoff) dependence.

The perturbative $K$-matrix for $n p$ scattering is calculated as follows:

$$
K\left(\vec{p}^{\prime}, \vec{p}\right)=V_{1 \pi}^{(n p)}\left(\vec{p}^{\prime}, \vec{p}\right)+V_{2 \pi, \text { it }}^{(n p)}\left(\vec{p}^{\prime}, \vec{p}\right)+V_{3 \pi, \text { it }}^{(n p)}\left(\vec{p}^{\prime}, \vec{p}\right)+V\left(\vec{p}^{\prime}, \vec{p}\right)
$$

with $V_{1 \pi}^{(n p)}\left(\vec{p}^{\prime}, \vec{p}\right)$ as in Eq. 41 , and $V_{2 \pi, \text { it }}^{(n p)}\left(\vec{p}^{\prime}, \vec{p}\right)$ representing the once iterated $1 \mathrm{PE}$ given by

$$
V_{2 \pi, \text { it }}^{(n p)}\left(\vec{p}^{\prime}, \vec{p}\right)=\mathcal{P} \int \frac{d^{3} p^{\prime \prime}}{(2 \pi)^{3}} \frac{M_{N}^{2}}{E_{p^{\prime \prime}}} \frac{V_{1 \pi}^{(n p)}\left(\vec{p}^{\prime}, \vec{p}^{\prime \prime}\right) V_{1 \pi}^{(n p)}\left(\vec{p}^{\prime \prime}, \vec{p}\right)}{p^{2}-p^{\prime \prime 2}}
$$

where $\mathcal{P}$ denotes the principal value integral and $E_{p^{\prime \prime}}=\sqrt{M_{N}^{2}+p^{\prime \prime 2}}$. A calculation at LO includes only the first term on the right hand side of Eq. $(70), V_{1 \pi}^{(n p)}\left(\vec{p}^{\prime}, \vec{p}\right)$, while calculations at NLO or higher order also include the second term on the right hand side, $V_{2 \pi, \text { it }}^{(n p)}\left(\vec{p}^{\prime}, \vec{p}\right)$. At NNLO, the twice iterated 1PE should be included as well; and at 
Table 2. Low-energy constants as determined in Ref. 22. The sets 'GW' and ' $\mathrm{KH}$ ' are based upon the $\pi N$ partial wave analyses of Refs. [45] and [46, respectively. The $c_{i}$ appear in Eq. 18 and are in units of $\mathrm{GeV}^{-1}$. The $\bar{d}_{i}$ and $\bar{e}_{i}$ belong to $\widehat{\mathcal{L}}_{\pi N}^{(3)}$ and $\widehat{\mathcal{L}}_{\pi N}^{(4)}$ [cf. Eqs. 190 and 201$]$ and are in units of $\mathrm{GeV}^{-2}$ and $\mathrm{GeV}^{-3}$, respectively.

\begin{tabular}{crr}
\hline & GW & KH \\
\hline$c_{1}$ & -1.13 & -0.75 \\
$c_{2}$ & 3.69 & 3.49 \\
$c_{3}$ & -5.51 & -4.77 \\
$c_{4}$ & 3.71 & 3.34 \\
$\bar{d}_{1}+\bar{d}_{2}$ & 5.57 & 6.21 \\
$\bar{d}_{3}$ & -5.35 & -6.83 \\
$\bar{d}_{5}$ & 0.02 & 0.78 \\
$\bar{d}_{14}-\bar{d}_{15}$ & -10.26 & -12.02 \\
$\bar{e}_{14}$ & 1.75 & 1.52 \\
$\bar{e}_{15}$ & -5.80 & -10.41 \\
$\bar{e}_{16}$ & 1.76 & 6.08 \\
$\bar{e}_{17}$ & -0.58 & -0.37 \\
$\bar{e}_{18}$ & 0.96 & 3.26 \\
\hline
\end{tabular}

higher orders further iterations should be accounted for. However, we found that the difference between the once iterated $1 \mathrm{PE}$ and the infinitely iterated $1 \mathrm{PE}$ is so small that it could not be identified on the scale of our phase shift figures. For that reason, we omit iterations of $1 \mathrm{PE}$ beyond what is contained in $V_{2 \pi, \text { it }}^{(n p)}\left(\vec{p}^{\prime}, \vec{p}\right)$. Furthermore, $V_{3 \pi, \text { it }}^{(n p)}\left(\vec{p}^{\prime}, \vec{p}\right)$ stands for terms where irreducible $2 \mathrm{PE}$ is iterated with $1 \mathrm{PE}$.

Finally, the fourth term on the r.h.s. of Eq. (70), $V\left(\vec{p}^{\prime}, \vec{p}\right)$, stands for the irreducible multi-pion exchange contributions that occur at the order at which the calculation is conducted. In multi-pion exchanges, we use the average pion mass $m_{\pi}=138.039 \mathrm{MeV}$ and, thus, neglect the charge-dependence due to pion-mass splitting in irreducible multipion diagrams.

Throughout this paper, we use

$$
M_{N}=\frac{2 M_{p} M_{n}}{M_{p}+M_{n}}=938.9183 \mathrm{MeV} .
$$

Based upon relativistic kinematics, the CMS on-shell momentum $p$ is related to the kinetic energy of the incident neutron in the laboratory system ("Lab. Energy"), $T_{\text {lab }}$, by

$$
p^{2}=\frac{M_{p}^{2} T_{\mathrm{lab}}\left(T_{\mathrm{lab}}+2 M_{n}\right)}{\left(M_{p}+M_{n}\right)^{2}+2 T_{\mathrm{lab}} M_{p}},
$$

with $M_{p}=938.2720 \mathrm{MeV}$ and $M_{n}=939.5654 \mathrm{MeV}$ the proton and neutron masses, respectively.

The $K$-matrix, Eq. (70), is decomposed into partial waves following Ref. [42] and phase shifts are then calculated via

$$
\tan \delta_{L}\left(T_{\mathrm{lab}}\right)=-\frac{M_{N}^{2} p}{16 \pi^{2} E_{p}} p K_{L}(p, p) .
$$



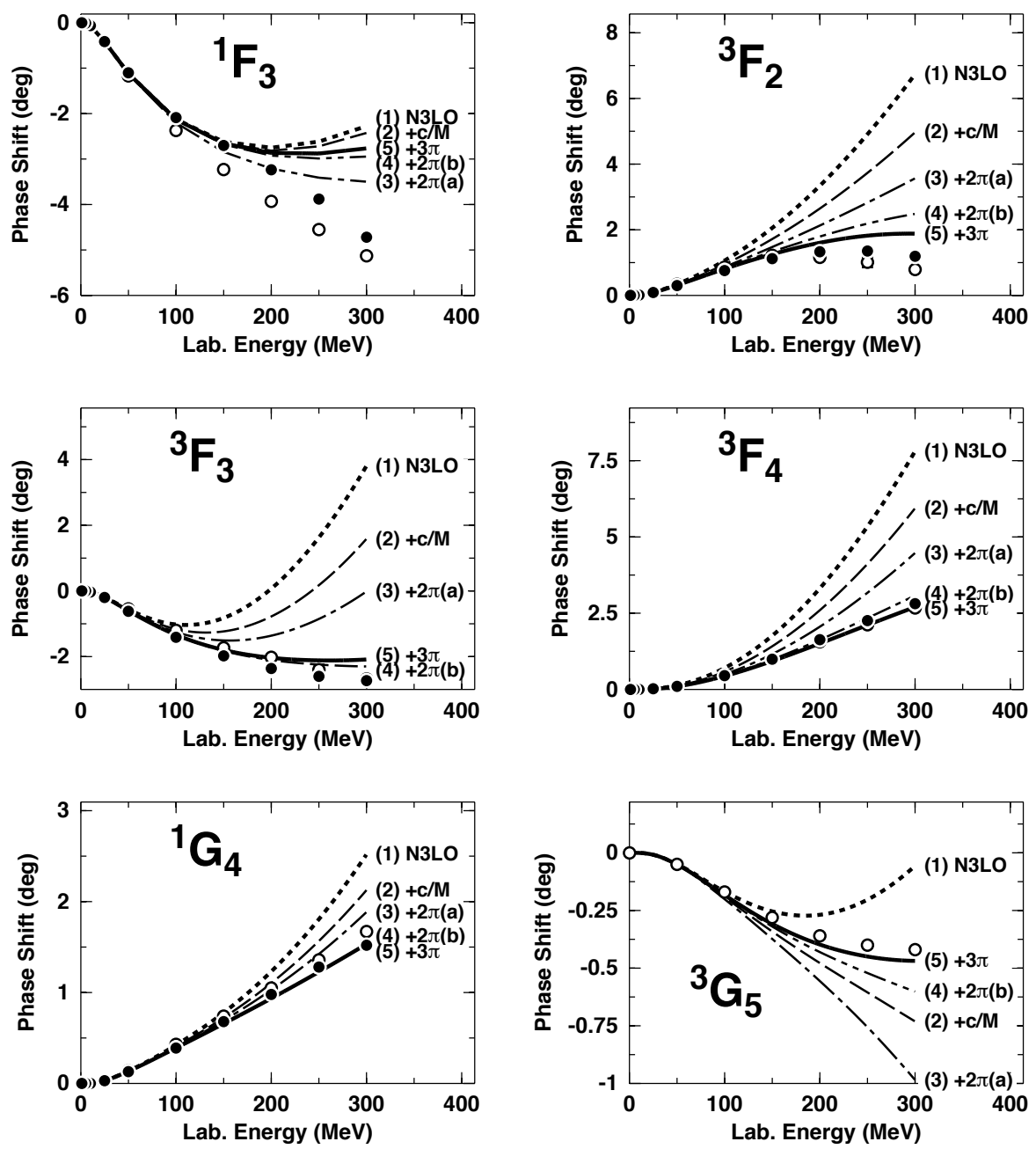

Figure 8. Effect of individual $\mathrm{N}^{4} \mathrm{LO}$ (fifth-order) contributions on the neutron-proton phase shifts of some selected peripheral partial waves. The individual contributions are added up successively in the order given in parenthesis next to each curve. Curve (1) is $\mathrm{N}^{3} \mathrm{LO}$ and curve (5) is the complete $\mathrm{N}^{4} \mathrm{LO}$. The KH LECs are used and $\tilde{\Lambda}=1.5$ $\mathrm{GeV}$. The filled and open circles represent the results from the Nijmegan multi-energy $n p$ phase-shift analysis [47] and the VPI/GW single-energy $n p$ analysis SM99 [48, respectively. (Figure reproduced from Ref. [29].)

For more details concerning the evaluation of phase shifts, including the case of coupled partial waves, see Ref. [43] or the appendix of [44].

Chiral symmetry establishes a link between the dynamics in the $\pi N$-system and the $N N$-system (through common low-energy constants). In order to check the consistency, we use the LECs for subleading $\pi N$-couplings as determined in analyses of low-energy elastic $\pi N$-scattering. Appropriate analyses for our purposes are contained in Refs. [22], where $\pi N$-scattering has been calculated at fourth order using the same power-counting of relativistic $1 / M_{N}$-corrections as in the present work. Reference [22] performed two fits, one to the GW [45] and one to the KH [46] partial wave analysis resulting in the two sets of LECs listed in Table 2 , 
The contributions up to $\mathrm{N}^{3} \mathrm{LO}$ and their impact on peripheral $N N$ scattering have been discussed and demonstrated in detail in Ref. [11] and, therefore, we will not repeat that demonstration here. But we will discuss the recent progress that has been made in the calculation of orders beyond $\mathrm{N}^{3} \mathrm{LO}$.

We start with the individual $\mathrm{N}^{4} \mathrm{LO}$ (fifth-order) contributions. For this purpose, we display in Fig. 8 phase shifts for six important peripheral partial waves, namely, ${ }^{1} F_{3}$, ${ }^{3} F_{2},{ }^{3} F_{3},{ }^{3} F_{4},{ }^{1} G_{4}$, and ${ }^{3} G_{5}$. In each frame, the following curves are shown:

(1) $\mathrm{N}^{3} \mathrm{LO}$.

(2) The previous curve plus the $c_{i} / M_{N}$ corrections (denoted by 'c/M'), Fig. 4(c).

(3) The previous curve plus the $\mathrm{N}^{4} \mathrm{LO} 2 \pi$-exchange two-loop contributions of class (a), Fig. 4(a).

(4) The previous curve plus the $\mathrm{N}^{4} \mathrm{LO} 2 \pi$ two-loop contributions of class (b), Fig. 4(b).

(5) The previous curve plus the $\mathrm{N}^{4} \mathrm{LO} 3 \pi$-exchange contributions, Fig. 5 .

In summary, the various curves add up successively the individual $\mathrm{N}^{4} \mathrm{LO}$ contributions in the order indicated in the curve labels. The last curve in this series, curve (5), is the full $\mathrm{N}^{4} \mathrm{LO}$ result. In these calculations, a SFR cutoff $\tilde{\Lambda}=1.5 \mathrm{GeV}$ is applied [cf. Eqs. (57) and (58)] and the KH LECs (cf. Table 2) are used.

From Fig. 8, we make the following observations. In triplet $F$-waves, the $c_{i} / M_{N}$ corrections as well as the $2 \mathrm{PE}$ two-loops, class (a) and (b), are all repulsive and of about the same strength. As a consequence, the problem of the excessive attraction, that $\mathrm{N}^{3} \mathrm{LO}$ is beset with, is overcome. A similar trend is seen in ${ }^{1} G_{4}$. An exception is ${ }^{1} F_{3}$, where the class (b) contribution is attractive leading to phase shifts above the data for energies higher than $150 \mathrm{MeV}$.

Now turning to the $\mathrm{N}^{4} \mathrm{LO} 3 \mathrm{PE}$ contributions [curve (5) in Fig. 8]: they are substantially smaller than the 2PE two-loop ones, in all peripheral partial waves. This can be interpreted as an indication of convergence with regard to the number of pions being exchanged between two nucleons - a trend that is very welcome. Further, note that the total $3 \mathrm{PE}$ contribution is a very comprehensive one, cf. Fig. 5. It is the sum of ten terms which, individually, can be fairly large. However, destructive interference between them leads to the small net result.

For all $F$ and $G$ waves (except ${ }^{1} F_{3}$ ), the final $\mathrm{N}^{4} \mathrm{LO}$ result is close to the empirical phase shifts. Notice that this includes also ${ }^{3} G_{5}$, which posed persistent problems at $\mathrm{N}^{3} \mathrm{LO}$ [49].

It is also of interest to know how predictions change with variations of $\tilde{\Lambda}$ within a reasonable range. We have, therefore, varied $\tilde{\Lambda}$ between 0.7 and $1.5 \mathrm{GeV}$ and show the predictions for all $F$ and $G$ waves in Figs. 9 and 10 , respectively, in terms of colored bands. It is seen that, at $\mathrm{N}^{3} \mathrm{LO}$, the variations of the predictions are very large and always too attractive while, at $\mathrm{N}^{4} \mathrm{LO}$, the variations are small and the predictions are close to the data or right on the data. Figs. 9 and 10 also include the lower orders (LO, NLO, and NNLO) such that a comparison of the relative size of the order-by-order contributions is possible. We observe that there is not much of a convergence, since 

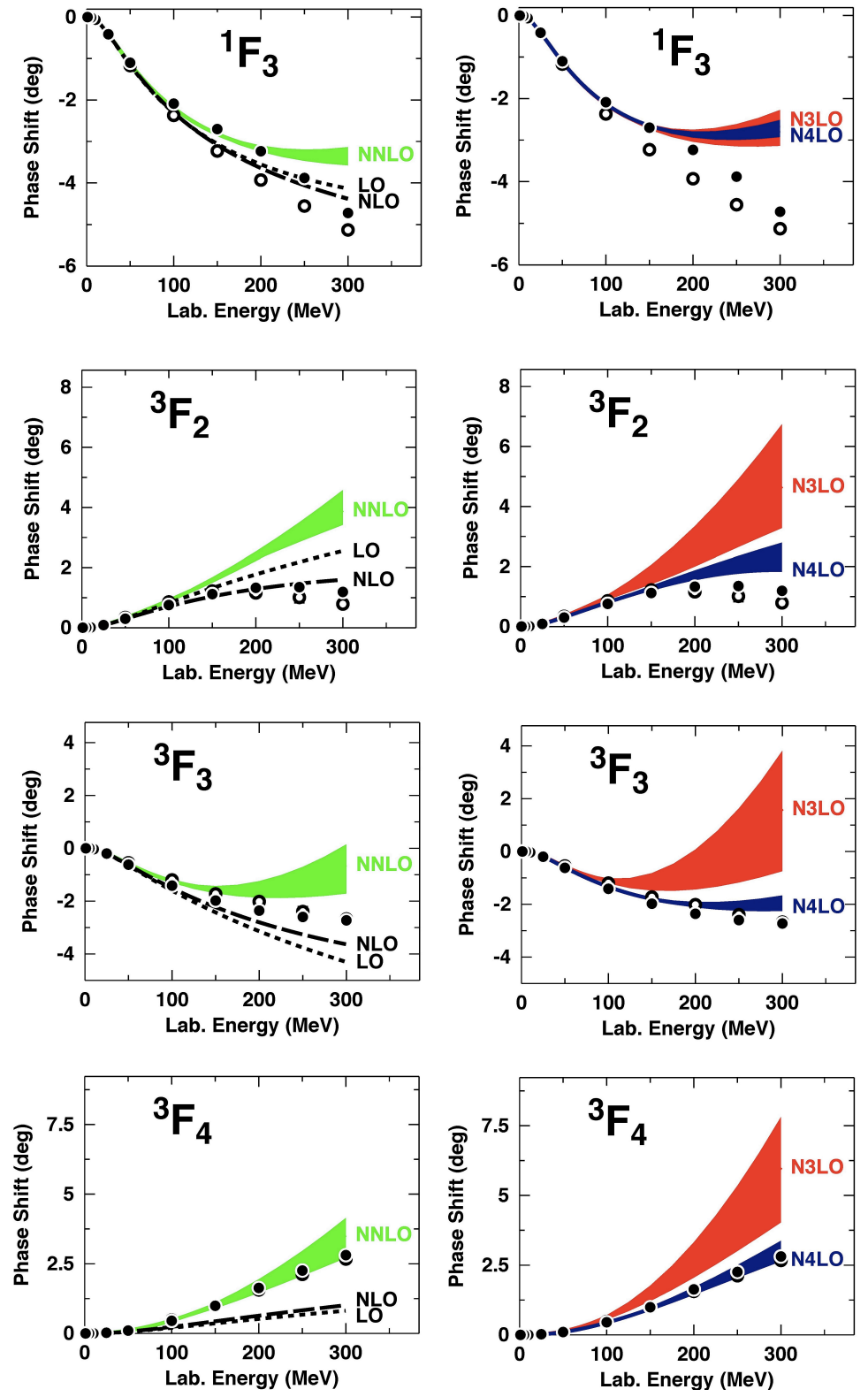

Figure 9. Phase-shifts of neutron-proton scattering at various orders up to $\mathrm{N}^{4} \mathrm{LO}$. The colored bands show the variation of the predictions when the SFR cutoff $\tilde{\Lambda}$ is changed over the range 0.7 to $1.5 \mathrm{GeV}$. The KH LECs are applied. Empirical phase shifts as in Fig. 8. (Figure reproduced from Ref. 29].)

obviously the magnitudes of the NNLO, $\mathrm{N}^{3} \mathrm{LO}$, and $\mathrm{N}^{4} \mathrm{LO}$ contributions are about the same.

To obtain more insight into the convergence issue, we need to proceed to the next order, which is $\mathrm{N}^{5} \mathrm{LO}$. As shown in Figs. 6 and 7, the sixth-order corrections consist of several contributions. As in the case of $\mathrm{N}^{4} \mathrm{LO}$, we will first show how the individual $\mathrm{N}^{5} \mathrm{LO}$ contributions impact $N N$-phase-shifts in peripheral waves. In Fig. 11, we display phase-shifts for two peripheral partial waves, namely, ${ }^{1} G_{4}$, and ${ }^{3} G_{5}$. The following 

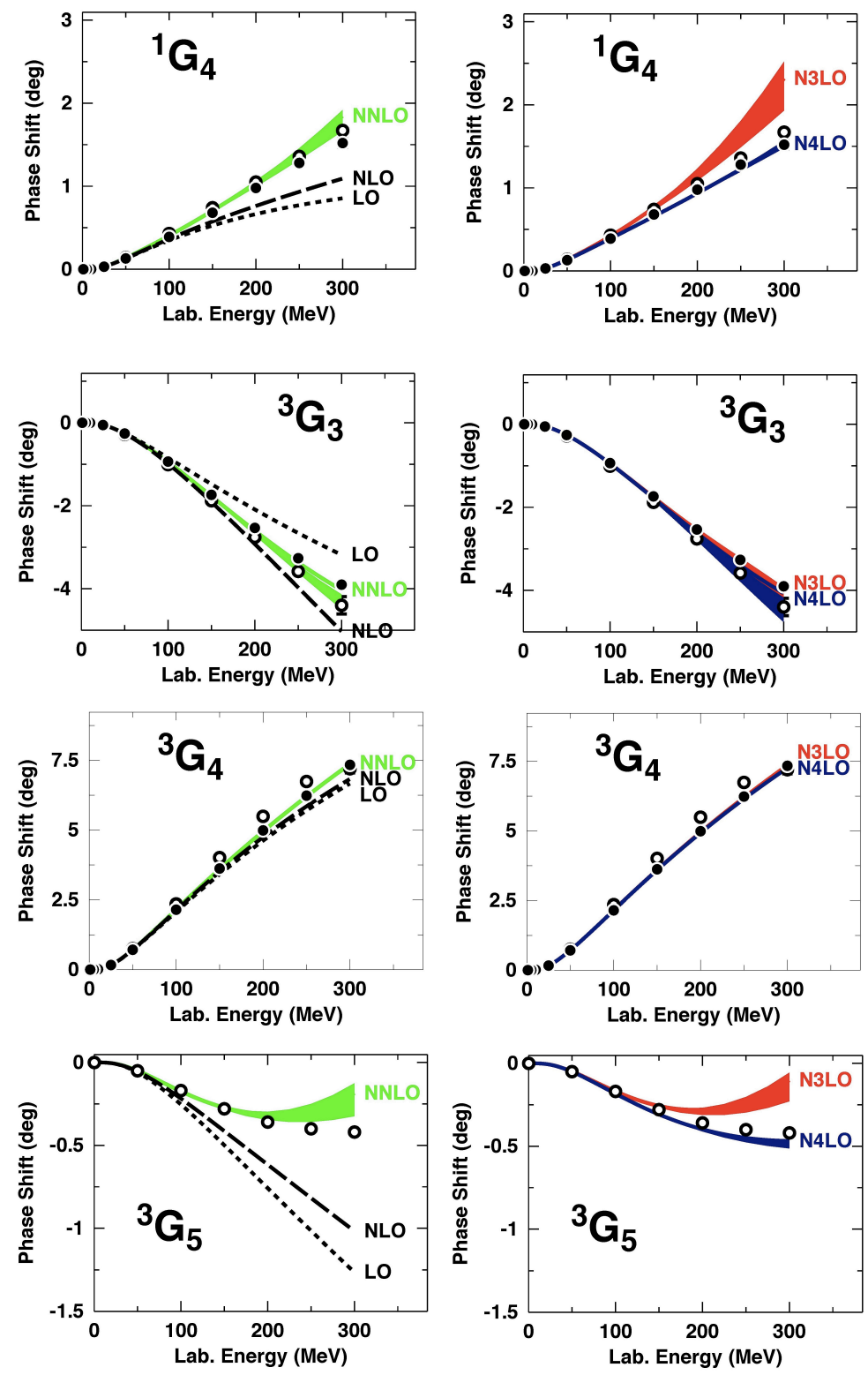

Figure 10. Same as Fig. 9, but for $G$-waves. (Figure reproduced from Ref. 29].)

curves are shown:

(1) $\mathrm{N}^{4} \mathrm{LO}$.

(2) The previous curve plus the $\mathrm{N}^{5} \mathrm{LO} 2 \pi$-exchange contributions of class (a), Fig. 6(a).

(3) The previous curve plus the $\mathrm{N}^{5} \mathrm{LO} 2 \pi$-exchange contributions of class (b), Fig. 6(b).

(4) The previous curve plus the $\mathrm{N}^{5} \mathrm{LO} 3 \pi$-exchange contributions of class (a), Fig. 7(a).

(5) The previous curve plus the $\mathrm{N}^{5} \mathrm{LO} 3 \pi$-exchange contributions of class (b), Fig. 7(b).

(6) The previous curve plus the $1 / M_{N}^{2}$-corrections (denoted by ' $1 / \mathrm{M} 2$ ') [41].

The last curve in this series, curve (6), includes all $\mathrm{N}^{5} \mathrm{LO}$ contributions calculated in 

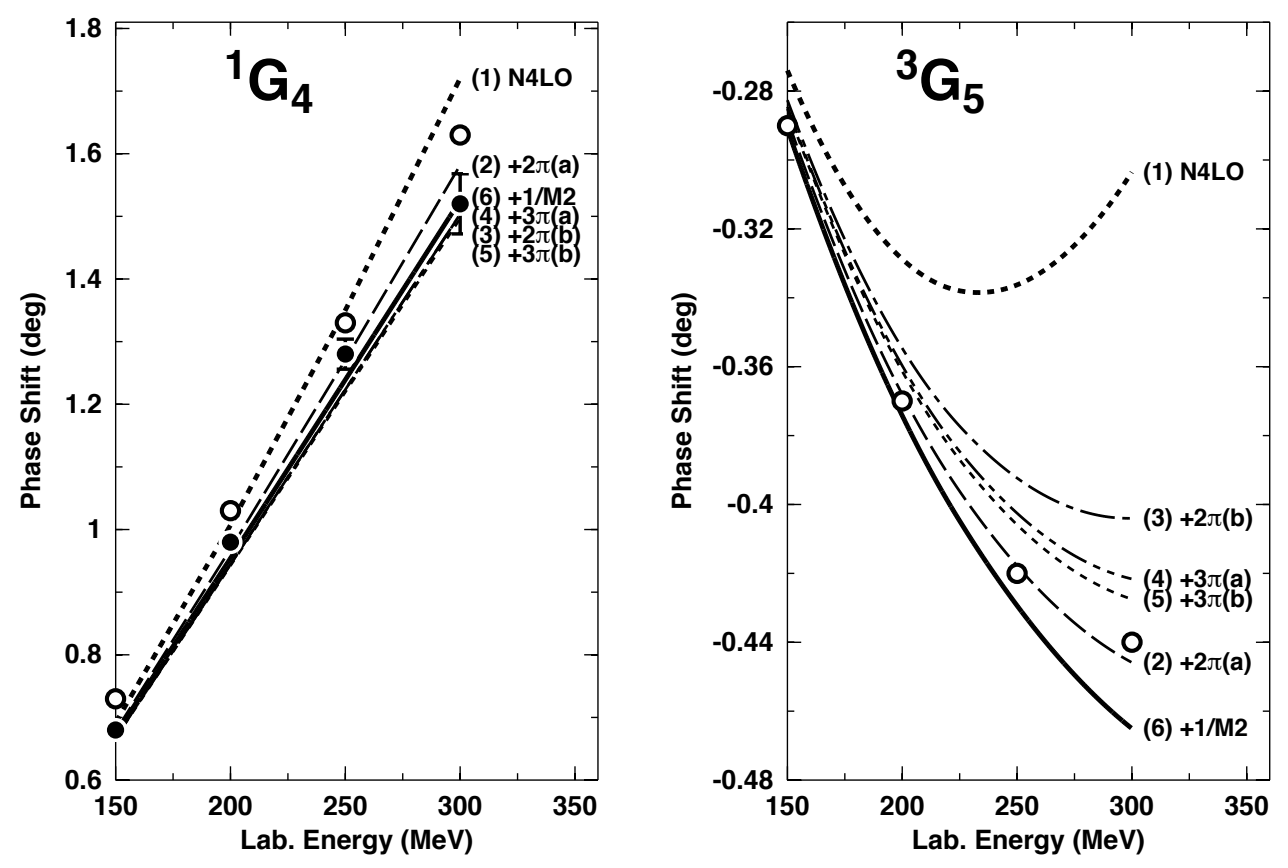

Figure 11. Effect of individual $\mathrm{N}^{5} \mathrm{LO}$ (sixth-order) contributions on the neutronproton phase shifts of two $G$-waves. The individual contributions are added up successively in the order given in parentheses next to each curve. Curve (1) is $\mathrm{N}^{4} \mathrm{LO}$ and curve (6) contains all $\mathrm{N}^{5} \mathrm{LO}$ contributions calculated in Ref. 32. A SFR cutoff $\tilde{\Lambda}=800 \mathrm{MeV}$ is applied and the GW LECs are used. The filled and open circles represent the results from the Nijmegen multi-energy $n p$ phase-shift analysis 47] and the GW np-analysis SP07 [50, respectively. (Figure reproduced from Ref. [32].)

Ref. [32]. For all curves of this figure, a SFR cutoff $\tilde{\Lambda}=800 \mathrm{MeV}$ [cf. Eqs. (57) and (58)] is employed and the GW (cf. Table 2) LECs are used.

From Fig. 11, we see that the two-loop $2 \pi$-exchange class (a), Fig. 6(a), generates a strong repulsive central force, while the spin-spin and tensor forces provided by this class are negligible. The fact that this class produces a relatively large contribution is not unexpected, since it is proportional to $c_{i}^{2}$. The $2 \pi$-exchange contribution class (b), Fig. 6(b), creates a moderately repulsive central force as seen by its effect on ${ }^{1} G_{4}$ and a noticeable tensor force as the impact on ${ }^{3} G_{5}$ demonstrates. The $3 \pi$-exchange class (a), Fig. 7(a), is negligible in ${ }^{1} G_{4}$, but noticeable in ${ }^{3} G_{5}$ and, therefore, it should not be neglected. This contribution is proportional to $c_{i}^{2}$, which suggests a non-negligible size but it is typically smaller than the corresponding $2 \pi$-exchange contribution class (a). The $3 \pi$-exchange class (b) contribution, Fig. 7(b), turns out to be negligible [see the difference between curve (4) and (5) in Fig. 11. This may not be unexpected since it is a three-loop contribution with only leading-order vertices. Finally the relativistic $1 / M_{N^{-}}^{2}$ corrections to the leading $2 \pi$-exchange [41] have a small but non-negligible impact, particularly in ${ }^{3} G_{5}$.

The $\mathrm{N}^{5} \mathrm{LO}$ predictions for all $G$ and $H$ waves are displayed in Fig. 12 in terms of colored bands that are generated by varying the SFR cutoff $\tilde{\Lambda}$ [cf. Eqs. (57) and 
(58)] between 700 and $900 \mathrm{MeV}$. The figure clearly reveals again that, at $\mathrm{N}^{3} \mathrm{LO}$, the predictions are, in general, too attractive. As discussed, the $\mathrm{N}^{4} \mathrm{LO}$ contribution, essentially, compensates this attractive surplus. $\mathrm{N}^{5} \mathrm{LO}$ then adds additional repulsion bringing the final prediction right onto the data (i.e. empirical phase-shifts). Moreover, the $\mathrm{N}^{5} \mathrm{LO}$ contribution is, in general, substantially smaller than the one at $\mathrm{N}^{4} \mathrm{LO}$, thus, showing a signature of convergence of the chiral expansion.

To summarize, we present in Fig. 13 a comparison between all orders from LO to $\mathrm{N}^{5} \mathrm{LO}$. Note that the difference between the LO prediction (one-pion-exchange, dotted line) and the data (filled and open circles) is to be provided by two- and three-pion exchanges, i.e. the intermediate-range part of the nuclear force. How well that is accomplished is a crucial test for any theory of nuclear forces. NLO produces only a small contribution, but $\mathrm{N}^{2} \mathrm{LO}$ creates substantial intermediate-range attraction (most clearly seen in ${ }^{1} G_{4},{ }^{3} G_{5}$, and ${ }^{3} H_{6}$ ). In fact, $\mathrm{N}^{2} \mathrm{LO}$ is the largest contribution among all orders. This is due to the one-loop $2 \pi$-exchange triangle diagram which involves one $\pi \pi N N$-contact vertex proportional to $c_{i}$. This vertex represents correlated $2 \mathrm{PE}$ as well as intermediate $\Delta(1232)$-isobar excitation. It is well-known from the traditional meson theory of nuclear forces [6, 7] that these two features are crucial for a realistic and quantitative $2 \mathrm{PE}$ model. Consequently, the one-loop $2 \pi$-exchange at $\mathrm{N}^{2} \mathrm{LO}$ is attractive and assumes a realistic size describing the intermediate-range attraction of the nuclear force almost correctly. At $\mathrm{N}^{3} \mathrm{LO}$, more one-loop $2 \mathrm{PE}$ is added by the bubble diagram with two $c_{i}$-vertices, a contribution that seems to overestimate the attraction. This attractive surplus is then compensated by the prevailingly repulsive two-loop $2 \pi$ - and $3 \pi$-exchanges that occur at $\mathrm{N}^{4} \mathrm{LO}$ and $\mathrm{N}^{5} \mathrm{LO}$.

In this context, it is worth noting that also in conventional meson theory [7] the

one-loop models for the 2PE contribution always show some excess of attraction (cf. Figs. 7-9 of Ref. [49]). The same is true for the dispersion theoretic approach pursued by the Paris group [6]. In conventional meson theory, the surplus attraction is reduced by heavy-meson exchange ( $\rho$ - and $\omega$-exchange) which, however, has no place in chiral effective field theory (as a finite-range contribution). Instead, in the latter approach, two-loop $2 \pi$ - and $3 \pi$-exchanges provide the corrective action.

\section{Constructing complete chiral $N N$ potentials}

Previously, we addressed the long- and medium-range parts of the nuclear interaction, which involve pion-exchange contributions. Because of their long-range nature, these terms control partial waves with high values of $L$ and are governed by chiral symmetry. Of course, to obtain quantitative predictions of low-energy $N N$ scattering observables or nuclear properties, all partial waves must be described realistically, in particular the most central ones $(L \leq 2)$, which carry information on the dynamics at short range. The latter will be our next concern. 

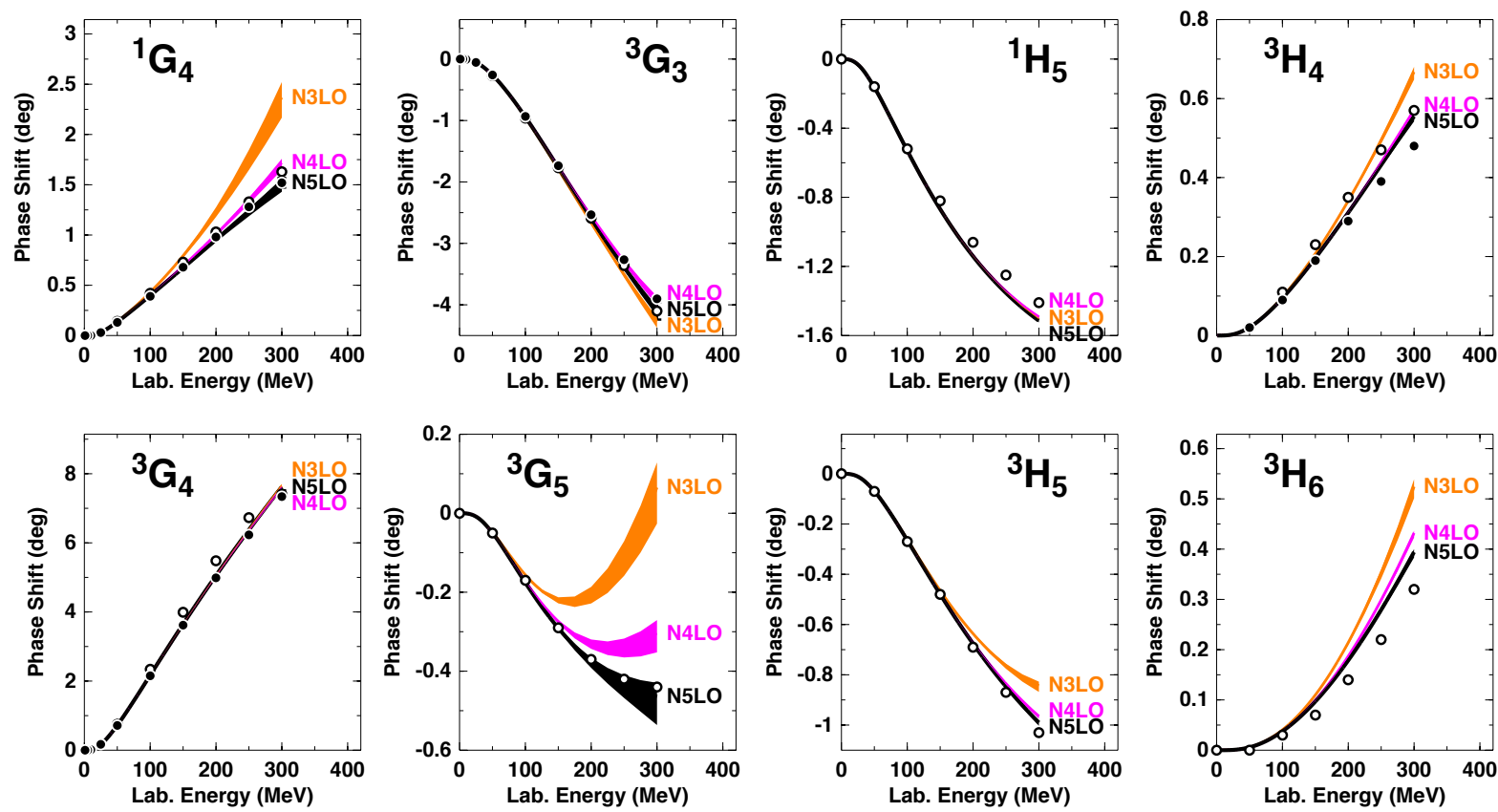

Figure 12. Phase-shifts of neutron-proton scattering in $G$ and $H$ waves at $\mathrm{N}^{3} \mathrm{LO}$, $\mathrm{N}^{4} \mathrm{LO}$, and $\mathrm{N}^{5} \mathrm{LO}$. The colored bands show the variations of the predictions when the SFR cutoff $\tilde{\Lambda}$ is changed over the range 700 to $900 \mathrm{MeV}$. The GW LECs are applied.

Empirical phase shifts are as in Fig. 11. (Figure reproduced from Ref. 32.)
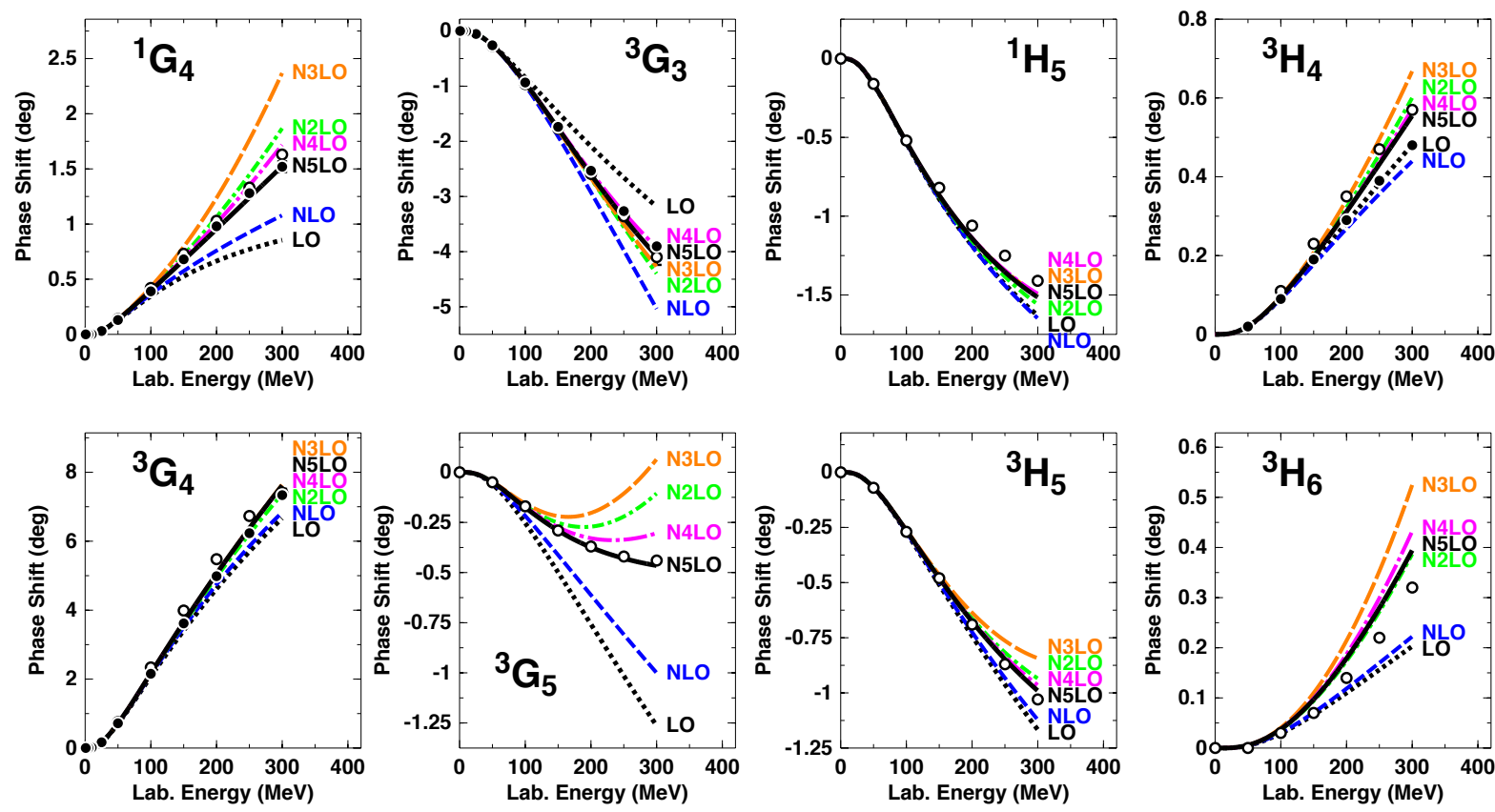

Figure 13. Phase-shifts of neutron-proton scattering in $G$ and $H$ waves at all orders from LO to $\mathrm{N}^{5} \mathrm{LO}$. A SFR cutoff $\tilde{\Lambda}=800 \mathrm{MeV}$ is used and the GW LECs are applied. Empirical phase shifts are as in Fig. 11. (Figure reproduced from Ref. 32.) 


\subsection{NN contact terms}

It has been known for a long time that the bulk of the short-distance behavior of the nuclear force can be explained with the introduction of heavy bosons, e.g. the $\omega(782)$. Applying Fourier transformation to the propagator of the meson,

$$
\int \frac{d^{3} q}{(2 \pi)^{3}} \frac{e^{i \vec{q} \cdot \vec{r}}}{m_{\omega}^{2}+\vec{q}^{2}}=\frac{1}{4 \pi} \frac{e^{-m_{\omega} r}}{r},
$$

provides a qualitative description of the $N N$ force at short range.

Since ChPT is an expansion valid for small values of the momentum, mesons such as the $\rho(770)$ or the $\omega(782)$ are outside its resolution power (notice that $\Lambda_{\chi} \approx m_{\rho, \omega}$ ). However, the propagator of the heavy boson under consideration can be handled with an expansion,

$$
\frac{1}{m_{\omega}^{2}+Q^{2}} \approx \frac{1}{m_{\omega}^{2}}\left(1-\frac{Q^{2}}{m_{\omega}^{2}}+\frac{Q^{4}}{m_{\omega}^{4}}-+\ldots\right) .
$$

One may then approach the short-range $N N$ interaction guided by the expansion above, namely as a power series in $Q / m_{\omega}$. This is the origin of the contributions referred to as contact terms.

Contact terms play an important role in renormalization. Contributions involving the exchange of more than one pion entail loop integrals, which produce polynomial terms whose coefficients can be divergent or scale dependent (cf. Appendix B of Ref. [11]). Contact terms are then crucial to remove those divergences or scale dependences and so they act as "counter terms".

Our procedure will involve partial-wave expansion of terms polynomial in $Q$, where $Q$ stands for the momentum transfer between the two nucleons, $q$, or their average momentum $k$ [see below Eq. (39) for their definitions]. In any case, for even $\nu$,

$$
Q^{\nu}=f_{\frac{\nu}{2}}(\cos \theta)
$$

where $f_{m}$ stands for a polynomial of degree $m$ and $\theta$ is the scattering angle in the center-of-mass system. When expanding $Q^{\nu}$ in partial waves, we encounter the integral

$$
I_{L}^{(\nu)}=\int_{-1}^{+1} Q^{\nu} P_{L}(\cos \theta) d \cos \theta=\int_{-1}^{+1} f_{\frac{\nu}{2}}(\cos \theta) P_{L}(\cos \theta) d \cos \theta,
$$

where $L$ is the orbital angular momentum and $P_{L}$ is a Legendre polynomial. Since Legendre polynomials are orthogonal,

$$
I_{L}^{(\nu)}=0 \quad \text { for } \quad L>\frac{\nu}{2} .
$$

Therefore, we can see that contact terms of a particular order can only contribute up to some partial wave.

Parity conservation forbids the presence of odd powers of $Q$. Therefore, the contact interaction can be formally written as

$$
V_{\mathrm{ct}}=V_{\mathrm{ct}}^{(0)}+V_{\mathrm{ct}}^{(2)}+V_{\mathrm{ct}}^{(4)}+V_{\mathrm{ct}}^{(6)}+\ldots,
$$

with the superscript indicating a given order.

Next, we display the contact $N N$ potentials at each order as they emerge from the Lagrangians shown at the end of Section 2.2. 
6.1.1. Zeroth order (LO) From the Lagrangian $\widehat{\mathcal{L}}_{N N}^{(0)}$, Eq. 23) (which is part of $\widehat{\mathcal{L}}^{\Delta=0}$, Eq. (17)), we can generate the contact potential

$$
V_{\mathrm{ct}}^{(0)}\left(\overrightarrow{p^{\prime}}, \vec{p}\right)=C_{S}+C_{T} \vec{\sigma}_{1} \cdot \vec{\sigma}_{2}
$$

whose partial-wave contributions are

$$
\begin{aligned}
& V_{\mathrm{ct}}^{(0)}\left({ }^{1} S_{0}\right)=\widetilde{C}_{{ }_{S_{0}}}=4 \pi\left(C_{S}-3 C_{T}\right) \\
& V_{\mathrm{ct}}^{(0)}\left({ }^{3} S_{1}\right)=\widetilde{C}_{{ }^{3} S_{1}}=4 \pi\left(C_{S}+C_{T}\right) .
\end{aligned}
$$

6.1.2. Second order (NLO) For this, we refer to $\widehat{\mathcal{L}}_{N N}^{(2)}$, Eq. 24) (part of $\widehat{\mathcal{L}}^{\Delta=2}$, Eq. (19)). We now have

$$
\begin{aligned}
V_{\mathrm{ct}}^{(2)}\left(\overrightarrow{p^{\prime}}, \vec{p}\right) & =C_{1} q^{2}+C_{2} k^{2} \\
& +\left(C_{3} q^{2}+C_{4} k^{2}\right) \vec{\sigma}_{1} \cdot \vec{\sigma}_{2} \\
& +C_{5}(-i \vec{S} \cdot(\vec{q} \times \vec{k})) \\
& +C_{6}\left(\vec{\sigma}_{1} \cdot \vec{q}\right)\left(\vec{\sigma}_{2} \cdot \vec{q}\right) \\
& +C_{7}\left(\vec{\sigma}_{1} \cdot \vec{k}\right)\left(\vec{\sigma}_{2} \cdot \vec{k}\right) .
\end{aligned}
$$

Notice that the constants $C_{i}$ which appear in these expressions are related to the coefficients $C_{i}^{\prime}$ present in the Lagrangian $\widehat{\mathcal{L}}_{N N}^{(2)}$, Eq. [24), see Refs. [23, 51] for details (not relevant for us at this point).

One way to partial-wave decompose the potential above is the method proposed by Erkelenz, Alzetta, and Holinde [42]. One obtains

$$
\begin{array}{ll}
V_{\mathrm{ct}}^{(2)}\left({ }^{1} S_{0}\right) & =C_{{ } S_{0}}\left(p^{2}+p^{2}\right) \\
V_{\mathrm{ct}}^{(2)}\left({ }^{3} P_{0}\right) & =C^{3} P_{0} p p^{\prime} \\
V_{\mathrm{ct}}^{(2)}\left({ }^{1} P_{1}\right) & =C_{{ }_{1} P_{1}} p p^{\prime} \\
V_{\mathrm{ct}}^{(2)}\left({ }^{3} P_{1}\right) & =C P_{1} p p^{\prime} \\
V_{\mathrm{ct}}^{(2)}\left({ }^{3} S_{1}\right) & =C{ }_{S_{1}}\left(p^{2}+p^{\prime 2}\right) \\
V_{\mathrm{ct}}^{(2)}\left({ }^{3} S_{1}-{ }^{3} D_{1}\right) & =C^{3} S_{1}-{ }^{3} D_{1} p^{2} \\
V_{\mathrm{ct}}^{(2)}\left({ }^{3} D_{1}-{ }^{3} S_{1}\right) & =C_{{ }^{3} S_{1}-{ }^{3} D_{1} p^{\prime 2}} \\
V_{\mathrm{ct}}^{(2)}\left({ }^{3} P_{2}\right) & =C_{3} P_{2} p p^{\prime}
\end{array}
$$

with

$$
\begin{array}{ll}
C_{1 S_{0}} & =4 \pi\left(C_{1}+\frac{1}{4} C_{2}-3 C_{3}-\frac{3}{4} C_{4}-C_{6}-\frac{1}{4} C_{7}\right) \\
C^{3} P_{0} & =4 \pi\left(-\frac{2}{3} C_{1}+\frac{1}{6} C_{2}-\frac{2}{3} C_{3}+\frac{1}{6} C_{4}-\frac{2}{3} C_{5}+2 C_{6}-\frac{1}{2} C_{7}\right) \\
C^{{ }_{1} P_{1}} & =4 \pi\left(-\frac{2}{3} C_{1}+\frac{1}{6} C_{2}+2 C_{3}-\frac{1}{2} C_{4}+\frac{2}{3} C_{6}-\frac{1}{6} C_{7}\right) \\
C^{3} P_{1} & =4 \pi\left(-\frac{2}{3} C_{1}+\frac{1}{6} C_{2}-\frac{2}{3} C_{3}+\frac{1}{6} C_{4}-\frac{1}{3} C_{5}-\frac{4}{3} C_{6}+\frac{1}{3} C_{7}\right) \\
C^{{ } S_{1}} & =4 \pi\left(C_{1}+\frac{1}{4} C_{2}+C_{3}+\frac{1}{4} C_{4}+\frac{1}{3} C_{6}+\frac{1}{12} C_{7}\right)
\end{array}
$$


Chiral EFT based nuclear forces

$$
\begin{aligned}
& C_{{ }^{3} S_{1}-{ }^{3} D_{1}}=4 \pi\left(-\frac{2 \sqrt{2}}{3} C_{6}-\frac{\sqrt{2}}{6} C_{7}\right) \\
& C_{{ }_{P_{2}}}=4 \pi\left(-\frac{2}{3} C_{1}+\frac{1}{6} C_{2}-\frac{2}{3} C_{3}+\frac{1}{6} C_{4}+\frac{1}{3} C_{5}\right) .
\end{aligned}
$$

6.1.3. Fourth order $\left(N^{3} L O\right)$ The contact potential of order four reads

$$
\begin{aligned}
V_{\mathrm{ct}}^{(4)}\left(\overrightarrow{p^{\prime}}, \vec{p}\right) & =D_{1} q^{4}+D_{2} k^{4}+D_{3} q^{2} k^{2}+D_{4}(\vec{q} \times \vec{k})^{2} \\
& +\left(D_{5} q^{4}+D_{6} k^{4}+D_{7} q^{2} k^{2}+D_{8}(\vec{q} \times \vec{k})^{2}\right) \vec{\sigma}_{1} \cdot \vec{\sigma}_{2} \\
& +\left(D_{9} q^{2}+D_{10} k^{2}\right)(-i \vec{S} \cdot(\vec{q} \times \vec{k})) \\
& +\left(D_{11} q^{2}+D_{12} k^{2}\right)\left(\vec{\sigma}_{1} \cdot \vec{q}\right)\left(\vec{\sigma}_{2} \cdot \vec{q}\right) \\
& +\left(D_{13} q^{2}+D_{14} k^{2}\right)\left(\vec{\sigma}_{1} \cdot \vec{k}\right)\left(\vec{\sigma}_{2} \cdot \vec{k}\right) \\
& +D_{15}\left(\vec{\sigma}_{1} \cdot(\vec{q} \times \vec{k}) \vec{\sigma}_{2} \cdot(\vec{q} \times \vec{k})\right) .
\end{aligned}
$$

The corresponding partial-wave expressions at this order can be found in Appendix E of Ref. [11].

6.1.4. Sixth order $\left(N^{5} L O\right)$ At sixth order, 26 new contact terms appear, bringing the total number to 50 . These terms as well as their partial-wave decomposition have been worked out in Ref. [33]. So far, these terms have not been used in the construction of $N N$ potentials.

\subsection{Definition of $N N$ potential}

At this point, we have all the "ingredients" required to describe the well-known phenomenology of the nuclear force at long, medium, and short distances. When approaching the most central waves, though, we are faced with one more hurdle. As is known from the most elementary nuclear physics, the $N N$ system at $L=0$ admits a bound state, the weakly-bound deuteron, and large scattering lengths, which do not allow for a perturbative treatment. Moreover, unlike what happens with $\pi-\pi$ and $\pi-N$ in the chiral limit, the interaction of nucleons does not vanish when $Q \rightarrow 0$. As argued by Weinberg [9], intermediate states with only nucleons are responsible for the large increase of the scattering amplitude commonly referred to as "infrared enhancement". A way to circumvent this problem, as suggested by Weinberg, is to calculate the $N N$ potential perturbatively and then to apply it in a scattering equation to obtain the $N N$ amplitude. This is the strategy we will adopt.

The pion-exchange parts of the $N N$ potential were spelled out in Eqs. (33)-(38). To obtain the complete potential, one just has to add to this the contact terms listed in Eq. (80). Thus, one has to do the following extensions to some of the Eqs. (33)-(38):

$$
\begin{aligned}
& V_{\mathrm{LO}} \longmapsto V_{\mathrm{LO}}+V_{\mathrm{ct}}^{(0)} \\
& V_{\mathrm{NLO}} \longmapsto V_{\mathrm{NLO}}+V_{\mathrm{ct}}^{(2)} \\
& V_{\mathrm{N} 3 \mathrm{LO}} \longmapsto V_{\mathrm{N} 3 \mathrm{LO}}+V_{\mathrm{ct}}^{(4)}
\end{aligned}
$$


Chiral EFT based nuclear forces

$$
V_{\mathrm{N} 5 \mathrm{LO}} \longmapsto V_{\mathrm{N} 5 \mathrm{LO}}+V_{\mathrm{ct}}^{(6)}
$$

and no changes to $V_{\mathrm{NNLO}}$ and $V_{\mathrm{N} 4 \mathrm{LO}}$.

The potential $V$ as derived in previous sections is, in principal, an invariant amplitude and, thus, satisfies a relativistic scattering equation, for which we choose the BbS equation [11], which reads explicitly,

$$
T\left(\vec{p}^{\prime}, \vec{p}\right)=V\left(\vec{p}^{\prime}, \vec{p}\right)+\int \frac{d^{3} p^{\prime \prime}}{(2 \pi)^{3}} V\left(\vec{p}^{\prime}, \vec{p}^{\prime \prime}\right) \frac{M_{N}^{2}}{E_{p^{\prime \prime}}} \frac{1}{p^{2}-p^{\prime 2}+i \epsilon} T\left(\vec{p}^{\prime \prime}, \vec{p}\right)
$$

with $E_{p^{\prime \prime}} \equiv \sqrt{M_{N}^{2}+p^{\prime \prime 2}}$. The use of a relativistic equation implies that relativistic corrections are already included to all orders (no additional corrections are needed when increasing the EFT order).

If we define

$$
\widehat{V}\left(\vec{p}^{\prime}, \vec{p}\right) \equiv \frac{1}{(2 \pi)^{3}} \sqrt{\frac{M_{N}}{E_{p^{\prime}}}} V\left(\vec{p}^{\prime}, \vec{p}\right) \sqrt{\frac{M_{N}}{E_{p}}}
$$

and

$$
\widehat{T}\left(\vec{p}^{\prime}, \vec{p}\right) \equiv \frac{1}{(2 \pi)^{3}} \sqrt{\frac{M_{N}}{E_{p^{\prime}}}} T\left(\vec{p}^{\prime}, \vec{p}\right) \sqrt{\frac{M_{N}}{E_{p}}},
$$

where the factor $1 /(2 \pi)^{3}$ is simply a convenient choice, the BbS equation assumes the form of the nonrelativistic Lippmann-Schwinger (LS) equation,

$$
\widehat{T}\left(\vec{p}^{\prime}, \vec{p}\right)=\widehat{V}\left(\vec{p}^{\prime}, \vec{p}\right)+\int d^{3} p^{\prime \prime} \widehat{V}\left(\vec{p}^{\prime}, \vec{p}^{\prime \prime}\right) \frac{M_{N}}{p^{2}-p^{\prime \prime 2}+i \epsilon} \widehat{T}\left(\vec{p}^{\prime \prime}, \vec{p}\right) .
$$

Since $\widehat{V}$ satisfies Eq. 94), it may be regarded as a nonrelativistic potential. By the same arguments, $\widehat{T}$ may be regarded as the nonrelativistic T-matrix. All technical aspects associated with the solution of the LS equation can be found in Appendix A of Ref. [44], including specific formulas for the $n p$ and $p p$ phase shifts. Additional details concerning the relevant operators and their decompositions are given in section 4 of Ref. [42]. Finally, computational methods to solve the LS equation are found in Ref. [43.

\subsection{Regularization and non-perturbative renormalization}

Iteration of $\widehat{V}$ in the LS equation, Eq. (94), requires cutting $\widehat{V}$ off for high momenta to avoid infinities. This is consistent with the fact that ChPT is a low-momentum expansion which is valid only for momenta $Q \ll \Lambda_{\chi} \approx 1 \mathrm{GeV}$. Therefore, the potential $\widehat{V}$ is multiplied with the regulator function $f\left(p^{\prime}, p\right)$,

$$
\widehat{V}\left(\vec{p}^{\prime}, \vec{p}\right) \longmapsto \widehat{V}\left(\vec{p}^{\prime}, \vec{p}\right) f\left(p^{\prime}, p\right)
$$

with

$$
f\left(p^{\prime}, p\right)=\exp \left[-\left(p^{\prime} / \Lambda\right)^{2 n}-(p / \Lambda)^{2 n}\right]
$$

such that

$$
\widehat{V}\left(\vec{p}^{\prime}, \vec{p}\right) f\left(p^{\prime}, p\right) \approx \widehat{V}\left(\vec{p}^{\prime}, \vec{p}\right)\left\{1-\left[\left(\frac{p^{\prime}}{\Lambda}\right)^{2 n}+\left(\frac{p}{\Lambda}\right)^{2 n}\right]+\ldots\right\}
$$



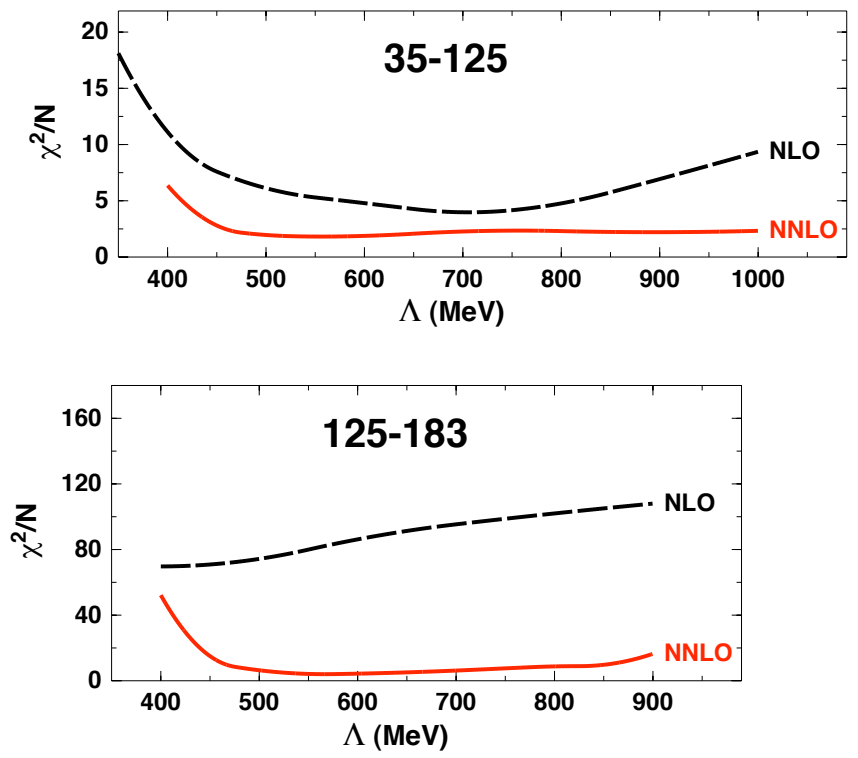

Figure 14. $\chi^{2} /$ datum for the reproduction of the $n p$ data in the energy range 35 $125 \mathrm{MeV}$ (upper frame) and 125-183 MeV (lower frame) as a function of the cutoff parameter $\Lambda$ of the regulator function Eq. (96). The (black) dashed curves show the $\chi^{2} /$ datum achieved with $n p$ potentials constructed at order NLO and the (red) solid curves are for NNLO. (Figure reproduced from Ref. [53.)

Typical choices for the cutoff parameter $\Lambda$ that appears in the regulator are $\Lambda \approx$ $0.5 \mathrm{GeV}<\Lambda_{\chi} \approx 1 \mathrm{GeV}$. At $\mathrm{N}^{3} \mathrm{LO}$ and $\mathrm{N}^{4} \mathrm{LO}$, an appropriate choice for $n$ is three.

We display Eq. (97) to demonstrate that the exponential cutoff may not impact the order at which we are working. If $n$ is sufficiently large, the regulating function generates terms beyond the given order. Under the assumption of a reasonable convergence of the chiral expansion, these terms are sufficiently small not to impact the accuracy at the present order. But note that the form as given in Eq. (96), and not its expansion Eq. (97), is used in actual calculations. We also mention in this context that the squareroot factors in Eqs. (92,93) are not expanded, as their full structure ensures consistency with relativistic elastic unitarity.

It is pretty obvious that results for the $T$-matrix may depend sensitively on the regulator and its cutoff parameter. The removal of such regulator dependence is known as renormalization. Proper renormalization of the chiral $N N$ interaction is a controversial issue, see Section 4.5 of Ref. [11] for a more comprehensive discussion.

For a successful EFT (in its domain of validity), one must be able to claim independence of the predictions on the regulator. Also, truncation error must decrease as we go to higher and higher orders. These are precisely the goals of renormalization.

Lepage 52] has stressed that the cutoff independence should be examined for cutoffs below the hard scale and not beyond. Ranges of cutoff independence within the theoretical error are to be identified using Lepage plots [52]. A systematic investigation of this kind has been conducted in Ref. [53]. In that work, the error of the predictions was 

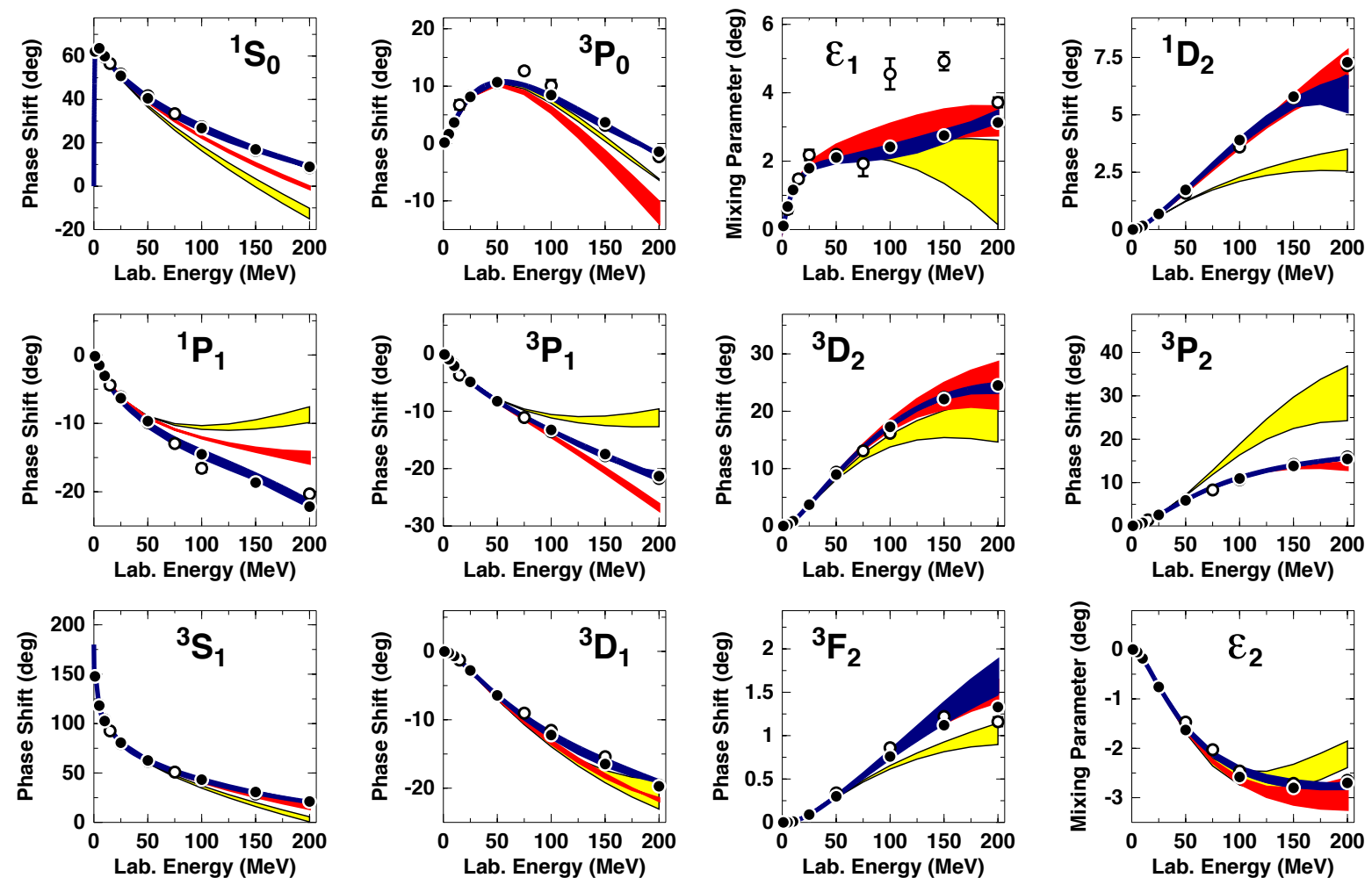

Figure 15. Phase shifts of neutron-proton scattering for the lower partial waves with $J \leq 2$. The yellow, red, and blue bands show the variations of the predictions with changing cutoffs between 450 and 600 at $\mathrm{NLO}, \mathrm{N}^{2} \mathrm{LO}$, and $\mathrm{N}^{3} \mathrm{LO}$, respectively. The predictions by $\mathrm{N}^{4} \mathrm{LO}$ potentials lie within the dark blue band and are, therefore, not explicitly shown. The solid dots and open circles are the results from the Nijmegen multi-energy $n p$ phase shift analysis [47] and the VPI/GW single-energy $n p$ analysis SM99 [48, respectively. (Figure reproduced from Ref. [66].)

quantified by calculating the $\chi^{2} /$ datum for the reproduction of the neutron-proton $(n p)$ elastic scattering data as a function of the cutoff parameter $\Lambda$ of the regulator function Eq. (96). Predictions by chiral $n p$ potentials at order NLO and NNLO were investigated applying Weinberg counting for the counter terms ( $N N$ contact terms). The results from this study for the energy range 35-125 MeV are shown in the upper frame of Fig. 14 and for $125-183 \mathrm{MeV}$ in the lower frame. It is seen that the reproduction of the $n p$ data at these energies is generally poor at NLO, while at NNLO the $\chi^{2} /$ datum assumes acceptable values (a clear demonstration of order-by-order improvement). Moreover, at NNLO one observes "plateaus" of constant low $\chi^{2}$ for cutoff parameters ranging from about 450 to $850 \mathrm{MeV}$. This may be perceived as cutoff independence (and, thus, successful renormalization) for the relevant range of cutoff parameters. 
Table 3. Columns three and four show the $\chi^{2} /$ datum for the reproduction of the $1999 n p$ database (defined in Ref. [44]) by families of $n p$ potentials at NLO and NNLO constructed by the Bochum group [38. The $\chi^{2} /$ datum is stated in terms of ranges which result from a variation of the cutoff parameters used in the regulator functions. The values of these cutoff parameters in units of $\mathrm{MeV}$ are given in parentheses. $T_{\text {lab }}$ denotes the kinetic energy of the incident neutron in the laboratory system.

\begin{tabular}{cccc}
\hline$T_{\text {lab }}(\mathrm{MeV})$ & \# of $n p$ data & \multicolumn{2}{c}{ - Bochum np potentials - } \\
Energy Bin & & NLO $(550 / 700-400 / 500)$ & NNLO $(600 / 700-450 / 500)$ \\
\hline $0-100$ & 1058 & $4-5$ & $1.4-1.9$ \\
$100-190$ & 501 & $77-121$ & $12-32$ \\
$190-290$ & 843 & $140-220$ & $25-69$ \\
\hline $0-290$ & 2402 & $67-105$ & $12-27$ \\
\hline
\end{tabular}

\subsection{NN potentials order by order}

As discussed, $N N$ potentials can be calculated at various orders, cf. Eqs. (33)-(38) and Eqs. (87)-(90), with better accuracy at higher orders. The convergence properties of the chiral expansion in the most central partial waves can be seen in Fig. 15. There, we display the $J \leq 2$ phase parameters for potentials constructed at NLO, NNLO, and $\mathrm{N}^{3} \mathrm{LO}$ with cutoffs ranging between 450 and $600 \mathrm{MeV}$. (The NLO and NNLO potentials are from Ref. [53] and the $\mathrm{N}^{3} \mathrm{LO}$ ones from Refs. [11, 62].) There is noticeable improvement in the agreement between the predictions and the empirical phase shifts as the order increases.

For a more direct comparison of theory and experiment, we can calculate observables, rather than phase shifts. The quality of the agreement with experimental data is typically expressed in terms of the $\chi^{2} /$ datum, with a value close to unity indicating a nearly perfect agreement.

In Table 3, we report the $\chi^{2}$ datum for the comparison beween the world $n p$ data below $290 \mathrm{MeV}$ and the predictions of $n p$ potentials at NLO and NNLO by the Bochum group [38]. The NLO potentials generate a very large $\chi^{2} /$ datum (between 67 and 105), while the NNLO potentials give values between 12 and 27, consistent with the findings of Ref. [53] shown in Fig. 14. It is promising to see that there is order-by-order improvement, but the $n p$ data at NLO and NNLO are not reproduced with sufficient quality. II

The most natural strategy is then to proceed to the next order, as suggested already in 2002 [54, 49]. The first $\mathrm{N}^{3} \mathrm{LO}$ potential followed shortly after [28].

At $\mathrm{N}^{3} \mathrm{LO}\left(Q^{4}\right), 24$ contact terms bring in a total of 24 parameters which impact partial waves with $L \leq 2$, while at NLO and NNLO there are only 9 contacts with $L \leq 1$ (cf. Section 6.1 and Table 44. These LECs are free constants employed to parametrize the short-range phenomenology. Table 4 shows how many terms with a certain power \| For an optimized NNLO potential see Ref. [63] and for local NLO and NNLO potentials see Ref. 64]. 
Table 4. Number of parameters needed for fitting the $n p$ data in the Nijmegen phaseshift analysis and by the high-precision CD-Bonn potential versus the total number of $N N$ contact terms of EFT based potentials to different orders.

\begin{tabular}{ccc|cccc}
\hline & $\begin{array}{c}\text { Nijmegen PWA93 } \\
\text { Ref [47] }\end{array}$ & $\begin{array}{c}\text { CD-Bonn pot. } \\
\text { Ref. [4] }\end{array}$ & \multicolumn{5}{c}{ EFT contact potentials [33] - } \\
& 3 & 4 & 1 & 2 & 4 & 6 \\
\hline${ }^{1} S_{0}$ & 3 & 4 & 1 & 2 & 4 & 6 \\
${ }^{3} S_{1}$ & 2 & 2 & 0 & 1 & 3 & 6 \\
\hline${ }^{3} S_{1^{-}}{ }^{3} D_{1}$ & 3 & 3 & 0 & 1 & 2 & 4 \\
\hline${ }^{1} P_{1}$ & 3 & 2 & 0 & 1 & 2 & 4 \\
${ }^{3} P_{0}$ & 2 & 2 & 0 & 1 & 2 & 4 \\
${ }^{3} P_{1}$ & 3 & 3 & 0 & 1 & 2 & 4 \\
${ }^{3} P_{2}$ & 2 & 1 & 0 & 0 & 1 & 3 \\
\hline${ }^{3} P_{2^{-}}{ }^{3} F_{2}$ & 2 & 3 & 0 & 0 & 1 & 2 \\
\hline${ }^{1} D_{2}$ & 2 & 1 & 0 & 0 & 1 & 2 \\
${ }^{3} D_{1}$ & 2 & 2 & 0 & 0 & 1 & 2 \\
${ }^{3} D_{2}$ & 1 & 2 & 0 & 0 & 1 & 2 \\
${ }^{3} D_{3}$ & 1 & 0 & 0 & 0 & 0 & 1 \\
${ }^{3} D_{3^{-}}{ }^{3} G_{3}$ & 1 & 1 & 0 & 0 & 0 & 1 \\
\hline${ }^{1} F_{3}$ & 1 & 2 & 0 & 0 & 0 & 1 \\
${ }^{3} F_{2}$ & 1 & 2 & 0 & 0 & 0 & 1 \\
${ }^{3} F_{3}$ & 2 & 1 & 0 & 0 & 0 & 1 \\
${ }^{3} F_{4}$ & 0 & 0 & 0 & 0 & 0 & 0 \\
\hline${ }^{3} F_{4^{-}}{ }^{3} H_{4}$ & 1 & 0 & 0 & 0 & 0 & 0 \\
\hline${ }^{1} G_{4}$ & 0 & 1 & 0 & 0 & 0 & 0 \\
${ }^{3} G_{3}$ & 0 & 1 & 0 & 0 & 0 & 0 \\
${ }^{3} G_{4}$ & 0 & 1 & 0 & 0 & 0 & 0 \\
${ }^{3} G_{5}$ & 35 & 38 & 2 & 9 & 24 & 50 \\
\hline Total & & & & & & 0 \\
\hline
\end{tabular}

of $Q$ participate in a given $N N$ state. One can see from the Table that contacts appear for the first time in $D$-waves at $\mathrm{N}^{3} \mathrm{LO}$. This is one important mechanism behind the considerable improvement in the reproduction of the $N N$ data at this order. Because the $D$-states are somewhat in between central and peripheral waves, contact terms, in addition to the one- and two-pion exchanges, are important to describe the $D$-phases correctly. Moreover, at $\mathrm{N}^{3} \mathrm{LO}$, every $P$-wave also benefits from an additional contact term, leading to further improvement, especially in ${ }^{3} P_{0}$ and ${ }^{3} P_{1}$ at incident laboratory energies greater than $100 \mathrm{MeV}$ (cf. Fig. 15).

Table 4 also displays the number of free parameters used in the Nijmegen partial wave analysis (PWA93) [47] and in the high-precision CD-Bonn potential [44]. For $S$ and 
Table 5. Columns three to five display the $\chi^{2} /$ datum for the reproduction of the 1999 $n p$ database (defined in Ref. [44]) by various $n p$ potentials. For the chiral potentials, the $\chi^{2} /$ datum is stated in terms of ranges which result from a variation of the cutoff parameters used in the regulator functions. The values of these cutoff parameters in units of $\mathrm{MeV}$ are given in parentheses. $T_{\text {lab }}$ denotes the kinetic energy of the incident nucleon in the laboratory system.

\begin{tabular}{ccccc}
\hline $\begin{array}{c}T_{\text {lab }}(\mathrm{MeV}) \\
\text { Energy Bin }\end{array}$ & \# of $\boldsymbol{n} \boldsymbol{p}$ data & $\begin{array}{c}\text { Idaho } \mathrm{N}^{3} \mathrm{LO} \\
(500-600)[28]\end{array}$ & $\begin{array}{c}\text { Bochum } \mathrm{N}^{3} \mathrm{LO} \\
(600 / 700-450 / 500)\end{array}$ & $\begin{array}{c}\text { Argonne } V_{18} \\
\text { Ref. [56] }\end{array}$ \\
\hline $0-100$ & 1058 & $1.0-1.1$ & $1.0-1.1$ & 0.95 \\
$100-190$ & 501 & $1.1-1.2$ & $1.3-1.8$ & 1.10 \\
$190-290$ & 843 & $1.2-1.4$ & $2.8-20.0$ & 1.11 \\
\hline $0-290$ & 2402 & $1.1-1.3$ & $1.7-7.9$ & 1.04 \\
\hline
\end{tabular}

$P$ waves, that number is approximately equal to the one required by EFT at $\mathrm{N}^{3} \mathrm{LO}\left(Q^{4}\right)$. Interestingly, we find in EFT a retroactive motivation for the phenomenology which became popular in the 1990's to construct high-precision potentials.

Thanks to the larger number of parameters, $\mathrm{N}^{3} \mathrm{LO}$ potentials can be constructed which are of about the same quality as the high-precision $N N$ potentials of the 1990's [44, 55, 56]. This fact is clearly revealed in the $\chi^{2} /$ datum for the fit of the $n p$ and $p p$ data below $290 \mathrm{MeV}$ shown in Table 5 and 6 , respectively. Table 5, which is pretty self-explanatory, displays the $\chi^{2} /$ datum for various chiral potentials as well as the Argonne potential, compared with the world $n p$ data below $290 \mathrm{MeV}$.

As we turn now to $p p$, note first that the $\chi^{2}$ for $p p$ data are typically larger than for $n p$ because of the higher precision of $p p$ data (Table 6). Thus, the Argonne $V_{18}$ produces a $\chi^{2} /$ datum $=1.4$ for the world $p p$ data below $290 \mathrm{MeV}$ and the best Idaho $\mathrm{N}^{3} \mathrm{LO} p p$ potential obtains 1.5. The fit by the best Bochum $\mathrm{N}^{3} \mathrm{LO} p p$ potential results in a $\chi^{2} /$ datum $=2.9$ and the worst produces 22.3 . In view of these poor $\chi^{2}$, the Bochum group has recently launched an attempt towards improving their chiral potentials [58, 59]. However, as in their previous work [57], they have fitted their new potentials only to $N N$ phase shifts and not to the $N N$ data. The $\chi^{2}$ for the reproduction of the $N N$ data by the new Bochum potentials are not available and,

Table 6. Same as Table 5 but for $\boldsymbol{p p}$.

\begin{tabular}{ccccc}
\hline $\begin{array}{c}T_{\text {lab }}(\mathrm{MeV}) \\
\text { Energy Bin }\end{array}$ & \# of $\boldsymbol{p} \boldsymbol{p}$ data & $\begin{array}{c}\text { Idaho } \mathrm{N}^{3} \mathrm{LO} \\
(500-600)[28\end{array}$ & $\begin{array}{c}\text { Bochum } \mathrm{N}^{3} \mathrm{LO} \\
(600 / 700-450 / 500)\end{array}$ & $\begin{array}{c}\text { Argonne } V_{18} \\
\text { Ref. [56] }\end{array}$ \\
\hline $0-100$ & 795 & $1.0-1.7$ & $1.0-3.8$ & 1.0 \\
$100-190$ & 411 & $1.5-1.9$ & $3.5-11.6$ & 1.3 \\
$190-290$ & 851 & $1.9-2.7$ & $4.3-44.4$ & 1.8 \\
\hline $0-290$ & 2057 & $1.5-2.1$ & $2.9-22.3$ & 1.4 \\
\hline
\end{tabular}


thus, no reliable statement about the quality of the new potentials can be made. In the 1990's, the Nijmegen group has pointed out repeatedly that for high quality potentials

it is insufficient to fit phase shifts only. A seemingly "good" fit of phase shifts can be misleading and can result in a poor $\chi^{2}$ for the reproduction of the data.

Concerning alternative $\mathrm{N}^{3} \mathrm{LO}$ potentials, we note that a minimally non-local $N N$ potential of this kind has been constructed in Ref. [65] which produces a $\chi^{2} /$ datum of about 1.3 for the $p p$ plus $n p$ data.

Now turning to $\mathrm{N}^{4} \mathrm{LO}$ : Based upon the derivation of the $2 \mathrm{PE}$ and $3 \mathrm{PE}$ contributions to the $N N$ interaction at $\mathrm{N}^{4} \mathrm{LO}$ by Entem et al. [29] presented in Sec. 4.5 and applied in peripheral scattering in Sec. 5, $N N$ potential at $\mathrm{N}^{4} \mathrm{LO}$ have recently been developed [29, 59]. Note that the lower partial waves, which are crucial for a quantitative reproduction of the $N N$ data, are ruled by the contact terms. The number of contacts at $\mathrm{N}^{4} \mathrm{LO}\left(Q^{5}\right)$ is the same as at $\mathrm{N}^{3} \mathrm{LO}\left(Q^{4}\right)$. Thus, the $\mathrm{N}^{4} \mathrm{LO}$ potentials are not very different from the $\mathrm{N}^{3} \mathrm{LO}$ ones. Note also that the high quality of some of the $\mathrm{N}^{3} \mathrm{LO}$ potentials [11, 28, 65] leaves little room for improvements.

A further increase in accuracy (if needed) could be achieved at $\mathrm{N}^{5} \mathrm{LO}\left(Q^{6}\right)$, where the number of contact terms advances to 50 (Table 4) [33]. As discussed in Sec. 4.6, the dominant $2 \mathrm{PE}$ and $3 \mathrm{PE}$ contributions at $\mathrm{N}^{5} \mathrm{LO}$ have been derived [32]. Thus, all the mathematical material for the construction of $\mathrm{N}^{5} \mathrm{LO}$ potentials is available. However, it is debatable if there is a need for them.

\section{Nuclear many-body forces}

Two-nucleon forces derived from chiral EFT as described above have been applied, often successfully, in the many-body system. On the other hand, over the past several years we have learnt that, for some few-nucleon reactions and nuclear structure issues, $3 N F$ s cannot be neglected. The most well-known cases are the so-called $A_{y}$ puzzle of $N-d$ scattering [60], the ground state of ${ }^{10} \mathrm{~B}$ [61], and the saturation of nuclear matter [62, 66]. As we observed previously, the EFT approach generates consistent two- and manynucleon forces in a natural way (cf. the overview given in Fig. 1). We now shift our focus to chiral three- and four-nucleon forces.

\subsection{Three-nucleon forces}

Weinberg [10] was the first to discuss nuclear three-body forces. Not long after that, the first $3 \mathrm{NF}$ at NNLO was derived by van Kolck [24].

For a $3 \mathrm{NF}$, we have $A=3$ and $C=1$ and, thus, Eq. (25) implies

$$
\nu=2+2 L+\sum_{i} \Delta_{i}
$$

We will use this equation to analyze $3 \mathrm{NF}$ contributions order by order. 


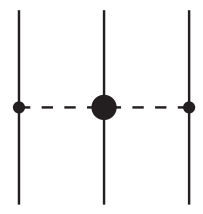

(a)

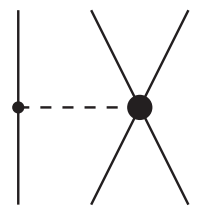

(b)

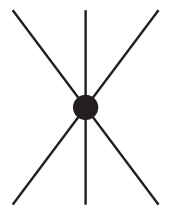

(c)

Figure 16. The three-nucleon force at NNLO with (a) 2PE, (b) 1PE, and (c) contact diagrams. Notation as in Fig. 1. (Figure reproduced from Ref. [66.)

7.1.1. Next-to-leading order The lowest possible power is obviously $\nu=2$ (NLO), which is obtained for no loops $(L=0)$ and only leading vertices $\left(\sum_{i} \Delta_{i}=0\right)$. As discussed by Weinberg [10] and van Kolck [24], the contributions from these diagrams vanish at NLO. So, the bottom line is that there is no genuine $3 \mathrm{NF}$ contribution at NLO. The first non-vanishing 3NF appears at NNLO.

7.1.2. Next-to-next-to-leading order The power $\nu=3$ (NNLO) is obtained when there are no loops $(L=0)$ and $\sum_{i} \Delta_{i}=1$, i.e., $\Delta_{i}=1$ for one vertex while $\Delta_{i}=0$ for all other vertices. There are three topologies which fulfill this condition, known as the $2 \mathrm{PE}, 1 \mathrm{PE}$, and contact graphs [24, 25] (Fig. 16).

The $2 \mathrm{PE} 3 \mathrm{~N}$-potential is derived to be

$$
V_{2 \mathrm{PE}}^{3 \mathrm{NF}}=\left(\frac{g_{A}}{2 f_{\pi}}\right)^{2} \frac{1}{2} \sum_{i \neq j \neq k} \frac{\left(\vec{\sigma}_{i} \cdot \vec{q}_{i}\right)\left(\vec{\sigma}_{j} \cdot \vec{q}_{j}\right)}{\left(q_{i}^{2}+m_{\pi}^{2}\right)\left(q_{j}^{2}+m_{\pi}^{2}\right)} F_{i j k}^{a b} \tau_{i}^{a} \tau_{j}^{b}
$$

with $\vec{q}_{i} \equiv \vec{p}_{i}^{\prime}-\vec{p}_{i}$, where $\vec{p}_{i}$ and $\vec{p}_{i}^{\prime}$ are the initial and final momenta of nucleon $i$, respectively, and

$$
F_{i j k}^{a b}=\delta^{a b}\left[-\frac{4 c_{1} m_{\pi}^{2}}{f_{\pi}^{2}}+\frac{2 c_{3}}{f_{\pi}^{2}} \vec{q}_{i} \cdot \vec{q}_{j}\right]+\frac{c_{4}}{f_{\pi}^{2}} \sum_{c} \epsilon^{a b c} \tau_{k}^{c} \vec{\sigma}_{k} \cdot\left[\vec{q}_{i} \times \vec{q}_{j}\right] .
$$

It is interesting to observe that there are clear analogies between this force and earlier 2PE 3NFs already proposed decades ago, particularly the Fujita-Miyazawa [67] and the Tucson-Melbourne (TM) 68] forces.

The $2 \mathrm{PE} 3 \mathrm{NF}$ does not introduce additional fitting constants, since the LECs $c_{1}$, $c_{3}$, and $c_{4}$ are already present in the $2 \mathrm{PE} 2 \mathrm{NF}$. These LECs are constrained by $N N$ and $\pi N$ data.

The other two 3NF contributions shown in Fig. 16 are easily derived by taking the last two terms of the $\Delta=1$ Langrangian, Eq. (18), into account. The 1PE contribution is

$$
V_{1 \mathrm{PE}}^{3 \mathrm{NF}}=-D \frac{g_{A}}{8 f_{\pi}^{2}} \sum_{i \neq j \neq k} \frac{\vec{\sigma}_{j} \cdot \vec{q}_{j}}{q_{j}^{2}+m_{\pi}^{2}}\left(\boldsymbol{\tau}_{i} \cdot \boldsymbol{\tau}_{j}\right)\left(\vec{\sigma}_{i} \cdot \vec{q}_{j}\right)
$$

and the $3 \mathrm{~N}$ contact potential is given by

$$
V_{\mathrm{ct}}^{3 \mathrm{NF}}=E \frac{1}{2} \sum_{i \neq j \neq k} \boldsymbol{\tau}_{i} \cdot \boldsymbol{\tau}_{j}
$$




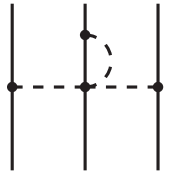

(a)

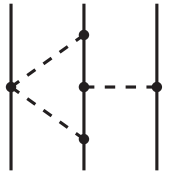

(b)

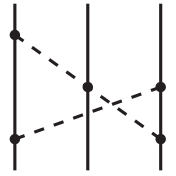

(c)

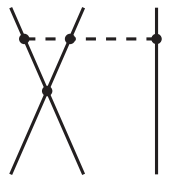

(d)

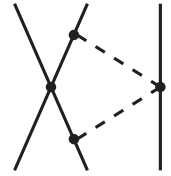

(e)

Figure 17. Leading one-loop $3 \mathrm{NF}$ diagrams at $\mathrm{N}^{3} \mathrm{LO}$. We show one representative example for each of five topologies, which are: (a) 2PE, (b) 1PE-2PE, (c) ring, (d) contact-1PE, (e) contact-2PE. Notation as in Fig. 1.

These $3 \mathrm{NF}$ potentials introduce two additional constants, $D$ and $E$, which can be constrained in more than one way. One may use the triton binding energy and the nd doublet scattering length ${ }^{2} a_{n d}$ as done in Ref. [25. Alternative choices include the binding energies of ${ }^{3} \mathrm{H}$ and ${ }^{4} \mathrm{He}$ [69] or an optimal global fit of the properties of light nuclei [70]. Another method makes use of the triton binding energy and the Gamow-Teller matrix element of tritium $\beta$-decay [71. When the values of $D$ and $E$ are determined, the results for other observables involving three or more nucleons are true theoretical predictions.

Applications of the leading 3NF include few-nucleon reactions, spectra of lightand medium-mass nuclei [72, 73], and nuclear and neutron matter [62, 66], often with satisfactory results. Some problems, though, remain unresolved, such as the well-known ' $A_{y}$ puzzle' in nucleon-deuteron scattering [60, 25]. Predictions which employ only 2 NFs underestimate the analyzing power in $p^{-}{ }^{3} \mathrm{He}$ scattering to a larger degree than in $p-d$. Although the $p-{ }^{3} \mathrm{He} A_{y}$ improves considerably (more than in the $p$ - $d$ case) when the leading $3 \mathrm{NF}$ is included [74, the disagreement with the data is not fully removed. Also, predictions for light nuclei are not quite satisfactory [70].

In summary, the leading $3 \mathrm{NF}$ of $\mathrm{ChPT}$ is an outstanding contribution. It gives validation to, and provides a better framework for, 3NFs which were proposed already 5 decades ago; it alleviates existing problems in few-nucleon reactions and the spectra of light nuclei. Nevertheless, we still face several challenges. With regard to the $2 \mathrm{NF}$, we have discussed earlier that it is necessary to go to order 4 for high-quality predictions. Thus, the $3 \mathrm{NF}$ at $\mathrm{N}^{3} \mathrm{LO}$ must be considered simply as a matter of consistency with the $2 \mathrm{NF}$ sector. At the same time, one hopes that its inclusion may result in further improvements with the aforementioned unresolved problems.

7.1.3. Next-to-next-to-next-to-leading order At $\mathrm{N}^{3} \mathrm{LO}$, there are loop and tree diagrams. For the loops (Fig. 17), we have $L=1$ and, therefore, all $\Delta_{i}$ have to be zero to ensure $\nu=4$. Thus, these one-loop 3NF diagrams can include only leading order vertices, the parameters of which are fixed from $\pi N$ and $N N$ analysis. One subgroup of these diagrams (the 2PE graphs, cf. Fig. 17) has been calculated by Ishikawa and Robilotta [75], and the other topologies have been evaluated by the Bochum-Bonn group [76]. The $\mathrm{N}^{3} \mathrm{LO} 2 \mathrm{PE} 3 \mathrm{NF}$ has been applied in the calculation of nucleon-deuteron observables in Ref. [75] causing little impact. Very recently, the long-range part of the 


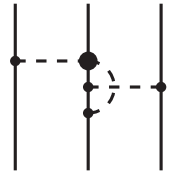

(a)

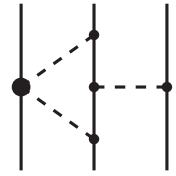

(b)

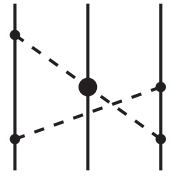

(c)

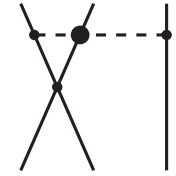

(d)

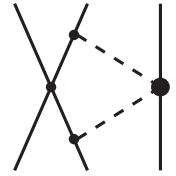

(e)

Figure 18. Sub-leading one-loop $3 \mathrm{NF}$ diagrams which appear at $\mathrm{N}^{4} \mathrm{LO}$ with topologies similar to Fig. 17. Notation as in Fig. 1.

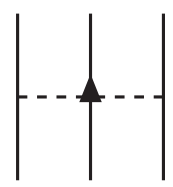

(a)

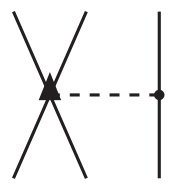

(b)

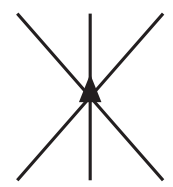

(c)

Figure 19. $3 \mathrm{NF}$ tree graphs at $\mathrm{N}^{4} \mathrm{LO}(\nu=5)$ denoted by: (a) 2PE, (b) 1PE-contact, and (c) contact. Notation as in Fig. 1 .

chiral $\mathrm{N}^{3} \mathrm{LO} 3 \mathrm{NF}$ has been tested in the triton [77] and in three-nucleon scattering [78] yielding only moderate effects. The long- and short-range parts of this force have been used in neutron matter calculations (together with the $\mathrm{N}^{3} \mathrm{LO} 4 \mathrm{NF}$ ) producing relatively large contributions from the 3NF [79]. Thus, the ultimate assessment of the $\mathrm{N}^{3} \mathrm{LO} 3 \mathrm{NF}$ is still outstanding and will require more few- and many-body applications.

7.1.4. The $3 N F$ at $N^{4} L O$ In the meantime, one may go ahead and look at the next order of $3 \mathrm{NFs}$, which is $\mathrm{N}^{4} \mathrm{LO}$ or $\nu=5$. The loop contributions that occur at this order are obtained by replacing in the $\mathrm{N}^{3} \mathrm{LO}$ loops one vertex by a $\Delta_{i}=1$ vertex (with LEC $c_{i}$ ), Fig. 18, which is why these loops may be more sizable than the $\mathrm{N}^{3} \mathrm{LO}$ loops. The 2PE, 1PE-2PE, and ring topologies have been evaluated [22, 30] so far. In addition, we have three 'tree' topologies (Fig. 19), which include a new set of $3 \mathrm{~N}$ contact interactions that has recently been derived by the Pisa group [31]. Contact terms are typically simple (as compared to loop diagrams) and their coefficients are essentially free. Therefore, it would be an attractive project to test some terms (in particular, the spin-orbit terms) of the $\mathrm{N}^{4} \mathrm{LO}$ contact $3 \mathrm{NF}$ [31] in calculations of few-body reactions (specifically, the $p$ - $d$ and $\left.p-{ }^{3} \mathrm{He} A_{y}\right)$ and spectra of light nuclei.

\subsection{Four-nucleon forces}

For connected $(C=1) A=4$ diagrams, Eq. 25) yields

$$
\nu=4+2 L+\sum_{i} \Delta_{i}
$$

We then see that the first (connected) non-vanishing $4 \mathrm{NF}$ is generated at $\nu=4\left(\mathrm{~N}^{3} \mathrm{LO}\right)$, with all vertices of leading type, Fig. 20. This $4 \mathrm{NF}$ contribution has no loops and 

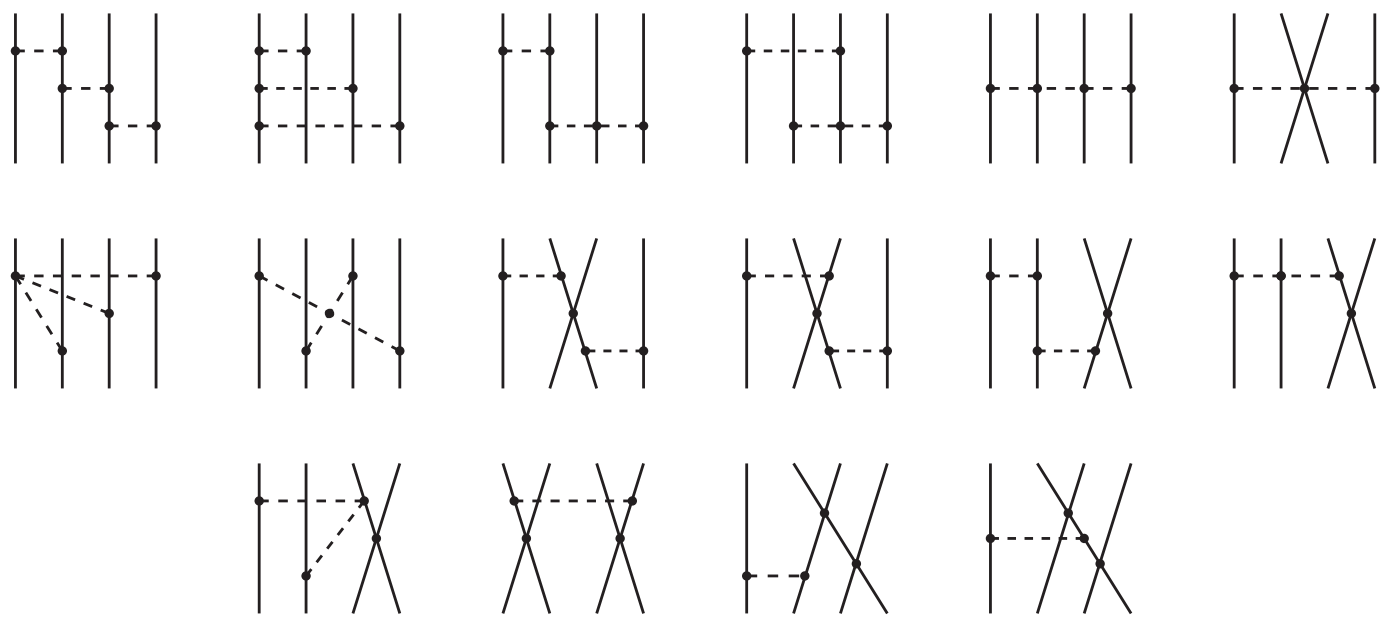

Figure 20. Leading four-nucleon force at $\mathrm{N}^{3} \mathrm{LO}$. (Figure reproduced from Ref. [11].)

introduces no novel parameters [80]. (See Ref. [11] for a more detailed discussion on these diagrams.)

For a reasonably convergent series, terms of order $\left(Q / \Lambda_{\chi}\right)^{4}$ must be small, and therefore chiral $4 \mathrm{NF}$ are predicted to be very weak. This expectation was confirmed in a recent calculation of the ${ }^{4} \mathrm{He}$ binding energy including the leading $4 \mathrm{NF}$ (Fig. 20). Its

effect was found to be a few $100 \mathrm{keV}$ [81, to be compared with the actual size of the binding energy, 28.3 MeV. Although obtained with the help of several approximations, this preliminary predictions supports the notion that $4 \mathrm{NF}$ may indeed be negligeable.

The effects of the leading chiral $4 \mathrm{NF}$ in symmetric nuclear matter and pure neutron matter have been worked out by Kaiser et al. [82, 83].

\section{Applications in the nuclear many-body problem}

In this Section, we will address some recent applications of the few-nucleon forces which were derived and discussed in previous sections. In particular, we will concentrate on applications where the analysis is conducted in the spirit of exploring order-by-order convergence of the predictions.

It should be clear from the historical perspectives presented at the opening of this article that our present knowledge of nuclear forces in free space and in the fewnucleon system is the result of decades of struggle. The nature of the nuclear force in a dense medium is an even more complex problem, as it involves aspects of the forces that cannot be constrained through free-space $N N$ scattering or the properties of the (relatively "simple") few-nucleon system.

Although predictions for finite nuclei are the ultimate test for many-body predictions, infinite nuclear matter is an alternative and convenient testing ground for many-body theories. By "nuclear matter" we mean an infinite system of nucleons acted on by their mutual strong forces and no electromagnetic interactions. Nuclear matter 
is characterized by its energy per particle as a function of density and other quantities as appropriate (e.g. temperature, isospin asymmetry, spin asymmetry). Such relation is known as the nuclear matter equation of state (EoS). The translational invariance of the system facilitates theoretical calculations. At the same time, adopting what is known as "local density approximation", one may use the EoS directly in calculations of finite systems, as we will discuss below.

When proton and neutron densities are different (that is, in the presence of isospin asymmetry), the energy per particle becomes a function of both the total density and the relative concentrations of neutrons and protons. The EoS of isospin-asymmetric matter naturally introduces the symmetry energy, similarly to the appearance of the symmetry term in the well-known Bethe-Weizäcker formula. As will be discussed later in more details, the symmetry energy is typically approximated as the difference between the energy per particle of symmetric nuclear matter and pure neutron matter as a function of density. Of particular contemporary interest is the EoS of highly neutron-rich matter, all the way to nearly pure neutron matter. This quantity is important for understanding wide ranging questions in modern nuclear physics, from the properties of rare isotopes to those of neutron stars. On the one hand, the density dependence of the symmetry energy is known to correlate strongly with the neutron skin thickness of a heavy nucleus. On the other hand, the pressure in neutron-rich matter is the main input for the structure equations of compact stars. Therefore, microscopic predictions together with empirical constraints from observables that are sensitive to the equation of state are an ideal combination to learn about the in-medium behavior of nuclear forces, particularly in isospin-asymmetric medium. We also recall that the EoS is an important part of the input of transport models describing heavy-ion collisions and thus can be constrained through analyses of carefully selected observables in ion-ion scattering. Concerning non-terrestrial observations, partnership between nuclear physics and astrophysics is increasingly important as better constraints on the high-density part of the equation of state become available through more accurate measurements of neutron star masses. In summary, studies of nucleonic matter are especially timely and important, as they support rich on-going and future experimental effort, both in terrestrial laboratories and the cosmos.

\subsection{Order-by-order predictions of the energy per nucleon in nuclear and neutron matter}

The problem shared by all non-EFT based approaches is that it is essentially impossible to estimate reliably the uncertainty associated with a particular prediction. On the other hand, EFT provides a well-defined framework to calculate observables where the truncation error decreases systematically as higher orders are included. Earlier in this article, we have seen that such task can be accomplished quite successfully at the level of $N N$ phase shifts.

In this section, we will review and discuss recent calculations of the energy per 
particle in infinite matter at different orders of chiral EFT [66]. The discussion will also emphasize the importance of error quantification and how it should be addressed in chiral EFT.

Estimates of theoretical uncertainties [84] for calculations of the equation of state have mostly focused on varying the low-energy constants and resolution scale at which nuclear dynamics are probed [85, 86, 62, 87, 88, 89]. In a recent work [66] we layed the foundation for order-by-order calculations of nuclear many-body systems by presenting consistent NLO and $\mathrm{N}^{2} \mathrm{LO}$ chiral nuclear forces whose relevant short-range three-body forces are fit to $\mathrm{A}=3$ binding energies and the lifetime of the triton. We then assessed the accuracy with which infinite nuclear matter properties and the isospin asymmetry energy can be predicted from order-by-order calculations in chiral effective field theory.

Uncertainty originates from:

- The choice of the many-body method (a source of error not inherent to EFT).

- Error in the determination of the low-energy constants (LECs). Short-range LECs $(N N)$ and long-range LECs $(\pi N)$ must be considered separately.

- Regulator dependence.

- Truncation error.

In the following, we will address those items briefly but systematically.

A variety of many-body methods are available and have been used extensively in nuclear matter predictions. They include: the coupled-cluster method, manybody perturbation theory, variational Monte Carlo or Greens function Monte Carlo methods. In computing the EoS, we employ the nonperturbative particle-particle ladder approximation. In the traditional hole-line expansion, it represents the leadingorder contribution. To quantify the uncertainty carried by this choice, it is insightful to compare with Refs. [90, 91]. In Ref. [90], the authors report on coupled-cluster calculations in symmetric nuclear matter including particle-particle (pp) and hole-hole (hh) diagrams (as well as an exact treatment of the Pauli operator). The overall effect, as seen from comparing the first and last entries in Table II of Ref. [90], is very small around saturation density, consistent with Table II in Ref. [62], and grows to $1.5 \mathrm{MeV}$ at the highest Fermi momentum included in the study. Note that these calculations adopt the $\mathrm{N}^{3} \mathrm{LO}$ potential [28] (with $\Lambda=500 \mathrm{MeV}$ ) and no three-nucleon forces. On the other hand, in Ref. 91] coupled-cluster calculations in nucleonic matter were performed at $\mathrm{N}^{2} \mathrm{LO}$ with two- and three-body forces and with the inclusion of selected triples clusters, namely correlations beyond pp and hh ladders. The effect of these contributions is found to be negligible in neutron matter and about $1 \mathrm{MeV}$ per nucleon in symmetric matter in the density range under consideration [91. In the light of the above considerations, we conclude that a realistic estimate of the impact of using a nonperturbative approach beyond pp correlations is about $1 \mathrm{MeV}$ in nuclear matter around saturation density and much smaller in neutron matter. As we show below, such uncertainties are significantly smaller than those associated with variations in the cutoff scale. 
In order to quantify the error associated with possible variations of the (shortrange) $N N$ LECs, we refer to recent findings from the Granada group [92]. They applied 205 samples of smooth local potentials, all with $\chi^{2} /$ datum of approximately 1, and found a variation of $15 \mathrm{keV}$ in the triton binding energy. From our part, we performed Brueckner-Hartree-Fock calculations in nuclear matter using local highprecision potentials from the Nijmegen group [55] and observed an uncertainty of 0.6 $\mathrm{MeV}$ in the energy per particle at normal density. In summary, we conclude that the uncertainty arising from the error in the $N N$ data has negligible impact on the manybody system. Concerning the (long-range) $\pi N$ LECs, they are likely to impact mostly peripheral partial waves (namely, those high partial waves where no contact terms are present). At $\mathrm{NLO}$ and $\mathrm{N}^{2} \mathrm{LO}$, that means $D$-waves and higher, whereas at $\mathrm{N}^{3} \mathrm{LO}$ no contacts exist in $F$-waves and higher. Therefore, we expect variations of the $\pi N$ LECs (within the range allowed by $\pi N$ scattering data) to have only minor impact in nuclear matter, since its sensitivity is limited to peripheral partial waves. Nevertheless, we stress that a systematic investigation with consideration of $\pi N$ LECs uncertainty consistently in the $2 \mathrm{NF}$ and the $3 \mathrm{NF}$, has not yet been done and is part of our future plans.

Keeping in mind the uncertainty considerations made above, we now move to nuclear and neutron matter predictions. Our results for the energy per particle as a function of the nuclear density are shown in Fig. 21 for symmetric nuclear matter. We note that the particle-particle ladder approximation employed in the present work is in good agreement with the perturbative results available at $\mathrm{N}^{3} \mathrm{LO}$ from Ref. [62] including up to third-order pp diagrams. In Fig. 21, the shaded bands in yellow and red represent the spread of our complete calculations conducted at NLO and $\mathrm{N}^{2} \mathrm{LO}$, respectively. The blue band is the result of a calculation that employs $\mathrm{N}^{3} \mathrm{LO} N N$ potentials together with $\mathrm{N}^{2} \mathrm{LO} 3 \mathrm{NFs}$. In all cases shown, the cutoff is varied over the range $450-600 \mathrm{MeV}$. As noted before, the $\mathrm{N}^{3} \mathrm{LO} 3 \mathrm{NFs}$ and $4 \mathrm{NFs}$ are at present omitted, and the resulting convergence pattern gives an estimate on the theoretical uncertainty of the calculation (and not necessarily of the chiral effective field theory expansion per se). We note that at NLO the potentials constructed at lower cutoff scales do not exhibit saturation until very high densities. On the other hand, for the $600 \mathrm{MeV}$ cutoff potential the ${ }^{1} S_{0}$ partial wave (together with the ${ }^{3} S_{1}$ partial wave) is sufficiently repulsive to enable saturation at a relatively smaller density. We observe that the convergence pattern for the low-cutoff $(\Lambda=450-500 \mathrm{MeV})$ potentials is significantly better than for the 600 $\mathrm{MeV}$ potential. Overall there is a large spread from cutoff variations both at NLO and $\mathrm{N}^{2} \mathrm{LO}$ beyond nuclear matter saturation density. Moreover, the bands at these two orders do not overlap, suggesting that their width is not a suitable representation of the uncertainty. Although the (not yet complete) $\mathrm{N}^{3} \mathrm{LO}$ calculation reveals a strong reduction of the cutoff dependence, it is important to notice that an uncertainty of about $8 \mathrm{MeV}$ remains at saturation density. While we do not expect much of a change in nuclear matter predictions from 4NFs [80, 81, 86, it is quite possible that the inclusion of $\mathrm{N}^{3} \mathrm{LO} 3 \mathrm{NFs}$ might reduce either the cutoff dependence or improve the convergence pattern. This will be an interesting subject for future investigations. 

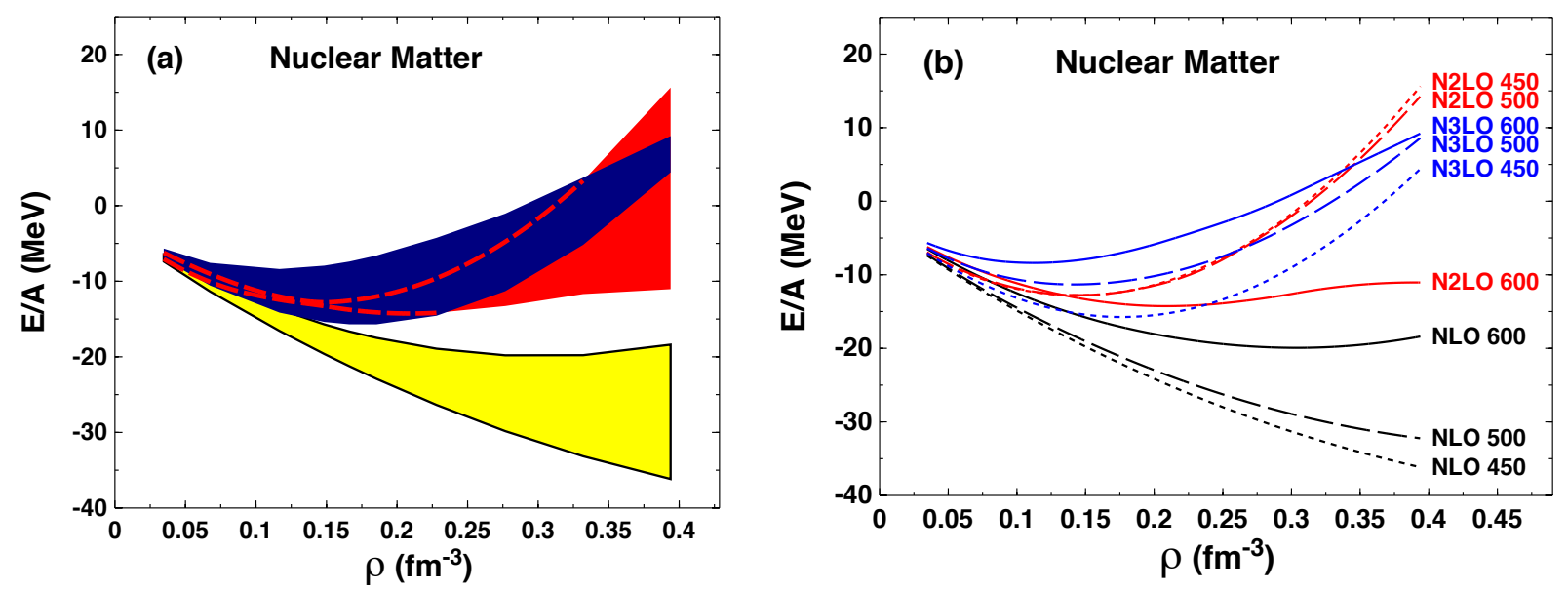

Figure 21. Energy/nucleon (E/A) in symmetric nuclear matter as a function of density, $\rho$. Left frame: The yellow and red bands represent the uncertainties in the predictions due to cutoff variations as obtained in complete calculations at NLO and $\mathrm{N}^{2} \mathrm{LO}$, respectively. The blue band is the result of a calculation employing $\mathrm{N}^{3} \mathrm{LO} N N$ potentials together with $\mathrm{N}^{2} \mathrm{LO} 3 \mathrm{NFs}$. The dashed lines show the upper or lower limits of hidden bands. Right frame: predictions at the specified order and cutoff value. (Figure reproduced from Ref. [66].)

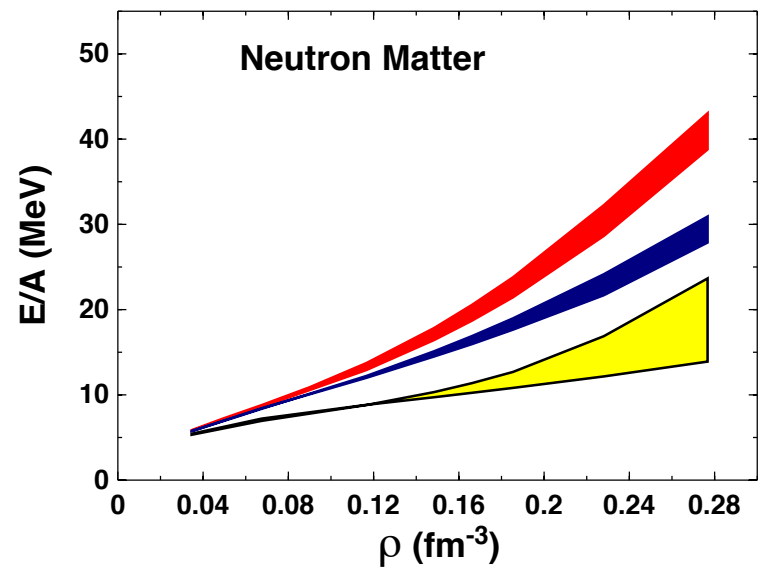

Figure 22. As in Fig. 21 for pure neutron matter. (Figure reproduced from Ref. [66].)

The results for neutron matter are presented in Fig. 22, where the bands have the same meaning as in Fig. 21. Note that the range of densities under consideration is smaller for neutron matter in order to keep the Fermi momentum below the cutoff in all cases. We see a large spread at NLO for the largest densities considered, whereas the band has only moderate size at the next order and remains small for our $\mathrm{N}^{3} \mathrm{LO}$ calculation. Similar to what was observed in symmetric nuclear matter, the bands at $\mathrm{NLO}$ and $\mathrm{N}^{2} \mathrm{LO}$ do not overlap in neutron matter. In addition, the $\mathrm{N}^{3} \mathrm{LO}$ band does not generally overlap with the $\mathrm{N}^{2} \mathrm{LO}$ band. Therefore, the variation obtained by changing 


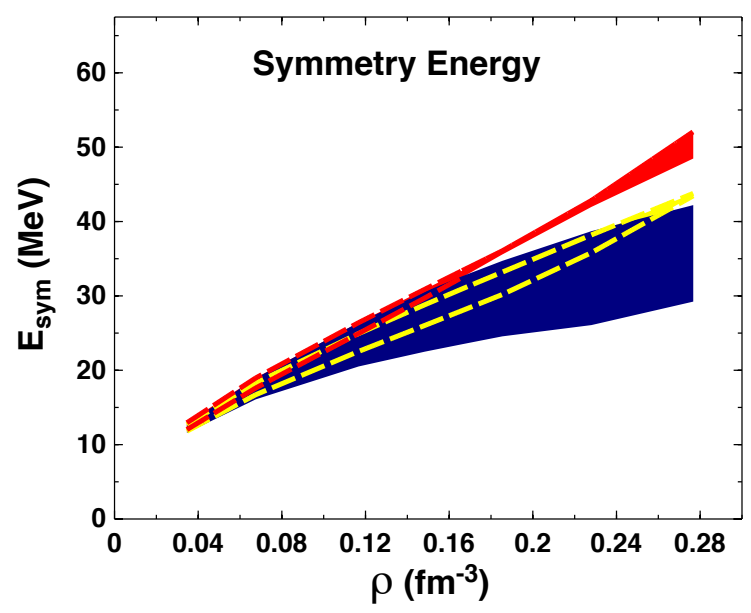

Figure 23. The symmetry energy, $E_{\mathrm{sym}}$, as a function of density, $\rho$. Meaning of bands and dashed lines as in Fig. 21. (Figure reproduced from Ref. [66.)

the cutoff does not seem to provide a reliable representation of the uncertainty at the given order. A better way to estimate such uncertainty is to consider the difference between the predictions at two consecutive orders.

In Fig. 23 we present the results for the symmetry energy, which is defined as the strength of the quadratic term in an expansion of the energy per particle in asymmetric matter with respect to the asymmetry parameter $\alpha$ :

$$
\bar{E}(\rho, \alpha) \approx \bar{E}(\rho, \alpha=0)+E_{\mathrm{sym}} \alpha^{2}+\mathcal{O}\left(\alpha^{4}\right),
$$

where $\bar{E}=E / A$ is the energy per particle and $\alpha=\left(\rho_{n}-\rho_{p}\right) /\left(\rho_{n}+\rho_{p}\right)$. The nearly linear behavior of $\bar{E}(\rho, \alpha)$ with $\alpha^{2}$ has been confirmed by many microscopic calculations (see for instance Refs. [93, 94], but see also Ref. [95]). It is a common approximation to neglect powers beyond $\alpha^{2}$ in the expansion above and thus defining the symmetry energy as the difference between the energy per particle in neutron matter and symmetric nuclear matter.

As mentioned at the beginning of this section, systematic efforts are ongoing to set better empirical constraints on the symmetry energy, through both laboratory and astrophysical measurements. It is therefore important to have an understanding of the theoretical uncertainty affecting calculations of this quantity. The spread due to the change of the cutoff values in our NLO, $\mathrm{N}^{2} \mathrm{LO}$, and $\mathrm{N}^{3} \mathrm{LO}$ calculations is represented by the three bands as before. As observed previously for symmetric matter, the spread due to cutoff variations remains large at $\mathrm{N}^{2} \mathrm{LO}$, with some minimal overlap with the NLO band. The $\mathrm{N}^{3} \mathrm{LO}$ band reflects the large cutoff sensitivity previously observed in symmetric matter. Again, we conclude that the spread generated by changing the cutoff does not in general provide a reliable estimate of the theoretical uncertainty. 


\subsection{Spin-polarized neutron matter}

Polarized neutron matter (NM) is an interesting system for various reasons. Among them is the impact that spin instabilities in the interior of stellar matter would have on neutrino interactions and thus the star cooling mechanism.

Spin polarization is also of interest in symmetric or nearly symmetric nuclear matter (SNM). For instance, for the purpose of scattering from polarized nuclei, one may define a spin dependent optical potential which, for the spin degree of freedom, plays the same role as the Lane potential [97] for the isospin degree of freedom. Such spin symmetry potential can be obtained from the difference between the single-particle potentials for spin-up and spin-down nucleons in polarized SNM.

To address the most general case, one must include both spin and isospin polarizations. From the astrophysics point of view, stellar matter contains a small, but not insignificant proton fraction. With regard to experiments in terrestrial laboratories, the spin dependence of the nuclear interaction in nuclear matter can be explored through collective exitations, such as giant resonances. Most typically, a nucleus with non-zero spin is also isospin asymmetric, making it necessary to include both spin and isospin polarizations. For those reasons, in previous work [96, 98, 99] we explored matter with different densities of neutrons and protons where each type of nucleon can have arbitrary degree of spin polarization. We obtained predictions employing the Dirac-BruecknerHartree-Fock approach to nuclear matter and a relativistic one-boson-exchange $N N$ potential and did not see any indications of a phase transition to a spin-polarized state. We note that all models which start from the bare $N N$ force and apply it in the medium (see, for instance, Ref. [100]) end up with similar conclusions. In contrast, approaches based on parametrizations of Skyrme forces, or other phenomenological forces, report different findings. For instance, with the SLy 4 and SLy5 forces and the Fermi liquid formalism a phase transition to the antiferromagnetic state is predicted in asymmetric matter at a critical density equal to about 2-3 times normal density [101]. Qualitative disagreement is also encountered with other approaches such as relativistic Hartree-Fock models based on effective meson-nucleon Lagrangians. For instance, in Ref. [102] it was reported that the onset of a ferromagnetic transition in neutron matter, and its critical density, are crucially determined by the inclusion of isovector mesons and the nature of their couplings.

The brief review given above summarizes the findings of many useful and valid calculations. However, the problem common to all of them is that it is essentially impossible to estimate, in a statistically meaningful way, the uncertainties associated with a particular prediction, or to quantify the error related to the approximations applied in a particular model. Therefore, in this section, we apply the same philosophy as in Sec. 8.1 to study the equation of state of polarized neutron matter at different orders of ChPT.

Based on the literature mentioned above, a phase transition to a polarized phase

(at least up to normal densities) seems unlikely, although the validity of such conclusion 
must be assessed in the context of EFT errors. Furthermore, polarized neutron matter is a very interesting system for several reasons. Because of the large neutron-neutron scattering length, NM displays behaviors similar to those of a unitary Fermi gas. In fact, up to nearly normal density, (unpolarized) neutron matter is found to display the behavior of an $S$-wave superfluid [103, 104]. The possibility of simulating low-density NM with ultracold atoms near a Feshbach resonance [105] has also been discussed. When the system is totally polarized, it has been observed to behave like a weakly interacting Fermi gas [106. Here, we wish to explore to which extent and up to which densities we are in agreement with such conclusions, and how this and other observations depend on the chiral order and the resolution scale.

In contrast with previous calculations, our recent work summarized here contains the following novelties:

- We consider both cutoff dependence and truncation error for the purpose of uncertainty quantification of chiral EFT. Although incomplete in the $3 \mathrm{NF}$ at $\mathrm{N}^{3} \mathrm{LO}$, our calculations are a substantial step in that direction. We note, further, that the contribution from the $3 \mathrm{NF}$ at $\mathrm{N}^{3} \mathrm{LO}$ was found to be very small in neutron matter for the potentials in our perview [86], about $-0.5 \mathrm{MeV}$ at normal density. Here, we consider neutron matter or highly neutron-rich matter.

- For the first time, we present results for both spin and isospin asymmetries within the framework of chiral forces. These tools are necessary to assess, for instance, the sensitivity of the results (particularly, the potential onset of a phase transition) to the presence of a non-zero proton fraction.

For a detailed description of the formalism, the reader is referred to Ref. [109]. Here, we will just summarize some definitions which are necessary for the discussion which follows.

In a spin-polarized and isospin asymmetric system with fixed total density, $\rho$, the partial densities of each species are

$$
\rho_{n}=\rho_{n u}+\rho_{n d}, \quad \rho_{p}=\rho_{p u}+\rho_{p d}, \quad \rho=\rho_{n}+\rho_{p},
$$

where $u$ and $d$ refer to up and down spin-polarizations, respectively, of protons $(p)$ or neutrons $(n)$. The isospin and spin asymmetries, $\alpha, \beta_{n}$, and $\beta_{p}$, are defined in a natural way:

$$
\alpha=\frac{\rho_{n}-\rho_{p}}{\rho}, \quad \beta_{n}=\frac{\rho_{n u}-\rho_{n d}}{\rho_{n}}, \quad \beta_{p}=\frac{\rho_{p u}-\rho_{p d}}{\rho_{p}} .
$$

The density of each individual component can be related to the total density by

$$
\begin{aligned}
& \rho_{n u}=\left(1+\beta_{n}\right)(1+\alpha) \frac{\rho}{4}, \quad \rho_{n d}=\left(1-\beta_{n}\right)(1+\alpha) \frac{\rho}{4}, \\
& \rho_{p u}=\left(1+\beta_{p}\right)(1-\alpha) \frac{\rho}{4}, \quad \rho_{p d}=\left(1-\beta_{p}\right)(1-\alpha) \frac{\rho}{4},
\end{aligned}
$$

where each partial density is related to the corresponding Fermi momentum through $\rho_{\tau \sigma}=\left(k_{F}^{\tau \sigma}\right)^{3} /\left(6 \pi^{2}\right)$. The average Fermi momentum and the total density are related in the usual way as $\rho=\left(2 k_{F}^{3}\right) /\left(3 \pi^{2}\right)$. 


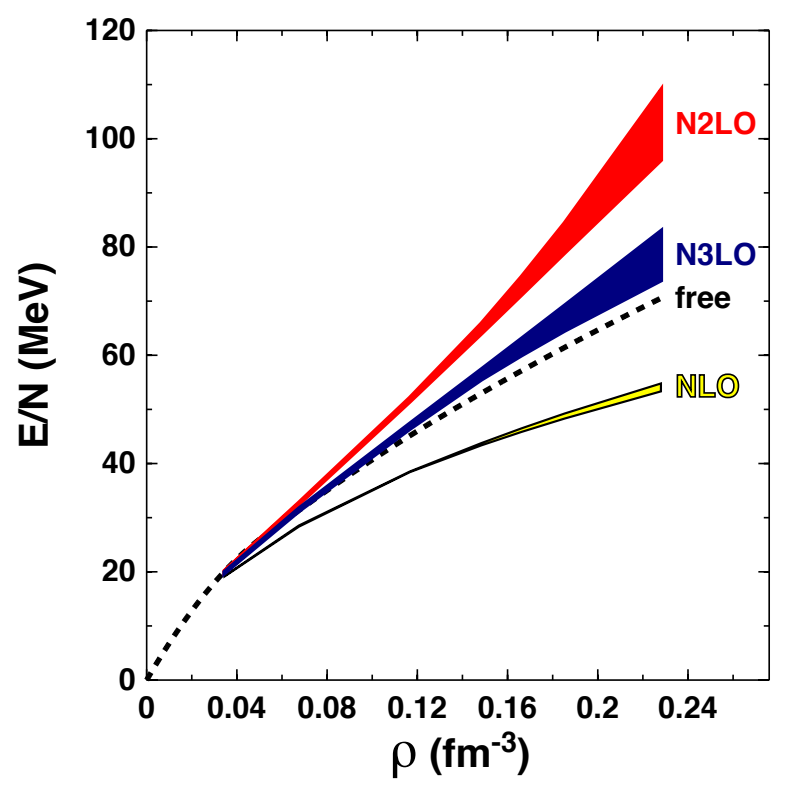

Figure 24. Energy per neutron in fully polarized neutron matter as a function of density. The yellow and red bands represent the uncertainities due to cutoff variations obtained in the complete calculations at $\mathrm{NLO}$ and $\mathrm{N}^{2} \mathrm{LO}$, respectively. The blue band is the result of the same cutoff variations applied to our exploratory $\mathrm{N}^{3} \mathrm{LO}$ calculation, see text for details. The dotted curve shows the energy of the free Fermi gas. (Figure reproduced from Ref. [109].)

We show in Fig. 24 the energy per particle in fully polarized neutron matter as a function of density. The yellow and red bands represent the predictions of complete calculations at second and third order, respectively, of chiral effective field theory, while the blue band shows the predictions obtained with the exploratory $\mathrm{N}^{3} \mathrm{LO}$ calculation as described above. For each band, the width is obtained by changing the cutoff between $450 \mathrm{MeV}$ and $600 \mathrm{MeV}$.

At $\mathrm{N}^{2} \mathrm{LO}$ and $\mathrm{N}^{3} \mathrm{LO}$, cutoff dependence is generally moderate up to saturation density. At NLO, the cutoff dependence is practically negligible throughout. In unpolarized neutron matter, on the other hand, the largest cutoff dependence was seen at NLO [66]. This suggests that, in unpolarized NM, the larger cutoff sensitivity at NLO is mostly due to singlet states, particularly ${ }^{1} S_{0}$, which are absent from the polarized system. At the same time, $3 \mathrm{NFs}$ do not appear at NLO, implying that most of the cutoff dependence in polarized $\mathrm{NM}$ at $\mathrm{N}^{2} \mathrm{LO}$ and $\mathrm{N}^{3} \mathrm{LO}$ is caused by the $3 \mathrm{NF}$ contributions.

Clearly, the variations associated with changing the cutoff are not a good indicator of the uncertainty at a given order of chiral effective field theory, as the results from one order to the other do not overlap. Furthermore, the predictions do not show a good convergence pattern, although some indication of slow convergence can be seen when moving from $\mathrm{N}^{2} \mathrm{LO}$ to our $\mathrm{N}^{3} \mathrm{LO}$ calculation.

As can be concluded from Table 7 , the predictions from the $\mathrm{N}^{3} \mathrm{LO}$ calculation are close to the free Fermi gas energy, at least up to saturation densities. Our results with 


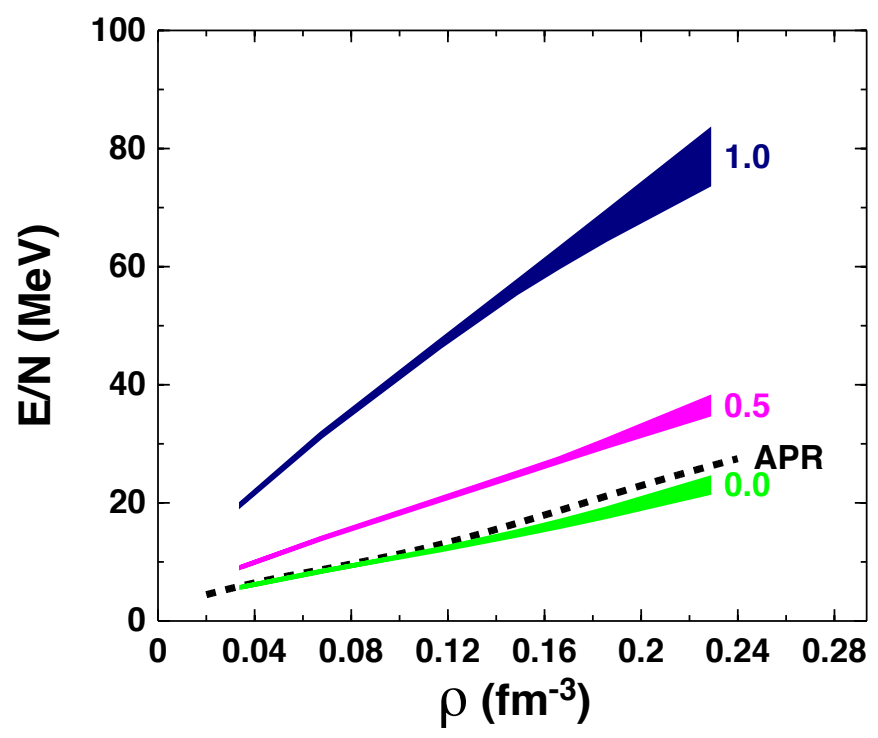

Figure 25. Energy per neutron in pure neutron matter as a function of density at $\mathrm{N}^{3} \mathrm{LO}$. From lowest to highest curve: unpolarized NM; partially polarized NM, with $\beta_{n}=0.5$; fully polarized NM $\left(\beta_{n}=1\right)$. The width of each band shows the uncertainty from varying the cutoff between 450 and $600 \mathrm{MeV}$. The black dotted line shows the predictions for the equation of state of unpolarized neutron matter from Ref. [107. (Figure reproduced from Ref. [109.)

the $\mathrm{N}^{3} \mathrm{LO}$ [28] $(\Lambda=500 \mathrm{MeV})$ potential are in good agreement with those from Ref. [106] using the same potential as well as three- and four-nucleon forces at $\mathrm{N}^{3} \mathrm{LO}$.

In Fig. 25, for our $\mathrm{N}^{3} \mathrm{LO}$ calculation, we compare predictions (along with their cutoff variations) of the energy per neutron in: unpolarized NM (green band), partially polarized NM (pink band), and fully polarized NM (blue band). For the partially polarized case, the value of $\beta_{n}$ is equal to 0.5 , corresponding to $75 \%$ of the neutrons being polarized in one direction and $25 \%$ in the opposite direction, see Eqs. (106). Clearly, a lesser degree of spin asymmetry (as compared to the ferromagnetic case) yields considerably less repulsion. There is definitely no sign of a phase transition, particularly to a ferromagnetic state, nor an indication that such transition may occurr at higher densities. This is consistent with what we observed earlier [98] with meson-theoretic interactions.

As a baseline comparison, we also include, for the unpolarized case, predictions based on a different approach, shown by the black dotted line in Fig. 25. These are taken from Ref. [107] and are based on the Argonne $v_{18}$ two-nucleon interaction plus the Urbana IX three body-force, using variational methods. The predictions are overall in reasonable agreement with our green band, although those from Ref. [107] show more repulsion as compared to the softer chiral interactions.

Most typically, models which do predict spin instability of neutron matter find the phase transition to occurr at densities a few times normal density. Such high densities are outside the domain of chiral perturbation theory. With some effective forces, though, it 


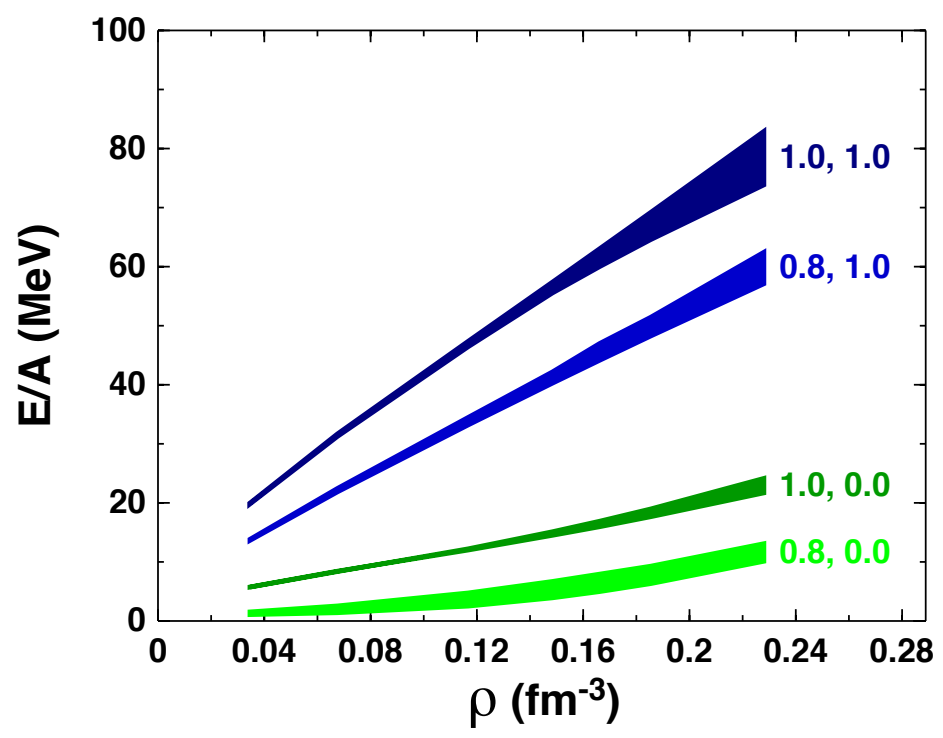

Figure 26. Energy per nucleon in neutron-rich matter as a function of density at $\mathrm{N}^{3} \mathrm{LO}$ and different conditions of isospin and spin polarization. The (brighter blue) band labeled as "0.8, 1.0" displays the results for neutron-rich matter with a proton fraction equal to $10 \%(\alpha=0.8)$ and fully polarized neutron $\left(\beta_{n}=1.0\right)$. The (brighter green) band labeled as "0.8, 0.0" refers to neutron-rich matter with the same proton fraction and no polarization $\left(\beta_{n}=0.0\right)$. The protons are unpolarized. For comparison, we also include the bands (darker blue and darker green) already shown in the previous figure, which refer to pure neutron matter $(\alpha=1)$ with fully polarized $\left(\beta_{n}=1\right)$ or unpolarized $\left(\beta_{n}=0\right)$ neutrons. The bands are obtained varying the cutoff between 450 and $600 \mathrm{MeV}$. (Figure reproduced from Ref. [109].)

was found [108] that a small fraction of protons can significantly reduce the onset of the threshold density for a phase transition to a spin-polarized state of neutron-rich matter. We explored this scenario by adding a small fraction of protons to fully polarized or unpolarized neutrons. From Eqs. 105)-(107), a proton fraction of $10 \%$ is obtained with $\alpha=0.8$. The results are displayed in Fig. 26, where a crossing of the bands labeled with " $0.8,1.0$ " and "0.8, 0.0", respectively, would indicate a phase transition. Thus we conclude that such transition is not predicted with chiral forces. By extrapolation, a transition to a polarized state would also appear very unlikely at higher densities.

To summarize this section, we have calculated the equation of state of (fully and partially) polarized neutron-rich matter. We performed complete calculations at second and third order of chiral effective field theory and calculations employing the $\mathrm{N}^{3} \mathrm{LO}$ $2 \mathrm{NF}$ plus the leading $3 \mathrm{NF}$. Results with both spin and isospin asymmetries have been presented for the first time with chiral forces in Refs. [109].

In all calculations, the cutoff dependence is moderate and definitely underestimates the uncertainty of each order. Concerning the latter, we do not see a satisfactory convergence pattern. The missing 3NFs are most likely not the main cause of uncertainty at $\mathrm{N}^{3} \mathrm{LO}$, since Ref. [106] has demonstrated that large cancelations take place between the $2 \pi$-exchange $3 \mathrm{NF}$ and the $\pi$-ring $3 \mathrm{NF}$ at $\mathrm{N}^{3} \mathrm{LO}$, while other $3 \mathrm{NF}$ contributions 


\begin{tabular}{|c||c|c|}
\hline Density $\left(\mathrm{fm}^{-3}\right)$ & $\Lambda(\mathrm{MeV})$ & $E_{F F G} / E$ \\
\hline 0.15 & 450 & 0.95 \\
& 500 & 0.92 \\
0.17 & 600 & 0.95 \\
& 450 & 0.95 \\
& 500 & 0.91 \\
& 600 & 0.93 \\
\hline
\end{tabular}

Table 7. Ratio of the energy per particle of a free Fermi gas to the energy per particle of polarized neutron matter around saturation density at $\mathrm{N}^{3} \mathrm{LO}$ (as described in the text) and for different values of the cutoff.

are very small (about $0.1-0.2 \mathrm{MeV}$ ). Clearly a calculation at $\mathrm{N}^{4} \mathrm{LO}$ is necessary to get a realistic indication of the EFT error at $\mathrm{N}^{3} \mathrm{LO}$. Such effort is in progress. If such calculation displays a reasonable convergence pattern, it will be strong evidence that polarized neutron matter, indeed, behaves nearly like a free Fermi gas, at least up to normal densities.

In our $\mathrm{N}^{3} \mathrm{LO}$ calculation, the energies of the unpolarized system at normal density are close to $16 \mathrm{MeV}$ for all cutoffs, whereas those in the polarized case are approximately $60 \mathrm{MeV}$. Thus, even in the presence of the large uncertainties discussed above, a phase transition to a ferromagnetic state can be excluded. This conclusion remains valid in the presence of a small proton fraction.

\subsection{Uncertainty analysis for predictions of the neutron skin in ${ }^{208} \mathrm{~Pb}$ at different orders of chiral effective field theory}

As mentioned at the beginning of Sec. 8, intense effort is going on to obtain reliable empirical information for the less known aspects of the EoS. Heavy-ion (HI) reactions are a popular way to seek constraints on the symmetry energy, through analyses of observables that are sensitive to the difference between the pressure in nuclear and neutron matter. Isospin diffusion data in HI collisions together with analyses based on isospin-dependent transport models, provide information on the slope of the symmetry energy. For a recent review on available constraints from a broad spectrum of experiments, see Ref. [110].

Concerning the lower densities, isospin-sensitive observables can also be identified among the properties of normal nuclei. The neutron skin of neutron-rich nuclei is a powerful isovector observable, being sensitive to the slope of the symmetry energy, which determines to which extent neutrons are pushed outwards to form the skin [111. Parityviolating electron scattering experiments are now a realistic option to determine neutron distributions with unprecedented accuracy. These experiments at low momentum transfer are especially suitable to probe neutron densities, because the $Z^{0}$ boson couples primarily to neutrons [112]. From the first electroweak observation of the neutron skin in a neutron-rich heavy nucleus, a values of $0.33_{-0.18}^{+0.16}$ for the neutron skin of ${ }^{208} \mathrm{~Pb}$ was 
determined [113], but the next PREX experiment aims to measure the skin within an uncertainty smaller by a factor of 3 (see Ref. [113] and references therein).

From the theoretical point of view, we stress once again that microscopic calculations with statistically meaningful uncertainties are essential to guide experiments. Therefore, following the spirit of Ref. [66], it is the purpose of this section to systematically examine and discuss predictions of the neutron skin in ${ }^{208} \mathrm{~Pb}$ at different orders of chiral EFT and changing resolution scale.

It is well established that the neutron skin thickness correlates with the derivative of the symmetry energy. The latter is often represented through the $L$ parameter,

$$
L=3 \rho_{0}\left(\frac{\partial E_{\text {sym }}(\rho)}{\partial \rho}\right)_{\rho_{0}} \approx 3 \rho_{0}\left(\frac{\partial e_{n . m .}(\rho)}{\partial \rho}\right)_{\rho_{0}},
$$

which originates from an expansion of the symmetry energy around the saturation point, $\rho_{0}$. The second (approximate) equality is due to the vanishing of the first derivative of the energy per particle in SNM at $\rho_{0}$, leaving a term proportional to the pressure in neutron matter. Nevertheless, $L$ depends sensitively on the saturation density, which can be quite different from model to model, particularly when considering different chiral orders and regulators. In other words, theoretical predictions of $L$ carry larger EFT uncertainties than the ones of just neutron matter pressure at some fixed density. To explore this point further, we will also compare predictions and uncertainties with those obtained using a phenomenological EoS for SNM consistent with the empirical saturation point.

8.3.1. Predictions with microscopic EoS for NM and SNM We calculate proton and neutron density distributions with a method described in an earlier work [114]. The method is based on an energy functional derived from the semi-empirical mass formula, where the volume and symmetry terms are contained in the isospin-asymmetric equation of state. Thus, we write the energy of a (spherical) nucleus as

$$
E(Z, A)=\int d^{3} r e(\rho, \alpha) \rho(r)+\int d^{3} r f_{0}\left(|\nabla \rho|^{2}+\beta\left|\nabla \rho_{I}\right|^{2}\right)+I_{C},
$$

where $I_{C}$ stands for the Coulomb term. In the above equation, $\rho$ and $\rho_{I}$ are the usual isoscalar and isovector densities, given by $\rho_{n}+\rho_{p}$ and $\left(\rho_{n}-\rho_{p}\right)$, respectively, $\alpha$ is the neutron asymmetry parameter, $\alpha=\rho_{I} / \rho$, and $e(\rho, \alpha)$ is the energy per particle in isospin-asymmetric nuclear matter. The constant $f_{0}$ in Eq. (109) is approximately 70 $\mathrm{MeV} \mathrm{fm}^{5}$, whereas the magnitude of $\beta$ is about $1 / 4$ [115]. (Even with variations of $\beta$ between -1 and +1 , we found that the contribution from that term was negligibly small, so we disregarded its contribution.)

The symmetry energy, $E_{\text {sym }}$, has been defined in Eq. (104). As discussed earlier, it is customary to retain only the term quadratic in $\alpha$ in Eq. (104).

The proton and neutron density functions are obtained by minimizing the value of the energy, Eq. (109), with respect to the paramaters of Thomas-Fermi distributions for proton and neutron densities. Although simple, this method has the advantage of allowing a very direct connection between the EoS and the properties of finite nuclei. 


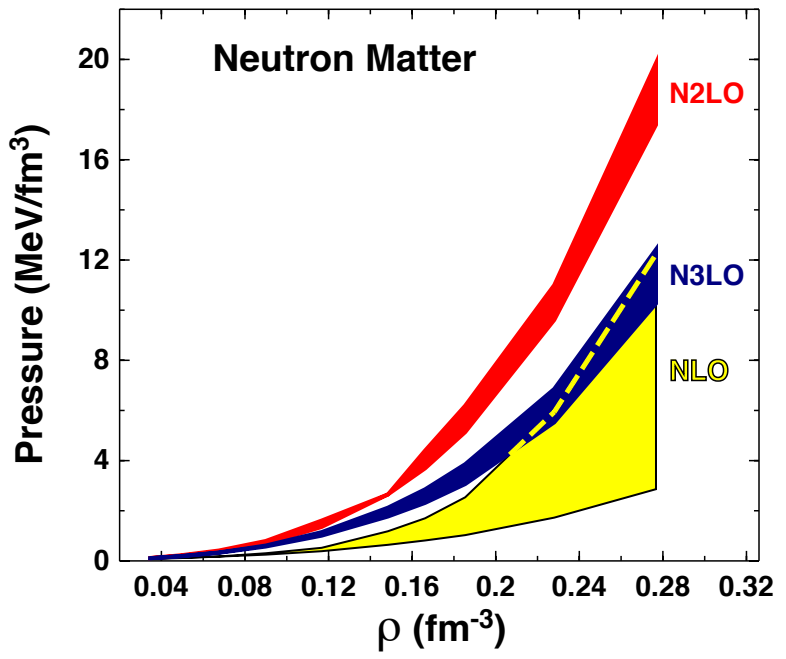

Figure 27. Pressure in pure neutron matter as a function of density, $\rho$. The yellow and red bands represent the uncertainties in the predictions due to cutoff variations as obtained in complete calculations at NLO and $\mathrm{N}^{2} \mathrm{LO}$, respectively. The blue band is the result of a calculation employing $\mathrm{N}^{3} \mathrm{LO} N N$ potentials together with $\mathrm{N}^{2} \mathrm{LO} 3 \mathrm{NFs}$. The dashed line shows the upper limit of the yellow band. (Figure reproduced from Ref. [111.)

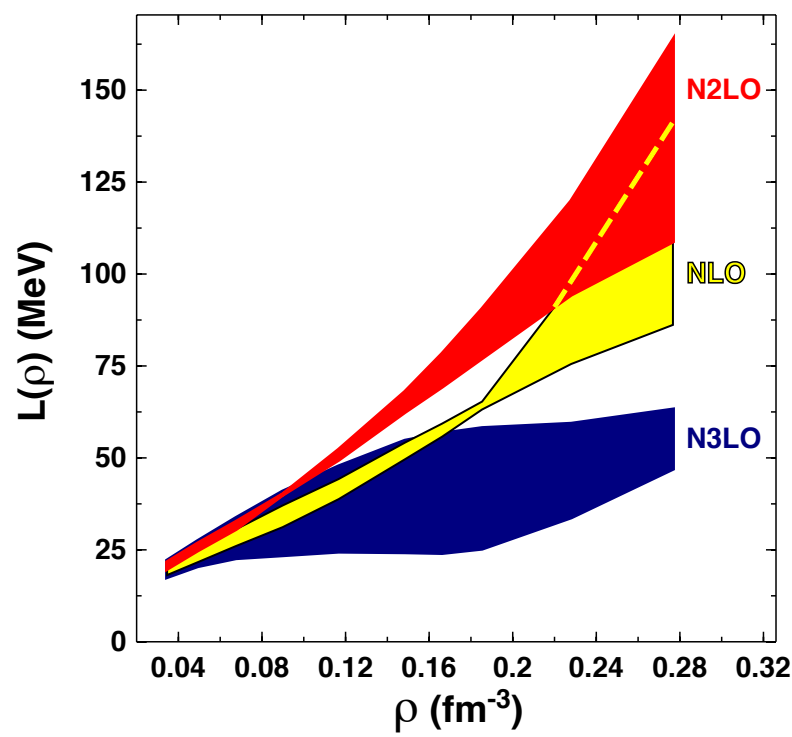

Figure 28. The $L$ parameter as a function of density, as defined in Eq. 1110). (Figure reproduced from Ref. [111.)

Furthermore, microscopic structure calculations for $A=208$ are presently not possible. In Ref. [114, our method was shown to yield realistic predictions for ${ }^{40} \mathrm{Ca},{ }^{90} \mathrm{Zr}$, and ${ }^{208} \mathrm{~Pb}$ with some of the Bonn meson-exchange potentials [5].

In the figures which follow, the size of each band is obtained from variations of the cutoff between 450 and $600 \mathrm{MeV}$ in the regulator applied to the $2 \mathrm{NF}$ and the 


\begin{tabular}{|c||c|c|c|}
\hline Order & $S(\mathrm{fm})$ & $L\left(\rho_{0}\right)(\mathrm{MeV})$ & $\rho_{0}\left(\mathrm{fm}^{-3}\right)$ \\
\hline $\mathrm{N}^{2} \mathrm{LO}$ & $0.21_{-0.02}^{+0.04}$ & $77.4_{-16.2}^{+31.2}$ & $0.167_{-0.022}^{+0.043}$ \\
& & & \\
$\mathrm{~N}^{3} \mathrm{LO}$ & $0.17_{-0.01}^{+0.02}$ & $39.9_{-15.7}^{+17.2}$ & $0.144_{-0.032}^{+0.032}$ \\
\hline
\end{tabular}

Table 8. Neutron skin thickness, $S$, in ${ }^{208} \mathrm{~Pb}$ at the specified order of chiral EFT as explained in the text. The corresponding values of the $L$ parameter and the saturation density are given in the last two columns.

3NF. In Fig. 27, the pressure in neutron matter is shown. The yellow and red bands represent the uncertainties in the predictions due to cutoff variations as obtained in complete calculations at $\mathrm{NLO}$ and $\mathrm{N}^{2} \mathrm{LO}$, respectively. The blue band is the result of a calculation employing $\mathrm{N}^{3} \mathrm{LO} N N$ potentials together with $3 \mathrm{NFs}$ at $\mathrm{N}^{2} \mathrm{LO}$. The pressure is proportional to the slope of the various curves which make up the corresponding bands shown in Fig. 24. We observe moderate cutoff dependence except at NLO and a slow convergence tendency with increasing order.

As already pointed out, the $L$ parameter, defined as in Eq. (108), is sensitive to the characteristics of the equation of state of symmetric matter through $\rho_{0}$. The latter changes dramatically from order to order as well as with changing cutoff, which can be clearly seen from Fig. 21. In Fig. 28, we show the $L$ parameter as a function of density, i. e.

$$
L(\rho)=3 \rho\left(\frac{\partial E_{\text {sym }}\left(\rho^{\prime}\right)}{\partial \rho^{\prime}}\right)_{\rho^{\prime}=\rho}
$$

which reflects the difference between the pressures in NM and in SNM at each density. The derivative of the EoS of SNM comes in through the symmetry energy and determines larger uncertainties than those seen in Fig. 27.

The predictions for the skin thickness of ${ }^{208} \mathrm{~Pb}$ are summarized in Table 8, along with the corresponding values of the $L$ parameter at the appropriate saturation density, different in each case and also reported in Table 8. Note that we do not show predictions at NLO because, at this low order, only the EoS with the largest cutoff (of $600 \mathrm{MeV}$ ) displays some (late) saturating behavior, cf. Fig. 21. The upper and lower errors are the distances of the largest and smallest values (when changing the cutoff) from the average.

The truncation error at order $\nu$ of chiral EFT is the difference between the predictions at orders $\nu+1$ and $\nu$. Thus, from the Table, we can estimate this error at $\mathrm{N}^{2} \mathrm{LO}$ to be about $0.04 \mathrm{fm}$. A similar estimate at $\mathrm{N}^{3} \mathrm{LO}$ would require knowledge of the prediction at $\mathrm{N}^{4} \mathrm{LO}$, which is not available. Assuming a (pessimistic) truncation error at $\mathrm{N}^{3} \mathrm{LO}$ of similar size as the one at $\mathrm{N}^{2} \mathrm{LO}$, we then summarize our predictions for the skin as $0.17 \pm 0.04 \mathrm{fm}$, where the error is likely to be smaller assuming a reasonable convergence rate. [In fact, if one takes the cutoff variation as a realistic estimate of the error (as it is approximately the case at $\mathrm{N}^{2} \mathrm{LO}$, cf. Table 8), then our $\mathrm{N}^{3} \mathrm{LO}$ prediction carries an error of $0.02 \mathrm{fm}$. 


\begin{tabular}{|c||c|c|}
\hline Order & $S(\mathrm{fm})$ & $L\left(\rho_{0}\right)(\mathrm{MeV})$ \\
\hline $\mathrm{NLO}$ & $0.126_{-0.003}^{+0.004}$ & $20.4_{-6.3}^{+8.8}$ \\
& & \\
$\mathrm{~N}^{2} \mathrm{LO}$ & $0.20_{-0.01}^{+0.01}$ & $70.6_{-8.0}^{+4.1}$ \\
& & \\
$\mathrm{~N}^{3} \mathrm{LO}$ & $0.172_{-0.005}^{+0.002}$ & $44.9_{-5.4}^{+3.8}$ \\
\hline
\end{tabular}

Table 9. As Table 8, but employing a phenomenological model for the EoS of SNM. See text for details.

\subsubsection{Using a phenomenological EoS for symmetric nuclear matter The nearly} linear correlation between skins and neutron matter pressure typically observed in phenomenological investigations of skins [116, 117] refers to a family of models with the same, or very similar, SNM properties which differ mostly in the slope of neutron matter. This scenario can be simulated, for instance, by combining an empirical SNM equation of state together with different (microscopic) NM EoS, thus separating out the role of neutron matter pressure and removing any model dependence originating from the details of the saturation point.

We repeated the calculations adopting, this time, the empirical EoS from Ref. [118] for SNM. The latter is obtained from a Skyrme-type energy density functional and has a realistic saturation point at $\rho_{0}=0.16 \mathrm{fm}^{-3}$ with energy per particle equal to $-16.0 \mathrm{MeV}$. The corresponding findings are displayed in Table 9. For this test, we also show the results at NLO, since the saturation point can be defined for all cases. Although the midvalues are reasonably consistent with those in Table 8, the uncertainties are much smaller, particularly for the $L$ parameter, as to be expected based on the previous observations. The much smaller uncertainty at $\mathrm{N}^{3} \mathrm{LO}$ reflects the negligible cutoff dependence of neutron matter pressure at that order, see Fig. 27.

With similar considerations as above with respect to the truncation error, we define the uncertainty at $\mathrm{N}^{2} \mathrm{LO}$ as the difference between the prediction at this order and the one at the next order, which gives approximately 0.03. Assuming a similar uncertainty at $\mathrm{N}^{3} \mathrm{LO}$, we estimate the skin thickness at $\mathrm{N}^{3} \mathrm{LO}$, when adopting an empirical parametrization for the EoS of SNM, to be $0.17 \pm 0.03$. We note, again, that this reflects the uncertainty in pure neutron matter at the low densities probed by the skin. Such uncertainty is small, consistent with the low-density behavior seen in Fig. 27.

We observe that our final estimate is consistent with the value reported in Ref. [119], where the skin is obtained through correlations from Ref. [117], and including a study based on the liquid drop model. This strengthens our confidence in the method we adopt to obtain the skin.

To summarize, the neutron skin is an important isospin-sensitive "observable", essentially determined by the difference in pressure between symmetric and neutron matter. We calculated the neutron skin of ${ }^{208} \mathrm{~Pb}$ with two- and three-body chiral interactions. The neutron and proton density functions are obtained in a simple 
approach based on the semi-empirical mass formula. We observed that, in fully microscopic calculations, model dependence from the details of SNM at the saturation point does impact predictions of the symmetry pressure and, to a lesser extent, the neutron skin.

At the low densities typically probed by studies of the skin, EFT theoretical uncertainties for the skin are small on a scale set by a realistic experimental uncertainty, particularly at the higher orders of chiral effective field theory.

Calculations at $\mathrm{N}^{4} \mathrm{LO}$ are needed for a better quantification of the truncation error at $\mathrm{N}^{3} \mathrm{LO}$, and thus a reliable comparison of the EFT error with the target uncertainty set by future PREX experiments. Concerning the latter, from Ref. [113] we learn that the target uncertainty of PREX II is a factor of 3 smaller than the one from the first PREX experiment, thus approximately \pm 0.05 . If accomplished, this will allow to discriminate between theoretical predictions, along with the measured central value. For instance, the present EFT predictions would not be consistent with a measurement such as 0.33 (the current central value) \pm 0.05 .

\section{Conclusions}

The past 20 years have seen great progress in our understanding of nuclear forces in terms of low-energy QCD. Key to this development was the realization that low-energy QCD is equivalent to an effective field theory (EFT) which has become known as chiral perturbation theory (ChPT). In this framework, two- and many-body forces emerge on an equal footing and the empirical fact that nuclear many-body forces are substantially weaker than the two-nucleon force is explained naturally.

We presented the current status of the development of chiral nuclear forces and discussed open questions and future challenges. We also reviewed some representative examples for typical applications of chiral forces in many-body systems. For this we chose, specifically, nuclear and neutron-rich matter, including isospin and spin asymmetries, as well as an analysis of neutron skin thickness predictions in a neutronrich nucleus.

Chiral forces have also been applied in ab initio calculations of finite nuclei (structure and reactions). Because of lack of space, we could not discuss this topic in this review and, therefore, we like to refer the interested reader to the comprehensive literature [64, 172, 173, 120, 121, 122, 123, 124, 125, 126, 127].

The importance of error quantification has finally been recognized in theoretical nuclear physics. We explored various sources of uncertainty systematically and noticed that the largest uncertainty comes from the truncation error of the chiral expansion (as given by the difference between the predictions at two consecutive orders). We also found that the predictions up to $\mathrm{N}^{3} \mathrm{LO}$ (fourth order) for many-body observables carry a truncation error that is, in general, substantially larger than the error of the empirical information, rendering the predictions inconclusive. Thus, in many applications of chiral EFT it may be necessary to proceed beyond fourth order. In any case, the convergence 
of the chiral expansion is one of the most important issues to which more work needs to be devoted in the near future.

\section{Acknowledgments}

The authors acknowledge support by the US Department of Energy under Grant No. DE-FG02-03ER41270. We thank our collaborators L. Coraggio, D. R. Entem, J. W. Holt, N. Itaco, N. Kaiser, L. E. Marcucci, and Y. Nosyk for their contributions to the work reviewed in this article.

\section{References}

[1] Reid R V 1968 Ann. Phys. (N.Y.) 50411

[2] Bryan R A and Scott B L 1964 Phys. Rev. 135 B434 Bryan R A and Scott B L 1969 Phys. Rev. 1771435

[3] Erkelenz K 1974 Phys. Rep. 13C 191

[4] Holinde K and Machleidt R 1975 Nucl. Phys. A 247495

Holinde K and Machleidt R 1976 Nucl. Phys. A 256479

[5] Machleidt R 1989 Adv. Nucl. Phys. 19189

[6] Lacombe M, Loiseau B, Richard J M, Vinh Mau R, Côté J, Pires P and de Tourreil R 1980 Phys. Rev. C 21861

[7] Machleidt R, Holinde K and Elster Ch 1987 Phys. Rep. 1491

[8] Weinberg S 1979 Physica 96A 327

[9] Weinberg S 1991 Nucl. Phys. B 3633

[10] Weinberg S 1992 Phys. Lett. B 295114

[11] Machleidt R and Entem D R 2011 Phys. Rep. 5031

[12] Epelbaum E, Hammer H-W and Meißner U-G 2009 Rev. Mod. Phys. 811773

[13] Meißner U-G 2016 Phys. Scr. 91033005

[14] Machleidt R 2014 Scholarpedia 9(1) 30710

[15] Machleidt R 2016 Symmetry 826

[16] Orginos K, Parreño A, Savage M J, Beane S R, Chang E and Detmold W 2015 Phys. Rev. D 92 114512

[17] Hatsuda T 2012 J. Phys. Conf. Ser. 381012020

[18] Scherer S 2003 Adv. Nucl. Phys. 27277

[19] Olive K A et al (Particle Data Group) 2014 Chin. Phys. C 380900001

[20] Coleman S, Wess J and Zumino B 1969 Phys. Rev. 1772239

Callan C G, Coleman S, Wess J and Zumino B 1969 Phys. Rev. 1772247

[21] Fettes N, Meißner U-G, Mojžiš M and Steininger S 2000 Ann. Phys. (N.Y.) 283273

Fettes N, Meißner U-G, Mojžiš M and Steininger S 2001 Ann. Phys. (N.Y.) 288249

[22] Krebs H, Gasparyan A and Epelbaum E 2012 Phys. Rev. C 85054006

[23] Ordóñez C, Ray L and van Kolck U 1996 Phys. Rev. C 532086

[24] van Kolck U 1994 Phys. Rev. C 492932

[25] Epelbaum E, Nogga A, Glöckle W, Kamada H, Meißner U-G and Witala H 2002 Phys. Rev. C 66064001

[26] Kaiser N 2000 Phys. Rev. C 61014003

[27] Kaiser N 2000 Phys. Rev. C 62024001

[28] Entem D R and Machleidt R 2003 Phys. Rev. C 68041001

[29] Entem D R, Kaiser N, Machleidt R and Nosyk Y 2015 Phys. Rev. C 91014002

[30] Krebs H, Gasparyan A and Epelbaum E 2013 Phys. Rev. C 87054007 
[31] Girlanda L, Kievsky A and Viviani M 2011 Phys. Rev. C 84014001

[32] Entem D R, Kaiser N, Machleidt R and Nosyk Y 2015 Phys. Rev. C 92064001

[33] Entem D R and Machleidt R unpublished

[34] Liu J et al 2010 Phys. Rev. Lett. 105181803

[35] Pavan M M, Arndt R A, Strakovsky I I and Workman R L 2000 Physica Scripta T87 65

[36] Arndt R A, Strakovsky I I, Workman R L and Pavan M M 2000 Physica Scripta T87 62

[37] Kaiser N, Brockmann R and Weise W 1997 Nucl. Phys. A 625758

[38] Epelbaum E, Glöckle W and Meißner U-G 2004 Eur. Phys. J. A 19401

[39] Kaiser N 2001 Phys. Rev. C 64057001

[40] Kaiser N 2001 Phys. Rev. C 63044010

[41] Kaiser N 2002 Phys. Rev. C 65017001

[42] Erkelenz K, Alzetta R and Holinde K 1971 Nucl. Phys. A 176413

[43] Machleidt R 1993 Computational Nuclear Physics 2 - Nuclear Reactions ed K Langanke et al (New York: Springer) pp 1-29

[44] Machleidt R 2001 Phys. Rev. C 63024001

[45] Arndt R A, Briscoe W J, Strakovsky I I and Workman R L 2006 Phys. Rev. C 74045205

[46] Koch R 1986 Nucl. Phys. A 448707

[47] Stoks V G J, Klomp R A M, Rentmeester M C M and de Swart J J 1993 Phys. Rev. C 48792

[48] Arndt R A, Strakovsky I I and Workman R L 1999 SAID Partial-Wave Analysis Facility Data Analysis Center, The George Washington University, Solution SM99 (Summer 1999)

[49] Entem D R and Machleidt R 2002 Phys. Rev. C 66014002

[50] Briscoe W J, Strakovsky I I and Workman R L 2007 SAID Partial-Wave Analysis Facility Data Analysis Center, The George Washington University, Solution SP07 (Spring 2007)

[51] Epelbaum E, Glöckle W and Meißner U-G 1998 Nucl. Phys. A 637107

[52] Lepage G P 1997 How to Renormalize the Schrödinger Equation arXiv:nucl-th/9706029

[53] Marji E et al 2013 Phys. Rev. C 88054002

[54] Entem D R and Machleidt R 2002 Phys. Lett. B 52493

[55] Stoks V G J, Klomp R A M, Terheggen C P F and de Swart J J 1994 Phys. Rev. C 492950

[56] Wiringa R B, Stoks V G J and Schiavilla R 1995 Phys. Rev. C 5138

[57] Epelbaum E, Glöckle W and Meißner U-G 2005 Nucl. Phys. A 747362

[58] Epelbaum E, Krebs H and Meißner U-G 2015 Eur. Phys. J. A 5153

[59] Epelbaum E, Krebs H and Meißner U-G 2015 Phys. Rev. Lett. 115122301

[60] Entem D R, Machleidt R and Witala H 2002 Phys. Rev. C 65064005

[61] Caurier E, Navratil P, Ormand W E and Vary J P 2002 Phys. Rev. C 66024314

[62] Coraggio L, Holt J W, Itaco N, Machleidt R, Marcucci L E and Sammarruca F 2014 Phys. Rev. C 89044321

[63] Ekstrőm et al 2013 Phys. Rev. Lett. 110192502

[64] Gezerlis A et al 2014 Phys. Rev. C 90054323

[65] Piarulli M, Girlanda L, Schiavilla R, Navarro Perez R, Amaro J E and Ruiz Arriola E 2015 Phys. Rev. C 91024003

[66] Sammarruca F, Coraggio L, Holt J W, Itaco N, Machleidt R and Marcucci L E 2015 Phys. Rev. C 91054311

[67] Fujita J-I and Miyazawa H 1957 Prog. Theor. Phys. 17360

[68] Coon S A, Scadron M D, McNamee P C, Barrett B R, Blatt D W E and McKeller B H J 1979 Nucl. Phys. A 317242

[69] Nogga A, Navratil P, Barrett B R and Vary J P 2006 Phys. Rev. C 73064002

[70] Navratil P, Gueorguiev V G, Vary J P, Ormand W E and Nogga A 2007 Phys. Rev. Lett. 99 042501

[71] Marcucci L E, Kievsky A, Rosati S, Schiavilla R and Viviani M 2012 Phys. Rev. Lett. 108052502

[72] Hagen G, Hjorth-Jensen M, Jansen G R, Machleidt R and Papenbrock T 2012 Phys. Rev. Lett. 108242501 
[73] Hagen G, Hjorth-Jensen M, Jansen G R, Machleidt R and Papenbrock T 2012 Phys. Rev. Lett. 109032502

[74] Viviani M, Giarlanda L, Kievsky A and Marcucci L E 2013 Phys. Rev. Lett. 111172302

[75] Ishikawa S and Robilotta M R 2007 Phys. Rev. C 76014006

[76] Bernard V, Epelbaum E, Krebs H and Meißner U-G 2008 Phys. Rev. C 77064004

Bernard V, Epelbaum E, Krebs H and Meißner U-G 2011 Phys. Rev. C 84054001

[77] Skibinski R et al 2011 Phys. Rev. C 84054005

[78] Witala H et al 2013 Few-Body Syst. 54897

[79] Tews I, Krüger T, Hebeler K and Schwenk A 2013 Phys. Rev. Lett. 110032504

[80] Epelbaum E 2007 Eur. Phys. J. A 34197

[81] Rozpedzik D, Golak J, Skibinski R, Witala H, Glöckle W, Epelbaum E, Nogga A and Kamada H 2006 Acta Phys. Polon. B 372889 (arXiv:nucl-th/0606017)

[82] Kaiser N 2012 Eur. Phys. J. A 48135

[83] Kaiser N and Milkus R 2016 Eur. Phys. J. A 524

[84] Furnstahl R J, Phillips D R and Wesolowski S 2015 J. Phys. G 42034028

[85] Coraggio L, Holt J W, Itaco N, Machleidt R and Sammarruca F 2013 Phys. Rev. C 87014322

[86] Krüger T, Tews I, Hebeler K and Schwenk A 2013 Phys. Rev. C 88025802

[87] Bogner S K, Schwenk A, Furnstahl R J and Nogga A 2005 Nucl. Phys. A 76359

[88] Hebeler K, Bogner S K, Furnstahl R J, Nogga A and Schwenk A 2011 Phys. Rev. C 83031301

[89] Gezerlis A, Tews I, Epelbaum E, Gandolfi S, Hebeler K, Nogga A and Schwenk A 2013 Phys. Rev. Lett. 111032501

[90] Baardsen G et al 2013 Phys. Rev. C 88054312

[91] Hagen G et al 2014 Phys. Rev. C 89014319

[92] Navarro Perez R, Garrido E, Amaro J E and Ruiz Arriola E 2014 Phys. Rev. C 90047001

[93] Alonso D and Sammarruca F 2003 Phys. Rev. C 67054301

[94] Drischler C, Soma V and Schwenk A 2014 Phys. Rev. C 89025806

[95] Kaiser N 2015 Phys. Rev. C 91065201

[96] Sammarruca F 2010 Phys. Rev. C 82027307

[97] Lane A M 1962 Nucl. Phys. 35676

[98] Sammarruca F 2011 Phys. Rev. C 83064304

[99] Sammarruca F and Krastev P 2007 Phys. Rev. C 75034315

[100] Vidaña I and Bombaci I 2002 Phys. Rev. C 66045801

[101] Isayev A A and Yang J 2004 Phys. Rev. C 70064310

[102] Marcos S, Niembro R, Quelle M L and Navarro J 1991 Phys. Lett. B 271277

[103] Carlson J, Chang S-Y, Pandharipande V R and Schmidt K E 2003 Phys. Rev. Lett. 91050401

[104] Carlson J, Gandolfi S and Gezerlis A 2012 PTEP 01A209

[105] Bloch I, Dalibard J and Zwerger W 2008 Rev. Mod. Phys. 80885

[106] Krüger T, Hebeler K and Schwenk A 2014 Phys. Lett. B 74418 and references therein

[107] Akmal A, Pandharipande V R and Ravenhall D J 1998 Phys. Rev. C 591804

[108] Isayev A A and Yang J 2004 Phys. Rev. C 69025801

[109] Sammarruca F, Machleidt R and Kaiser N 2015 Phys. Rev. C 92054327

[110] Tsang M B et al 2012 Phys. Rev. C 86015803 and references therein

[111] Sammarruca F 2015 Symmetry 71646 and references therein

[112] Horowitz C J, Pollock S J, Souder P A and Michaels R 2001 Phys. Rev. C 63025501

[113] Abrahamyan S et al (PREX Collaboration) 2012 Phys. Rev. Lett. 108112502

[114] Alonso D and Sammarruca F 2003 Phys. Rev. C 68054305

[115] Furnstahl R J 2002 Nucl. Phys. A 70685

[116] Typel S and Brown B A 2001 Phys. Rev. C 64027302

[117] Brown B A 2000 Phys. Rev. Lett. 855296

[118] Alam N, Agrawal B K, De J N, Samaddar S K and Colò G 2014 Phys. Rev. C 90054317

[119] Hebeler K and Schwenk A 2014 Eur. Phys. J. A 5014011 
[120] Binder S, Langhammer J, Calci A and Roth R 2014 Phys. Lett. B 736119

[121] Barrett B R, Navratil P and Vary J P 2013 Prog. Part. Nucl. Phys. 69131

[122] Hagen G, Papenbrock T, Hjorth-Jensen M and Dean D J 2014 Rept. Prog. Phys. 77096302

[123] Carlson J, Gandolfi S, Pederiva F, Pieper S C, Schiavilla R, Schmidt K E and Wiringa R B 2015 Rev Mod. Phys. 871067

[124] Ekstrőm A et al 2015 Phys. Rev. C 91051301

[125] Carlsson B D et al 2016 Phys. Rev. X 6011019

[126] Navrtil P, Quaglioni S, Hupin G, Romero-Redondo C and Calci A 2016 Phys. Scr. 91053002

[127] Hagen G, Hjorth-Jensen M, Jansen G R and Papenbrock T 2016 Phys. Scr. 91063006 